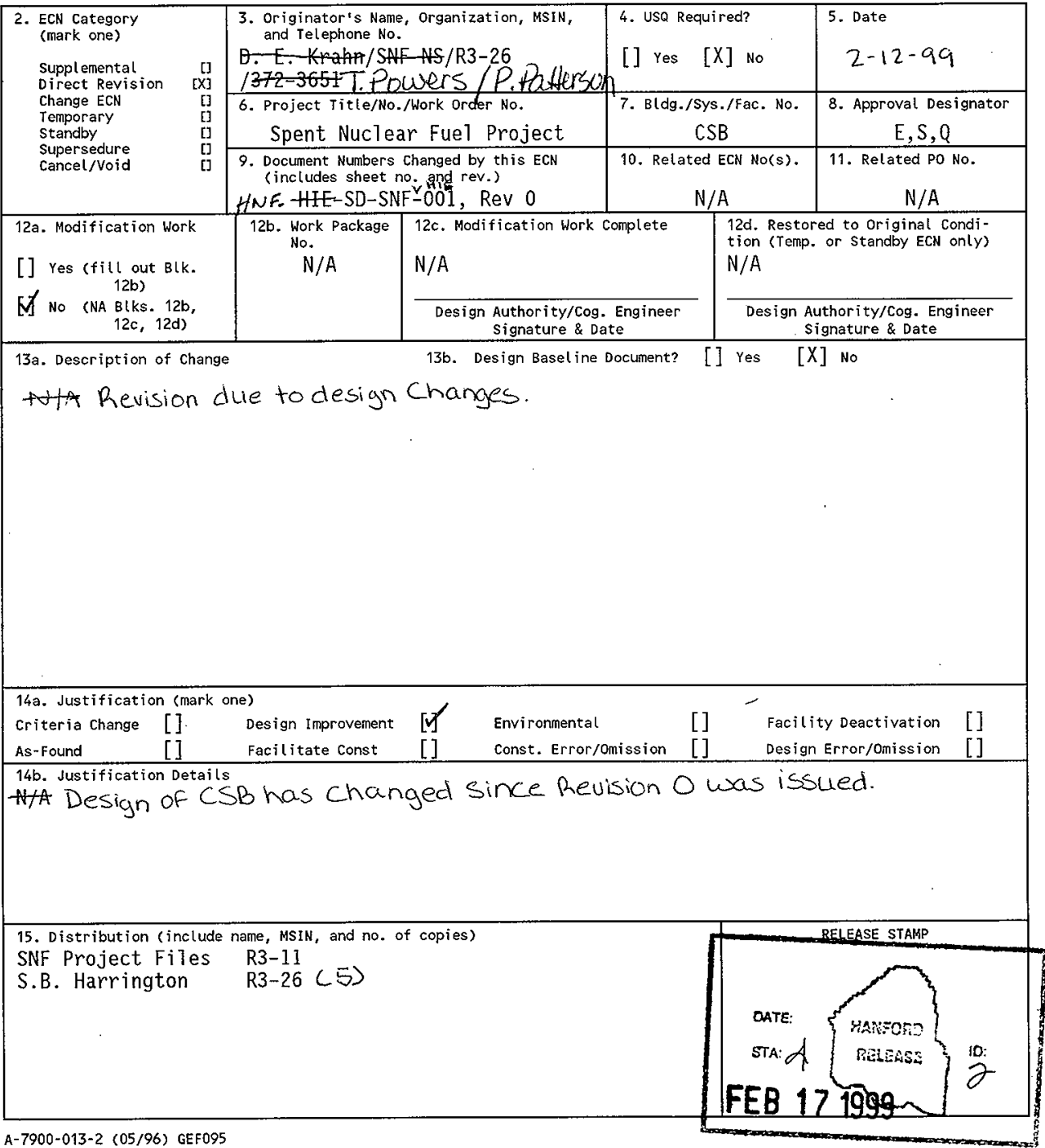




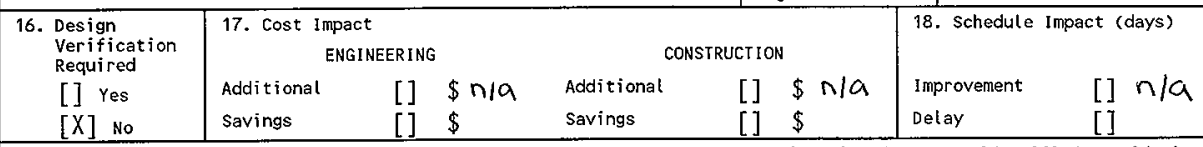

19. Change Impact Review: Indicate the related documents (other than the engineering documents identified on side 1) that will be affected by the change described in Block 13 . Enter the affected document number in $B$ lock 20.

SDD/DD
Functional Design Criteria
Operating Specification
Criticality Specification
Conceptual Design Report
Equipment Spec.
Const. Spec.
Procurement Spec.
Vendor Information
OM Manual
FSAR/SAR
Safety Equipment List
Radiation Work Permit
Environmental Impact Statement
Environmental Report

[] Seismic/Stress Analysis

[]

[]

[]

[]

[]

[]

[]

[]

$[1]$

[]

[]

[]

[]
Stress/Design Report

Interface Control Drawing

Calibration Procedure

Installation Procedure

Maintenance Procedure

Engineering Procedure

Operating Instruction

Operating Procedure

Operational Safety Requirement

IEFD Drawing

Cell Arrangement Drawing

Essential Material Specification

Fac. Proc. Samp. Schedule

Inspection Plan

Inventory Adjustment Request
[]

[]

[]

[]

[]

[]

[]

[]

[]

[]

[]

[]

[]

[]

[]
Tank Calibration Manua!

Health Physics Procedure

Spares Multiple Unit Listing

Test Procedures/Specification

Component Index

ASME Coded Item

Human Factor Consideration

Computer Software

Electric Circuit Schedule

ICRS Procedure

Process Control Manual/Plan

Process Flow Chart

Purchase Requisition

Tickler File

20. Other Affected Documents: (NOTE: Documents (isted below will not be revised by this ECN.) Signatures below indicate that the signing organization has been notified of other affected documents listed below.

Document Number/Revision Document Number/Revision

$+7 / 2$ HNF- 3553 AnNEXA, ReN. O- CSB FSAR
Document Number Revision
21. Approvals

signature

Design Authority G. D.
Cog. Eng. D_Erahp

Cog. Mgr. R. P. Omberg $R P C$ pubeng

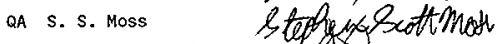

Safety L. J. Garvin

Environ. J. E. Turnbaugh

other

Cog Eng.t. Powers

P. Poutirson.

]
]
]
]
]
]
]
]
]
]
]
]
]
]




\section{Canister Storage Building Hazard Analysis Report}

\section{Tom B. Powers, * Paul R. Patterson}

Fluor Daniel Northwest, Inc., Richland, WA 99352

U.S. Department of Energy Contract DE-AC06-96RL13200

ECN: 647501

Org Code: 2F200

B\&R Code: 39EW40400
UC: 620

Charge Code: 105625/BB20

Total Pages: 120

Key Words: Hazard Analysis, Canister Storage Building

Abstract: This report describes the methodology used in conducting the Canister Storage Building (CSB) Hazard Analysis to support the final CSB Safety Analysis Report and documents the results.

* ARES Corporation

TRADEMARK DISCLAIMER. Reference herein to any specific commercial product, process, or service by trade name, trademark, manufacturer, or otherwise, does not necessarily constitute or imply its endorsement, recommendation, or favoring by the United States Government or any agency thereof or its contractors or subcontractors.

Printed in the United States of America. To obtain copies of this document, contact: Document Control Services, P.O. Box 950 , Mailstop H6-08, Richland WA 99352, Phone (509) 372-2420; Fax (509) 376-4989.
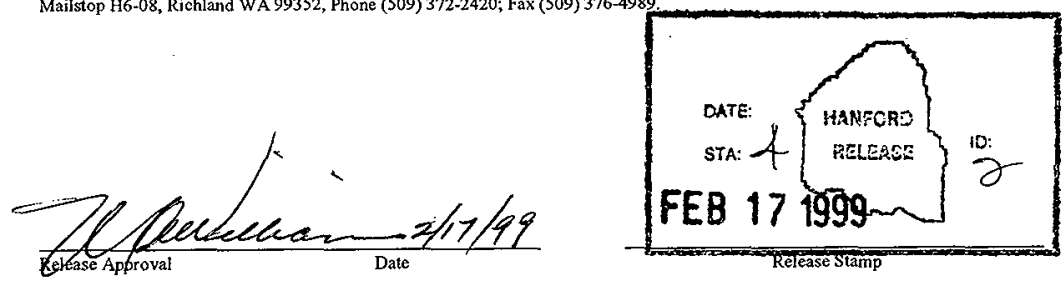

\section{Approved for Public Release}




\begin{tabular}{|l|c|c|}
\hline \multicolumn{1}{|c|}{ RECORD OF REVISION } & $\begin{array}{c}\text { (1) Document Number } \\
\text { HNF-SD-SNF-HIE-001, } \\
\text { Rev. 1 }\end{array}$ & Page 1 \\
\hline $\begin{array}{l}\text { (2) Title } \\
\text { Canister Storage Building Hazard Analysis Report }\end{array}$ & \\
\hline
\end{tabular}

\begin{tabular}{|c|c|c|c|}
\hline \multicolumn{4}{|c|}{ CHANGE CONTROL. RECORD } \\
\hline \multirow[t]{2}{*}{ (3) Revision } & \multirow[t]{2}{*}{ (4) Description of Change - Replace, Add, and Delete Pages } & \multicolumn{2}{|c|}{ Authorized for Release } \\
\hline & & (5) Cog. Engr. Date & (6) Cog. Mgr. \\
\hline & $(7)$ & & \\
\hline 0 & EDT: 607691 & & \\
\hline${ }^{l} R S$ & $\begin{array}{l}\text { ECN: } 647501 \\
\text { Revision due to design changes since the release of Revision } 0 .\end{array}$ & 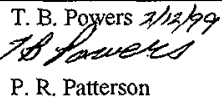 & 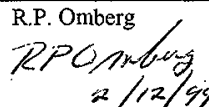 \\
\hline & & & \\
\hline & & & \\
\hline & & & \\
\hline & & & \\
\hline & & & \\
\hline
\end{tabular}


HNF-SD-SNF-HIE-001 REV 1

CANISTER STORAGE BUILDING

HAZARD ANALYSIS REPORT 
HNF-SD-SNF-HIE-001 REV 1

This page intentionally left blank. 
HNF-SD-SNF-HIE-001 REV 1

\section{CONTENTS}

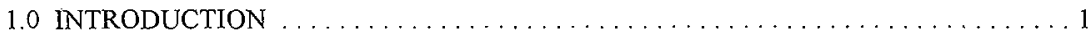

2.0 SCOPE OF THE HAZARD ANALYSIS $\ldots \ldots \ldots \ldots \ldots \ldots \ldots \ldots \ldots \ldots \ldots$

3.0 HAZARD ANALYSIS METHODOLOGY FOR NORMAL OPERATIONS $\ldots \ldots \ldots \ldots$

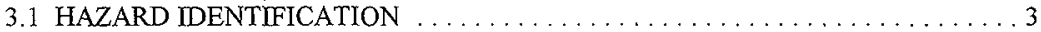

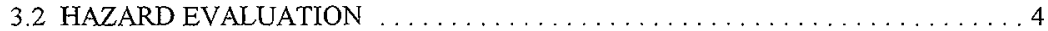

3.3 CANDIDATE ACCIDENT SELECTION $\ldots \ldots \ldots \ldots \ldots \ldots \ldots \ldots \ldots \ldots$

$3.4 \mathrm{HAZARD}$ ANALYSIS SUMMARY $\ldots \ldots \ldots \ldots \ldots \ldots \ldots \ldots$

4.0 HAZARD ANALYSIS FOR OFF-NORMAL MULTI-CANISTER OVERPACK

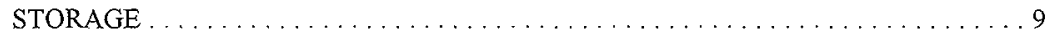

4.2 TECHNICAL SAFETY REQUIREMENTS COVERAGE . . . . . . . . . . 10

4.3 OFF-NORMAL MULTI-CANISTER OVERPACK STORAGE HAZARD

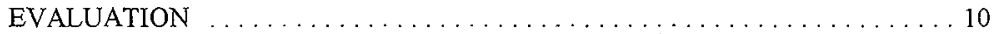

4.4 OFF-NORMAL MULTI-CANISTER OVERPACK STORAGE HAZARD BINS . . 10

4.5 OFF-NORMAL MULTI-CANISTER OVERPACK STORAGE HAZARD ANALYSIS SUMMARY $\ldots \ldots \ldots \ldots \ldots \ldots \ldots \ldots \ldots \ldots \ldots \ldots$

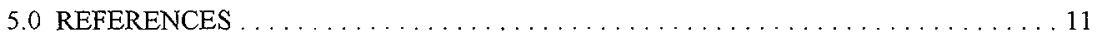

ATTACHMENTS

A CANISTER STORAGE BUILDING HAZARD ANALYSIS TEAM MEMBERS . . . . A-1 


\section{LIST OF TABLES}

1 Hazardous Material/Energy Source Checklist: Example $\ldots \ldots \ldots \ldots \ldots \ldots \ldots \ldots$

2 Hazardous Material/Energy Source Checklist: Truck Vestibule . . . . . . . . . . . 14

3 Hazardous Material/Energy Source Checklist: Service Area . . . . . . . . . . . . 15

4 Hazardous Material/Energy Source Checklist: Operating Area, Without Overpack,

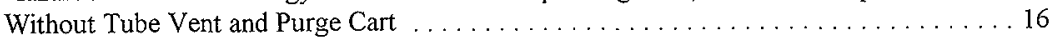

5 Hazardous Material/Energy Source Checklist: Sampling/Weld Station . . . . . . . . . 17

6 Hazardous Material/Energy Source Checklist: Vault $\ldots \ldots \ldots \ldots \ldots \ldots \ldots \ldots$

7 Hazardous Material/Energy Source Checklist: Support Building . . . . . . . . . . 19

8 Hazardous Material/Energy Source Checklist: Outside . . . . . . . . . . . . 20

9 Standard Industrial Hazards: Truck Vestibule $\ldots \ldots \ldots \ldots \ldots \ldots \ldots \ldots \ldots \ldots$

10 Standard Industrial Hazards: Service Area $\ldots \ldots \ldots \ldots \ldots \ldots \ldots \ldots \ldots \ldots$

11 Standard Industrial Hazards: Operating Area $\ldots \ldots \ldots \ldots \ldots \ldots \ldots \ldots \ldots \ldots$

12 Standard Industrial Hazards: Sampling/Weld Station $\ldots \ldots \ldots \ldots \ldots \ldots \ldots \ldots$

13 Standard Industrial Hazards: Support Building . . . . . . . . . . . . . . . . . . . 29

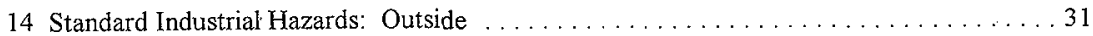

15 Canister Storage Building Material at Risk (Type, Form, and Quantity) . . . . . . 33

16 Canister Storage Building Hazard Analysis: Truck Vestibule . . . . . . . . . . 35

17 Canister Storage Building Hazard Analysis: Service Area . . . . . . . . . . . 43

18 Canister Storage Building Hazard Analysis: Operating Area $\ldots \ldots \ldots \ldots \ldots \ldots \ldots$

19 Canister Storage Building Hazard Analysis: Sampling/Weld Station . . . . . . . . . 64

20 Canister Storage Building Hazard Analysis: Vault $\ldots \ldots \ldots \ldots \ldots \ldots \ldots \ldots$

21 Canister Storage Building Hazard Analysis: Support Building $\ldots \ldots \ldots \ldots \ldots \ldots$ 


\section{LIST OF TABLES (Continued)}

22 Canister Storage Building Hazard Analysis: Outside $\ldots \ldots \ldots \ldots \ldots \ldots \ldots \ldots$

23 Release Characteristics for Hazardous Conditions Associated with Offsite (Site Boundary) and Onsite (Collocated Worker) Receptors . . . . . . . . . . 87

24 Binned Listing of Candidate Accidents . . . . . . . . . . . . . . . . . 94

25 Three-by-Three Likelihood and Consequence Ranking Matrix . . . . . . . . . . . 96

26 Hazardous Material/Energy Source Checklist: Off-Normal Multi-Canister

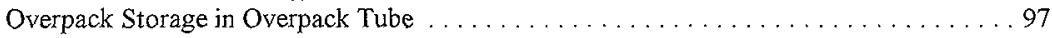

27 Canister Storage Building Hazard Analysis: Off-normal Multi-Canister Overpack Storage in Overpack Storage Tube . . . . . . . . . . . . . . . . . . 99

28 Binned Listing of Candidate Accidents for Off-normal Multi-Canister

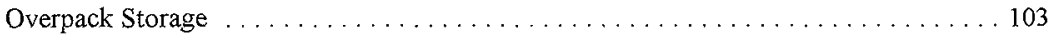




\section{HNF-SD-SNF-HIE-001 REV 1}

\section{LIST OF TERMS}

CSB Canister Storage Building

FSAR final safety analysis report

MCO multi-canister overpack

MHM multi-canister overpack handling machine 


\section{CANISTER STORAGE BUILDING HAZARD ANALYSIS REPORT}

\subsection{INTRODUCTION}

This report describes the methodology used in conducting the Canister Storage Building (CSB) hazard analysis to support the CSB final safety analysis report (FSAR) and documents the results. The hazard analysis was performed in accordance with the DOE-STD-3009-94, Preparation Guide for U.S. Department of Energy Nonreactor Nuclear Facility Safety Analysis Reports, and meets the intent of HNF-PRO-704, Hazard and Accident Analysis Process. This hazard analysis implements the requirements of DOE Order 5480.23, Nuclear Safety Analysis Reports.

\subsection{SCOPE OF THE HAZARD ANALYSIS}

The CSB hazard analysis covered normal, intended, CSB operations for handling and storing a sealed multi-canister overpack (MCO). Also identified and analyzed were the potential hazards associated with storing an off-normal MCO in an overpack storage tube following undetermined accident recovery actions. Chapter 3.0 of this report describes the hazard analysis for normal conditions, and Chapter 4.0 describes the hazard analysis for the off-normal MCO storage.

The hazard analysis was based on the design and operations described in Chapters A2.0 and A4.0 of HNF-3553, Spent Nuclear Fuel Project Final Safety Analysis Report, Annex A, "Canister Storage Building Final Safety Analysis Report." The analysis included review of a draft operation flow diagram and draft operating procedures. The following normal CSB operations were considered:

- Receiving the transporter containing the cask- $\mathrm{MCO}$ and moving it into the facility

- Moving the cask-MCO to the service area and removing the cask lid

- Transporting the MCO from the service area to the storage tube with the MCO handling machine (MHM)

- Transporting the MCO from the storage tube to the MCO sampling/weld station and returning it to the storage tube after sampling

- Conducting activities during MCO staging and interim storage. 
The following off-normal MCO storage operations also were considered.

- The event or accident leading to MCO damage has been terminated and recovery actions completed.

- The off-normal MCO is in place in the overpack storage tube.

- The overpack storage tube plug cover is installed.

- An inert atmosphere has been established in the overpack tube.

The following key sources of information were used to evaluate the hazards:

- HNF-3553, Spent Nuclear Fuel Project Final Safety Analysis Report, Annex A, "Canister Storage Building Final Safety Analysis Report"

- Chapters A2.0 and A4.0 for facility design and operations information

- Chapter A3.0 for the facility radioactive materials inventory

- Chapter A6.0 for evaluating the potential for hazards from nuclear criticality events

- WHC-SD-SNF-FHA-002, Fire Hazard Analysis for the Canister Storage Building; Ares Report 951107-001, Canister Storage Building Fire Code Equivalency Evaluation; Ares Report 951107-002, White Paper Presenting Recommended Approach to Fire Protection of the Operating Area of the Canister Storage Building; Letter 9655218, Project W-379, Spent Nuclear Fuel Canister Storage Building Request for Deviation from the United States Department of Energy Order 6430.1A - Automatic Sprinkler Protection Requirements (Williams 1996a); and Letter 9655233, Project W-379, Spent Nuclear Fuel Canister Storage Building Request for Exemption from the United States Department of Energy Order 5480.7A - Automatic Fire Suppression System Requirements (Williams 1996b) for fire protection information

- HNF-SD-TP-SARP-017, Safety Analysis Report for Packaging, Onsite, Multi-Canister Overpack Cask, for coverage of accidents involving the transporter and transportation cask and for definition of assumptions inherent in defining the transportation window

- HNF-SD-SNF-SARR-005, Multi-Canister Overpack Topical Report, for criteria and assumptions related to the MCO design

- Representatives from the design authority and from facility operations for details of design, operating modes, and procedures. 


\subsection{HAZARD ANALYSIS METHODOLOGY FOR NORMAL OPERATIONS}

This section presents the methodology used to perform the CSB hazard analysis for normal operations. The hazard identification process systematically and comprehensively identified hazards that can contribute to the uncontrolled release of radioactive or hazardous materials or that can threaten the safety of facility workers. The hazard evaluation process identified hazardous conditions, determined causes and preventive and mitigative features, and qualitatively estimated the frequency and consequences of occurrence. The hazard evaluation was performed by a team of cognizant CSB operations and design personnel, safety analysts familiar with the CSB, and technical experts in specialty areas. Attachment $A$ lists the members of the hazard analysis team and describes the background and experience of each.

Results of the hazard evaluations were used to select candidate accidents for quantitative analysis. Results of the hazard and accident analyses were then used to identify safety structures, systems, and components, technical safety requirements, and other controls required to protect the public, workers, and environment. The hazard and accident analysis results also supported determination of the final facility hazard classification.

\subsection{HAZARD IDENTIFICATION}

The hazard analysis identified hazards associated with CSB design and operations. Hazards were defined as material (referred to as material at risk) that could have a potentially adverse effect on people, the CSB facility, or the environment, and as energy sources that could contribute to uncontrolled release of radioactive or hazardous material or to injury of personnel.

Hazard identification for the CSB was based on examination of the facility and operations descriptions provided in Chapters A2.0 and A4.0 of HNF-3553, Annex A, on an operation flow diagram and operating procedures, and on discussions with design representatives from Fluor Daniel Incorporated. The facility was divided into seven areas:

1. Truck vestibule (TV)

2. Service area (SA)

3. Operating area, including overpack storage tubes and tube vent and purge cart (OA)

4. Sampling/weld station (WS)

5. Vault (VL)

6. Support building (SB)

7. Outside (OU).

A standardized checklist, Table 1 , was used to identify potentially hazardous materials and energy sources present in each of the seven areas. Tables 2 through 8 show the hazard identification results for each area. Each identified hazard was assigned a unique designator based 
on the checklists to allow for tracking. The hazard identification checklists were developed by a subgroup of the hazard analysis team and reviewed and accepted by the entire team.

\subsection{HAZARD EVALUATION}

The hazard evaluation was a structured and systematic examination of the CSB facility and its operations using standard industry (American Institute of Chemical Engineers) hazard evaluation techniques. The first step in the hazard evaluation, once the hazards had been identified, was to screen the potentially hazardous materials and energy sources for those that presented only standard industrial hazards. These hazards are defined in DOE-STD-3009-94 as those that "are routinely encountered in general industry and construction, and for which national consensus codes and/or standards (e.g., Occupational Safety and Health Administration, transportation safety) exist to guide safe design and operation without the need for special analysis to define safe design and/or operational parameters." Tables 9 through 14 list by facility area the standard industrial hazards that do not contribute to the uncontrolled release of radioactive or hazardous material. The standard industrial hazards listed are controlled through the implementation of institutional safety programs as described in the programmatic sections of HNF-3553, Annex A. The hazard analysis team agreed by consensus to the results of the screening for standard industrial hazard items.

Hazardous conditions not identified and sorted out as standard industrial hazards were further characterized and evaluated as the analysis process progressed. The material at risk associated with the hazardous conditions were identified and defined using the hazard identification checklists for each facility area (Table 2 through Table 8 ), reference documentation, and input from project design and operations personnel. A summary of the materials at risk, in terms of hazard type, form, quantity, and location, is included in this report as Table 15.

The next step was to evaluate each hazardous condition. The team met in facilitated sessions to perform this portion of the evaluation. Hazard analysis worksheets were designed to capture the required information for each hazard. The hazards were entered into the tables by their unique identifier for further tracking. Using the worksheets and the hazard summary as a guide, each hazardous condition was assessed to identify potential accidents, causes, frequencies, and consequences, and to determine a qualitative likelihood of occurrence of the initiating event and the resulting consequence. The assessment of likelihood and consequence for each hazardous condition was a collective, qualitative judgment made by the hazard analysis team. The assessment estimated the likelihoods and consequences of each hazardous condition scenario in two cases. The first case considered designed passive features only. The second case considered designed passive features as well as credited active features and administrative features.

The completed hazard analysis worksheets, included in this report as Tables 16 through 22 , show the results of the hazard evaluation as compiled by the hazard analysis team. The evaluation results are based on the hazard identification results, material-at-risk summaries, reviews of the systems designs and planned operations, existing safety documentation, and the experience of 


\section{HNF-SD-SNF-FIE-001 REV 1}

hazard analysis team members. Each column of the hazard analysis tables is explained below to aid in understanding the information contained therein.

Location/checklist entry. This column contains each hazard's unique identifier, which indicates the facility area, the hazard checklist category, and the specific hazard. For example, a designator of TV-F-01 would represent the truck vestibule (TV), a linear kinetic hazard (F) from a car, truck, or bus (0l).

Hazard energy source/material. This column further defines the specific hazard under consideration (e.g., a moving transporter).

Hazardous condition. This column describes the hazardous condition that the energy source or material represents (e.g., transporter collision).

Cause. This column identifies initiators of the potential accident (e.g., transporter collision with facility structure [the potential accident] could be caused by human error on the driver's part, by mechanical failure of the vehicle, or by misplaced equipment). Typical potential causes include equipment failures, operational errors, abnormal operating conditions, poor operating practices, and environmental conditions. The causes of a potential accident are identified to support a qualitative frequency evaluation.

Potential accident. This column identifies potential accidents that could result from the identified hazardous conditions (e.g., transporter collision with facility structures, systems, or components or with personnel).

Consequence. This column identifies the potential effects of the hazardous condition and potential accident in terms of radioactive or hazardous material releases, and impact to personnel and facility systems, structures, and components.

Credited prevention. This column lists preventive safety features present within the facility that are credited with reducing the frequency of the hazard or accident. The credited features listed in this column (both engineered and administrative) include only the controls the accident analyst required to be implemented to support the actual accident analysis. These preventive controls (along with the mitigative controls) are those controls necessary to meet evaluation guidelines.

Frequency code. Two evaluations of the likelihood of occurrence of the hazardous condition and potential accident are listed in the column labeled "Frequency Code." The first frequency code subcolumn ranks the hazard and accident frequency by considering the impact of any passive features (e.g., structures, barriers) listed in the table but not the impact of active features or planned controls (e.g., valves, shipping restrictions). The second frequency code subcolumn ranks the hazardous condition and potential accident frequency considering preventive controls, inchding passive controls. The assessment of likelihood was a collective, qualitative judgment made 
by the hazard analysis team. The likelihood assessments resulted in frequency rankings based on the initiating event frequencies and subsequent failures on a per year basis. The qualitative criteria for likelihood assessments are as follows.

F3 The hazardous condition based on the causes postulated is likely to occur during facility lifetime.

F2 The hazardous condition based on the causes postulated is foreseeable, but unlikely.

F1 The hazardous condition based on the causes postulated is perhaps possible, but extremely unlikely.

Fo

The hazardous condition based on the causes postulated is considered too improbable to warrant further consideration.

Credited mitigation. This column lists mitigative safety features present within the facility that are credited with reducing the consequence of the hazard. The credited features listed in this column (both engineered and administrative) include only the controls the accident analyst required to be implemented to support the actual accident analysis. These mitigative controls (along with the preventive controls) are those controls necessary to meet evaluation guidelines. In some cases a control may reduce both the frequency and the consequence of a hazard.

Consequence code. Two evaluations of the potential effects of the hazardous condition on the health and safety of people and on the environment are listed in the column labeled "Consequence Code.". The first consequence code subcolumn ranks the hazard and accident consequence by considering the impact of any passive features (e.g., structures, barriers) listed in the table but not the impact of active features or planned controls (e.g, valves, shipping restrictions). The second consequence code subcolumn ranks the hazardous condition and potential accident consequence with mitigative controls, including passive controls. The assessment of the consequence for each hazardous condition was a collective, qualitative judgment made by the hazard analysis team. The qualitative criteria for consequence assessments are as follows.

S3 On the basis of material at risk and causes postulated, there is sufficient material and release energy to affect a receptor at the nearest point of uncontrolled public access.

S2 On the basis of material at risk and causes postulated, there is sufficient material and energy to affect an onsite receptor (collocated worker) $100 \mathrm{~m}$ from the source of the release. 
S1 On the basis of material at risk and causes postulated, the release is confined to the facility and affects facility workers.

On the basis of material at risk and causes postulated, there is insufficient material released to affect facility workers.

The more severe consequence categories encompass the less severe consequence categories. For example, a hazardous condition assessed as having onsite consequences (S2) is also considered to have facility worker consequences (S1).

Defense-in-depth for worker safety features. This column contains any additional controls that will reduce the likelihood or consequences even further, but no specific credit is taken for them in the quantitative analysis.

\subsection{CANDIDATE ACCDENT SELECTION}

The hazardous conditions identified by the hazard evaluation have been used to select candidate accidents for a more detailed, quantitative analysis in the CSB FSAR (HNF-3553, Annex A). The general selection criteria used were consistent with DOE-STD-3009-94: "The range of accident scenarios analyzed in a SAR should be such that a complete set of bounding conditions to define the envelope of accident conditions to which the operation could be subjected are evaluated and documented."

The team used the four-step process described below to identify specific hazardous conditions that, together, represented the "complete set of bounding conditions" requiring further analysis. In summary, the process involved creating representative sets (or "bins") of hazardous conditions having similar release characteristics, similar initiators, and/or similar controls, and. identifying (using a ranking matrix) the hazardous condition that represented the most severe consequences and the highest risk in each bin. The highest ranking hazardous condition in each bin bounded the other hazardous conditions in the bin and, therefore, lead to candidate accidents needing further analysis. These hazardous conditions and candidate accidents represent the "complete set of bounding conditions" for the CSB accident analysis.

The following four-step process was used by the evaluation team to select the CSB bounding accidents:

1. Initial screening

2. Assignment of release attributes

3. Creation of hazardous material release bins

4. Selection of representative bounding hazardous conditions for each release attribute category. 
In order to capture and record the relational nature of the data developed in the four steps, the results have been organized into two tables, Table 23 and Table 24 . The following sections describe each step, and identify where in Table 23 or Table 24 the related information is located.

Initial Screening. All hazardous conditions with a frequency of F1 (extremely unlikely) or greater and unmitigated consequences assessed as $S 3$ (offsite consequences) or $S 2$ (collocated worker consequences) were chosen for consideration as representative accidents. These hazardous conditions are listed in Table 23, with their frequency and consequence rankings listed under the column entitled "Frequency/consequence codes.". There were no hazardous conditions assessed as $\mathrm{S} 1$ (facility worker consequences) involving radiological hazards requiring detailed accident analysis. The S1 hazardous conditions are addressed qualitatively in the CSB FSAR (HNF-3553, Annex A). Hazard conditions having no consequences (SO) were dropped from consideration.

Assignment of Release Attributes. Each hazardous condition was evaluated and described in terms of certain release attributes related to uncontrolled release of the material at risk. This description was assembled to ensure that at least one candidate accident was selected to represent each unique set of release conditions. The following hazardous material release attributes were used:

- Energy available to release the hazardous material (high, medium, or low) (Table 23, "Release energy" column)

- Release location (Table 23, "Designator" column)

- Release initiator (Table 23, "Hazardous condition and initiators" column).

Creation of Hazardous Material Release Bins. As the hazardous condition release attributes were identified, each hazardous condition was assigned to a bin category. Assignment to a bin category was based upon the potential accident release characteristics, initiators, and/or proposed mitigative or preventative controls. Table 23 lists the bin category assignment for each hazardous condition under the "Bin" column heading. The final step in creating the release attribute bins was to assemble hazardous conditions having the same bin category into a listing. This listing is the basis for Table 24, in which the hazardous conditions are grouped into their bin categories under the "Candidate accident" column.

\section{Selection of Representative Bounding Hazardous Conditions for each Release}

Attribute Category. Within each bin category, the most severe hazardous condition, considering consequences, and the highest risk accident were identified using the three-by-three likelihood and consequence ranking matrix described in DOE-STD-3009-94 (see Table 25). In Table 24 the bin category hazardous conditions are listed in descending order with the highest ranking hazardous condition at the top. ore than one condition may have been required to provide the necessary bounding conditions for a bin. Table 24 identifies the bounding condition, or when necessary, bounding conditions for each bin. 
Unique hazardous conditions were identified and selected as a part of the accident analysis process. However, the binning process described here provided the basis for identification and selection of those unique conditions. Briefly, at the completion of design basis accident analysis for each bin category, the results were compared with the other hazardous conditions in the original bin to ensure that no unique and unanalyzed conditions existed.

\subsection{HAZARD ANALYSIS SUMMARY}

The final list of candidate accidents includes all hazardous conditions with a frequency of F1 (extremely unlikely) or greater and whose unmitigated consequences were assessed as S3 (offsite consequences) or S2 (collocated worker consequences). Table 24 provides the final list of candidate accidents sorted first by risk ranking and then by release energy. The table also identifies the hazardous condition, or conditions, chosen as representative and bounding of all other conditions listed in the bin.

\subsection{HAZARD ANALYSIS FOR OFF-NORMAL MULTI-CANISTER OVERPACK STORAGE}

This section presents the methodology used to perform the CSB hazard analysis for offnormal MCO storage as a facility function. The off-normal MCO storage function requires analysis because a potential exists for MCO damage to occur during normal facility operations or during an accident. If an MCO were damaged, it would be declared off-normal and placed into an overpack storage tube. The hazard identification and evaluation process for off-normal MCO storage was essentially identical to the process described in Chapter 3.0; therefore, the process description is not repeated in this chapter. One difference of note, however, is in the hazardous condition frequency and consequences ranking, which is described in Section 4.3.

\subsection{OFF-NORMAL MULTI-CANISTER OVERPACK STORAGE HAZARD ANALYSIS SCOPE}

The scope of the analysis for off-normal MCO storage operations included the following conditions.

- The event or accident leading to MCO damage has been terminated and recovery actions completed.

- The off-normal MCO is in place in the overpack storage tube. 
- The overpack storage tube plug cover is installed.

- An inert atmosphere has been established in the overpack tube.

This scope is consistent with DOE-STD-3009-94, which does not require hazard analysis of accidents and recovery sequences, but does require analysis for anticipated facility operations.

\subsection{TECHNICAL SAFETY REQUIREMENTS COVERAGE}

Potentially hazardous materials and energy sources associated with the off-normal MCO storage function are identified in Table 26. The hazards associated with off-normal MCO storage for specific facility areas are listed in Table 27 and are identified by their unique designator.

\subsection{OFF-NORMAL MULTI-CANISTER OVERPACK STORAGE HAZARD EVALUATION}

Table 27 presents the results of the hazard evaluation for each of the identified hazards. Standard industrial hazards for this activity were assumed to be identical to those for normal facility operations because no new hazards were identified, and worker activities were similar to normal operations and performed in previously analyzed facility areas.

Frequency and consequence rankings for the off-normal MCO storage function involve two new considerations in addition to those described in Chapter 3.0. These considerations are incorporated into the ranking in Table 27 and are described as follows.

FR FR (frequency of recovery event) describes the undetermined likelihood of the "off-normal hazardous condition" developing following termination and recovery of the initial event. Therefore, the off-normal frequency ranking is a product of FR and the frequency of the initial event (e.g., F3, F2, F1).

SR SR (severity of the recovery event) describes the undetermined magnitude of the release caused by the unanalyzed condition of the damaged $\mathrm{MCO}$. Therefore, the off-normal consequence ranking is a product of $\mathrm{SR}$ and the consequence of the initial event (e.g., S3, S2, or S1).

\subsection{OFF-NORMAL MULTI-CANISTER OVERPACK STORAGE HAZARD BINS}

Table 28 lists the off-normal MCO storage hazardous conditions in bins relative to potential accident type and initiator. 
HNF-SD-SNF-HIE-001 REV 1

\subsection{OFF-NORMAL MULTI-CANISTER OVERPACK STORAGE HAZARD ANALYSIS SUMMARY}

System and equipment design changes have resulted from early hazard and accident analysis activities. Installation of these passive preventative features reduces the likelihood of $\mathrm{MCO}$ damage (i.e., drops leading to MCO cracks, which provide an open path to the atmosphere). These preventative design features, when introduced into the hazard analysis process for offnormal MCO storage, reduced the risk ranking to below requirements for further analysis. However, the information is retained in this report for historical reference.

\subsection{REFERENCES}

Ares Report 951107-001, 1996, Canister Storage Building Fire Code Equivalency Evaluation, Rev. 0, Ares Corporation, Richland, Washington.

Ares Report 951107-002, 1996, White Paper Presenting Recommended Approach to Fire Protection of the Operating Area of the Canister Storage Building, Rev. 0, Ares Corporation, Richland, Washington.

DOE Order 5480.23, Nuclear Safety Analysis Reports, U.S. Department of Energy, Washington, D.C.

DOE Standard 3009-94, Preparation Guide for U.S. Department of Energy Nonreactor Nuclear Facility Safety Analysis Reports, U.S. Department of Energy, Washington, D.C.

HNF-3553, 1999, Spent Nuclear Fuel Project Final Safely Analysis Report, Annex A, “Canister Storage Building Final Safety Analysis Report," Rev. 0, Fluor Daniel Hanford, Incorporated, Richland, Washington.

HNF-PRO-704, Hazard and Accident Analysis Process, Fluor Daniel Hanford, Incorporated, Richland, Washington.

HNF-SD-SNF-SARR-005, 1998, Multi-Canister Overpack Topical Report, Rev.1, Draft, Fluor Daniel Hanford, Incorporated, Richland, Washington.

HNF-SD-TP-SARP-017, 1997, Safety Analysis Report for Packaging, Onsite, Multi-Canister Overpack Cask, Rev. 0, Fluor Daniel Hanford, Incorporated, Richland, Washington.

WHC-SD-SNF-FHA-002, 1996, Phase 2 Fire Hazard Analysis for the Canister Storage Building, Rev. 1, Fluor Daniel Hanford, Incorporated, Richland, Washington. 
Williams, N. H., 1996a, Project W-379, Spent Nuclear Fuel Canister Storage Building Request for Deviation from the United States Department of Energy Order 6430.1A-Automatic Sprinkler Protection Requirements (Letter 9655218 to E. D. Sellers, U.S. Department of Energy, Richland Operations Office, November 14), Fluor Daniel Hanford, Incorporated, Richland, Washington.

Williams, N. H., 1996b, Project W-379, Spent Nuclear Fuel Canister Storage Building Request for Exemption from the U.S. Department of Energy Order 5480.7A - Automatic Fire Suppression System Requirements (Letter 9655233 to E. D. Sellers, U.S. Department of Energy, Richland Operations Office, November 14), Fluor Daniel Hanford, Incorporated, Richland, Washington. 
䓪

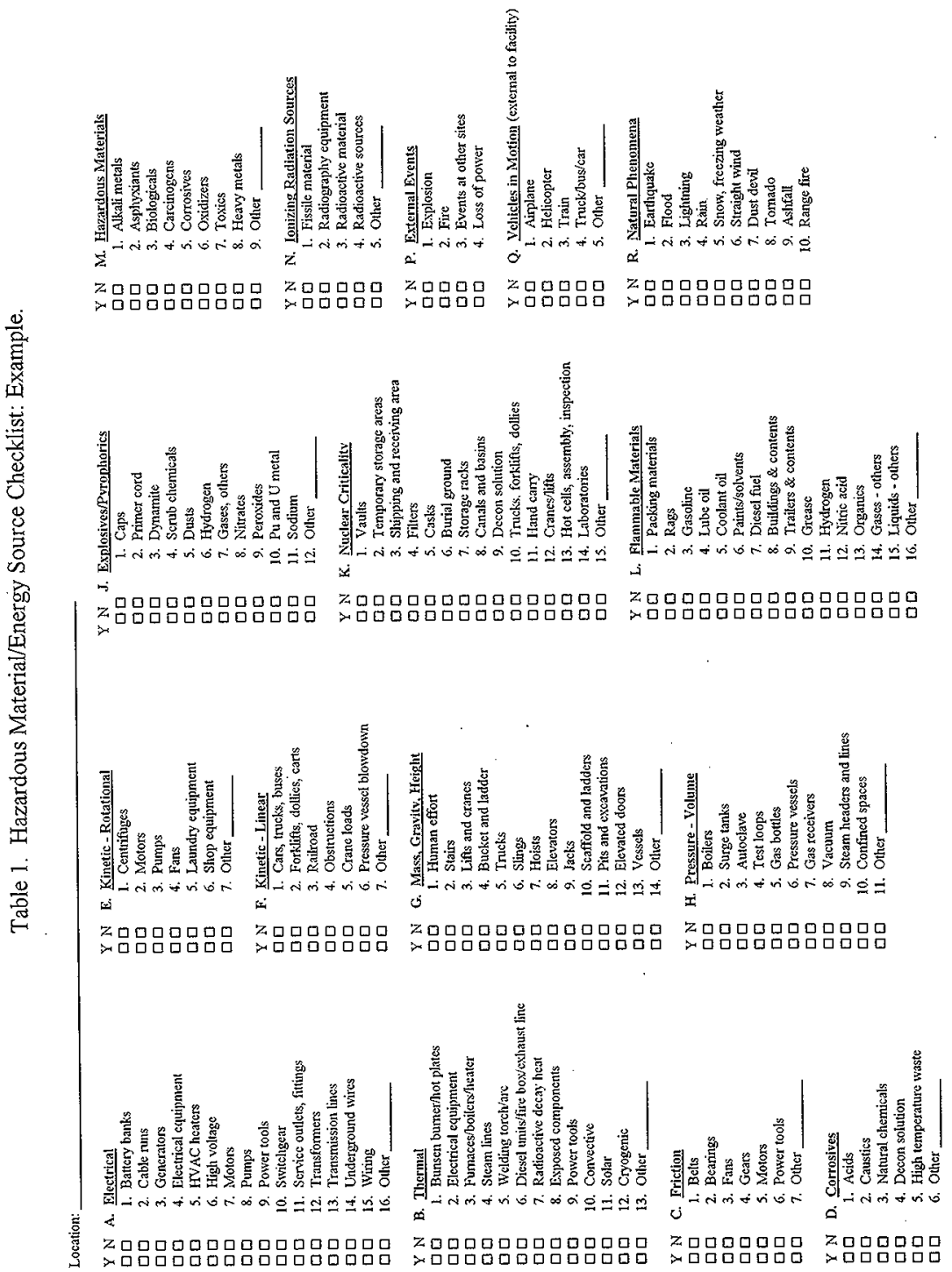



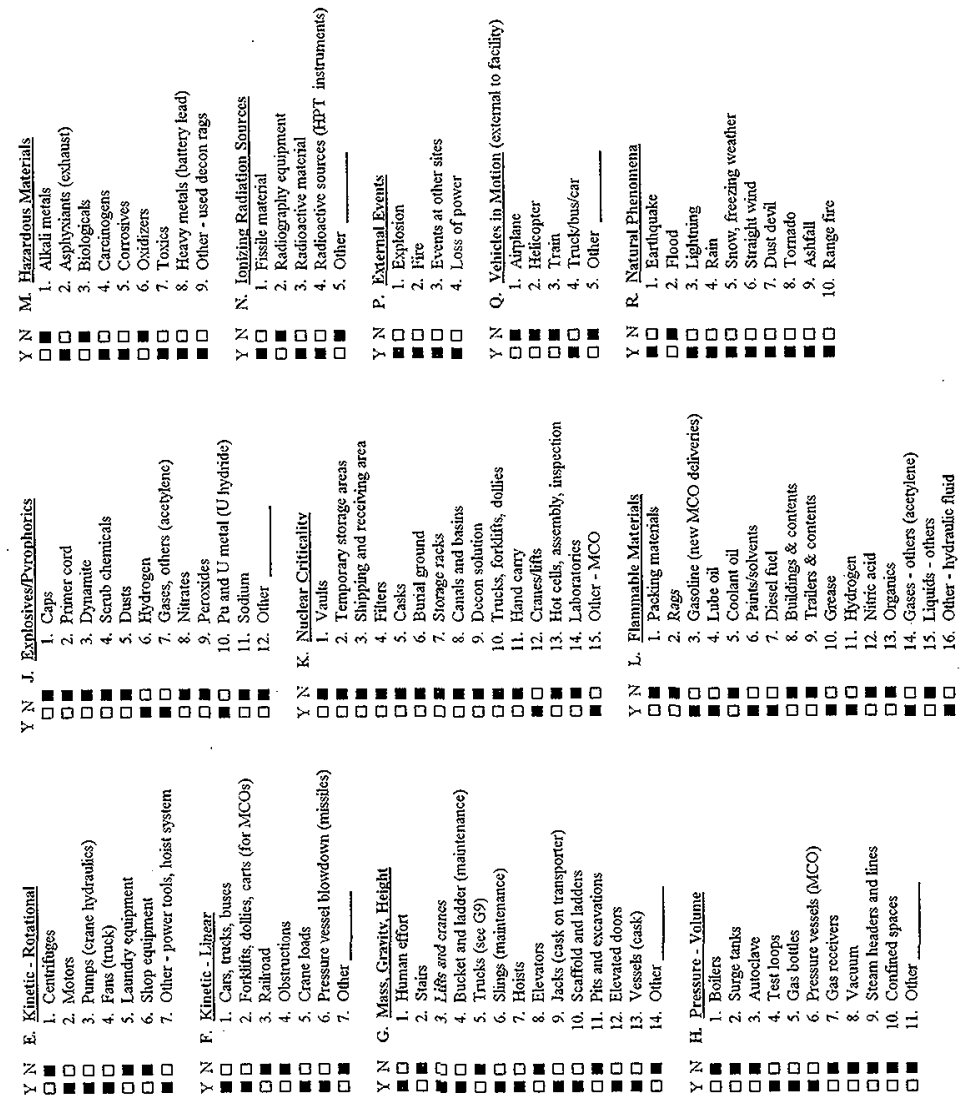

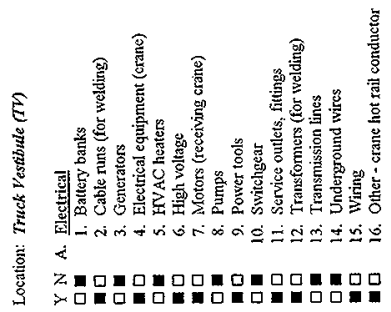

hie001rl wpd

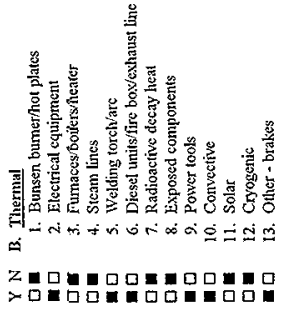

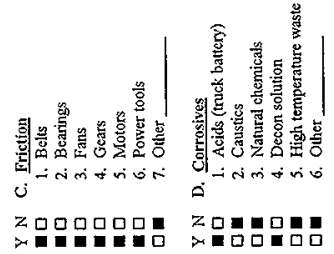

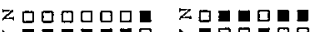

February 11, 1999 

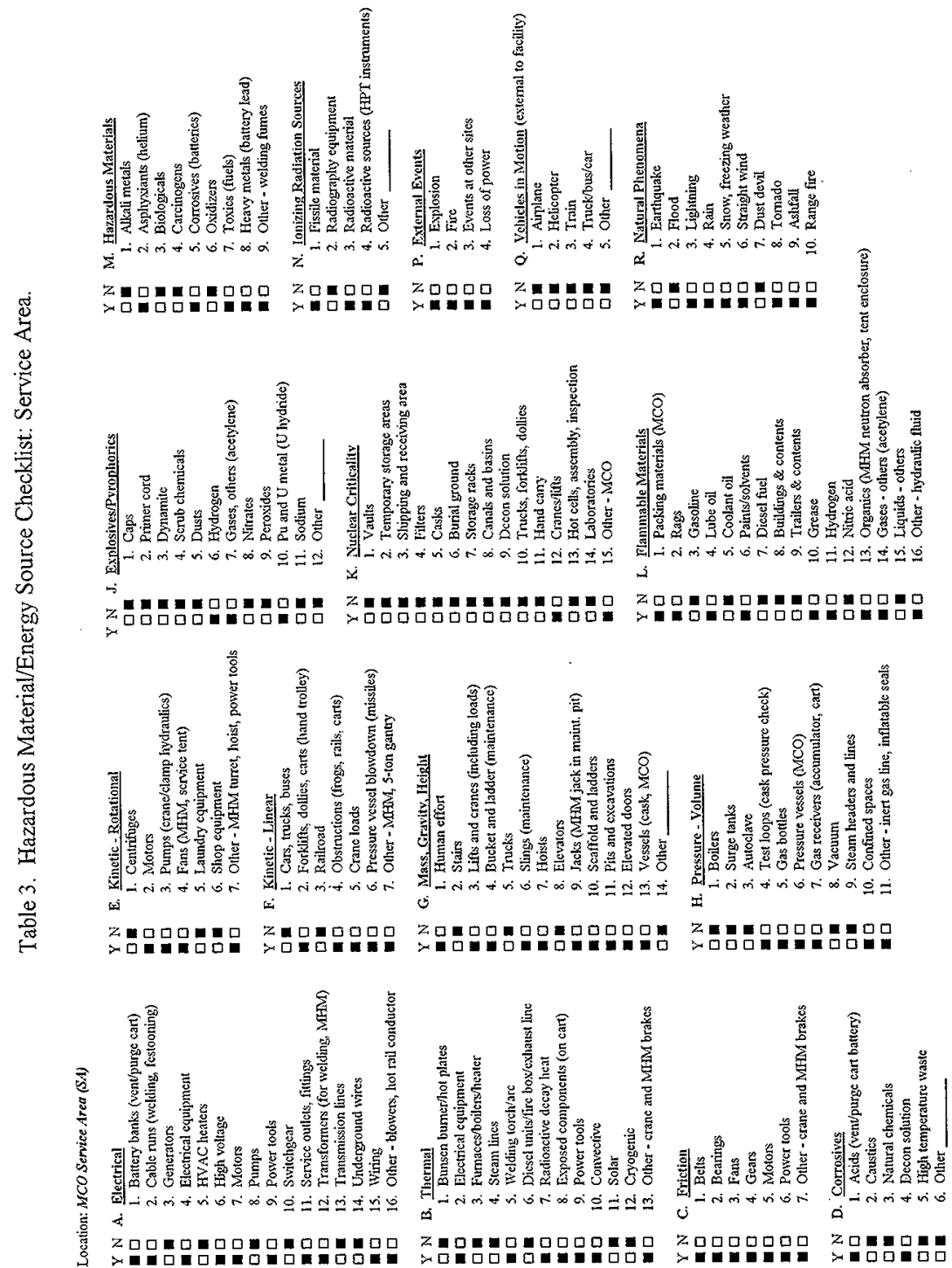


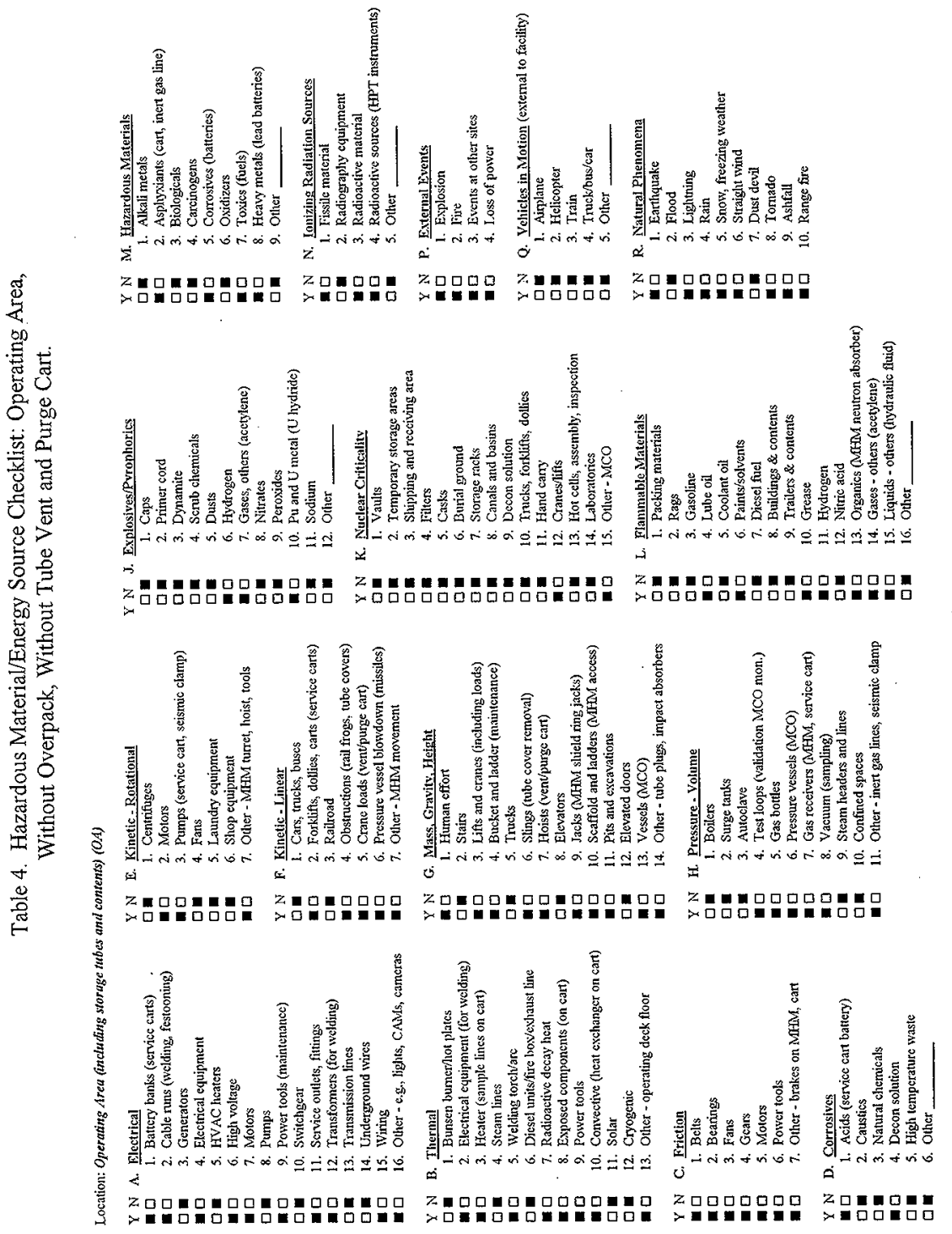




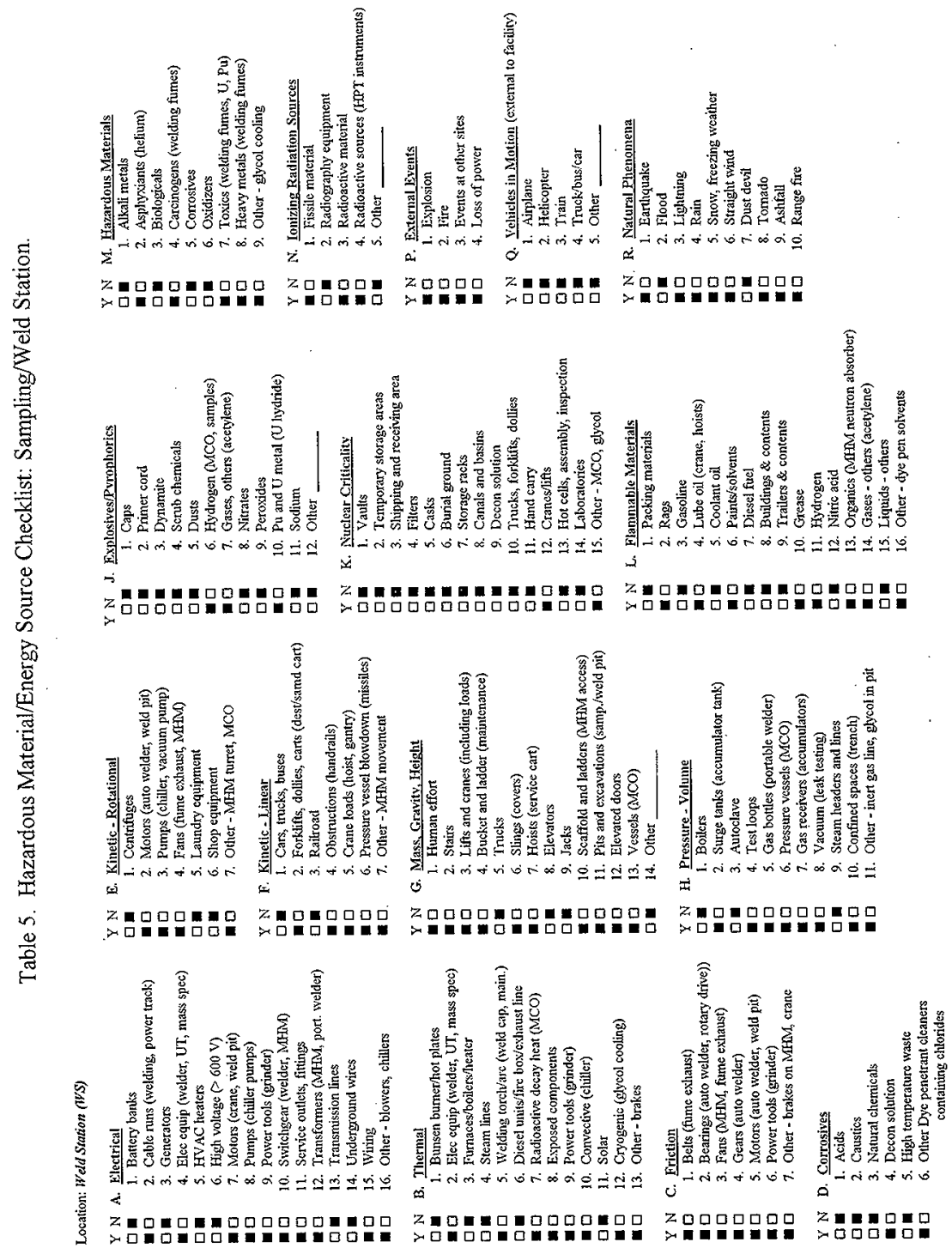



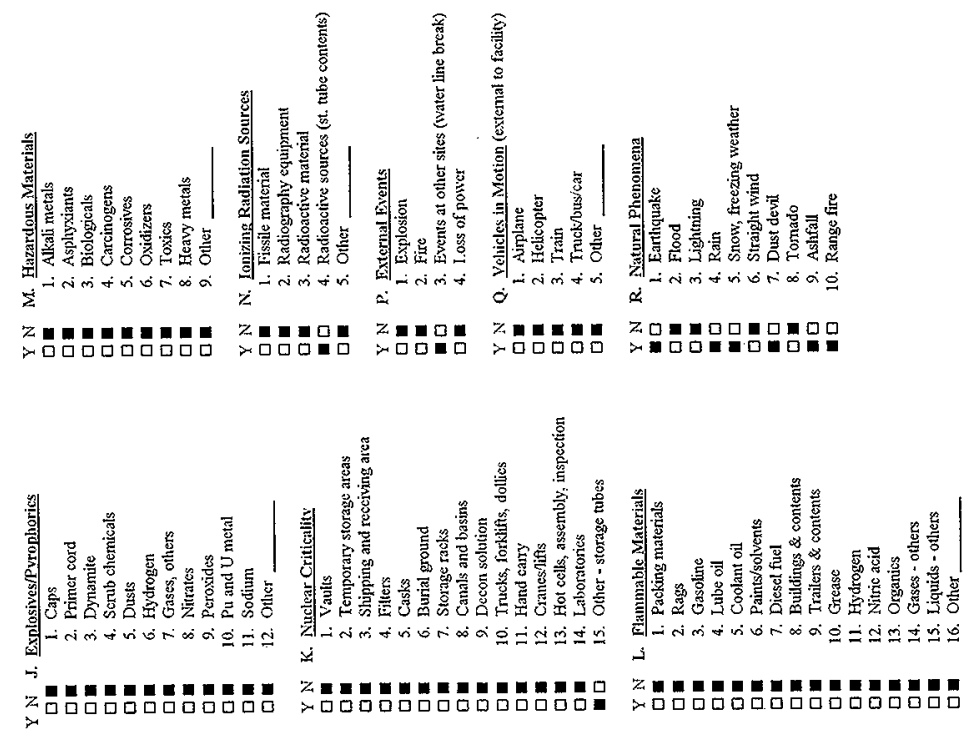

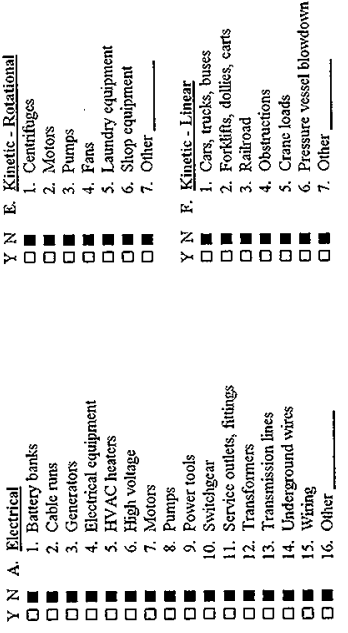

hie00lrl.wpd

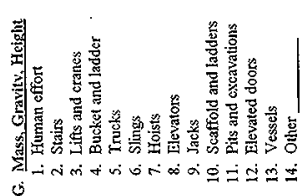
궁ํำ

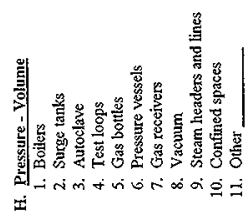

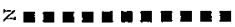
ㄱำํํำㅁำㅁำ

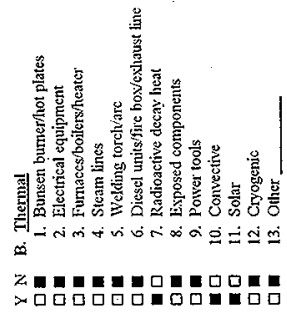



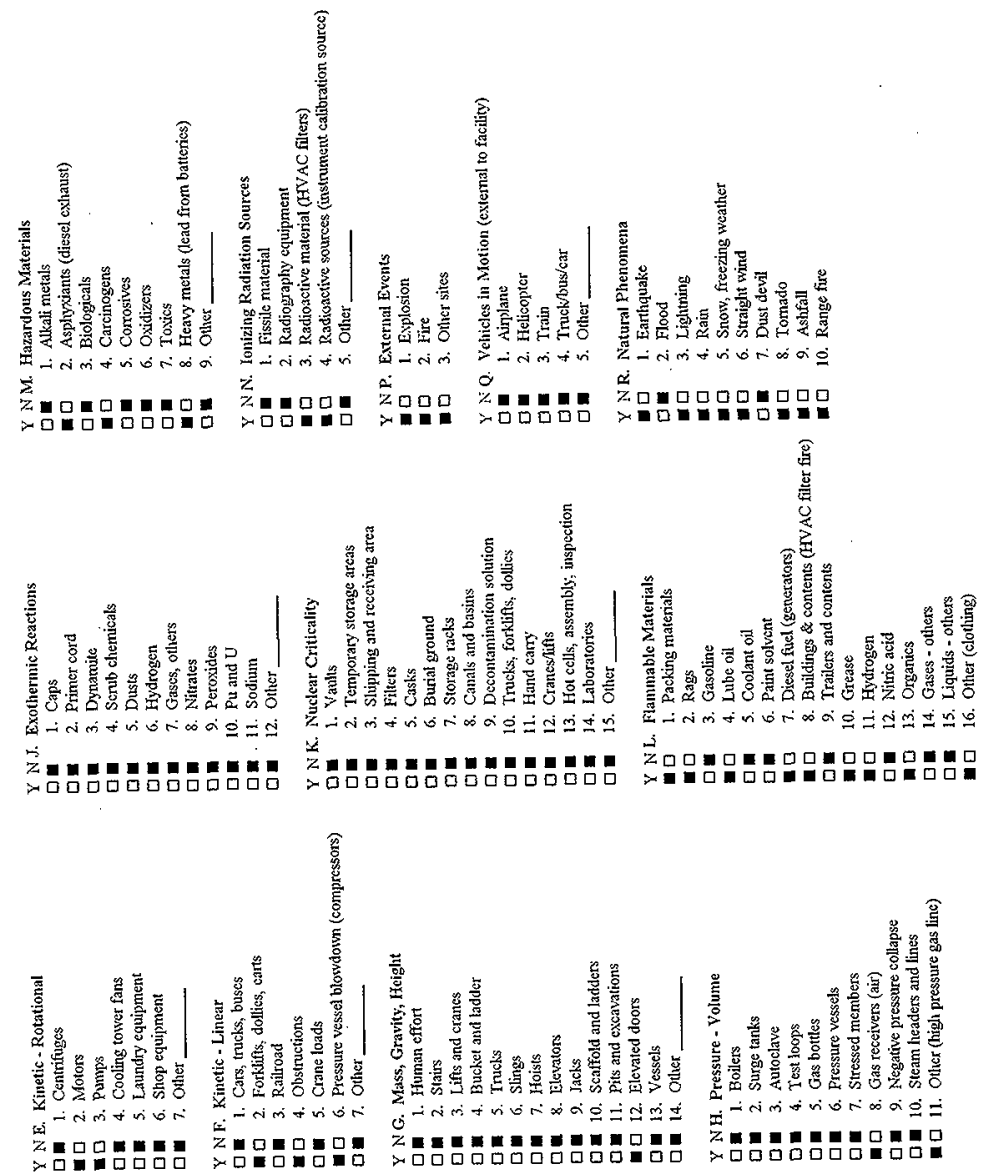

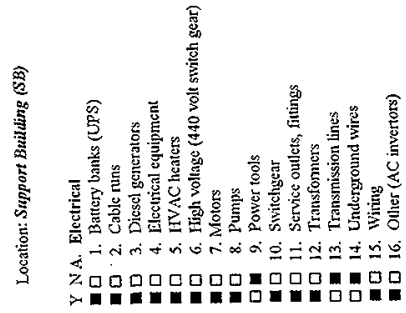

hie001rl.wpd

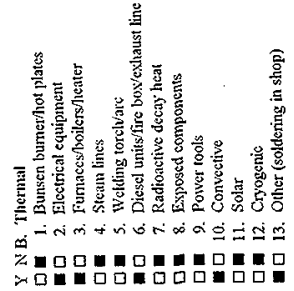

19

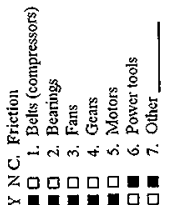

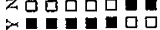

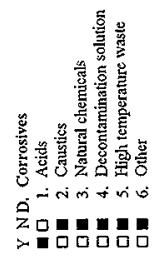

February 11, 1999 

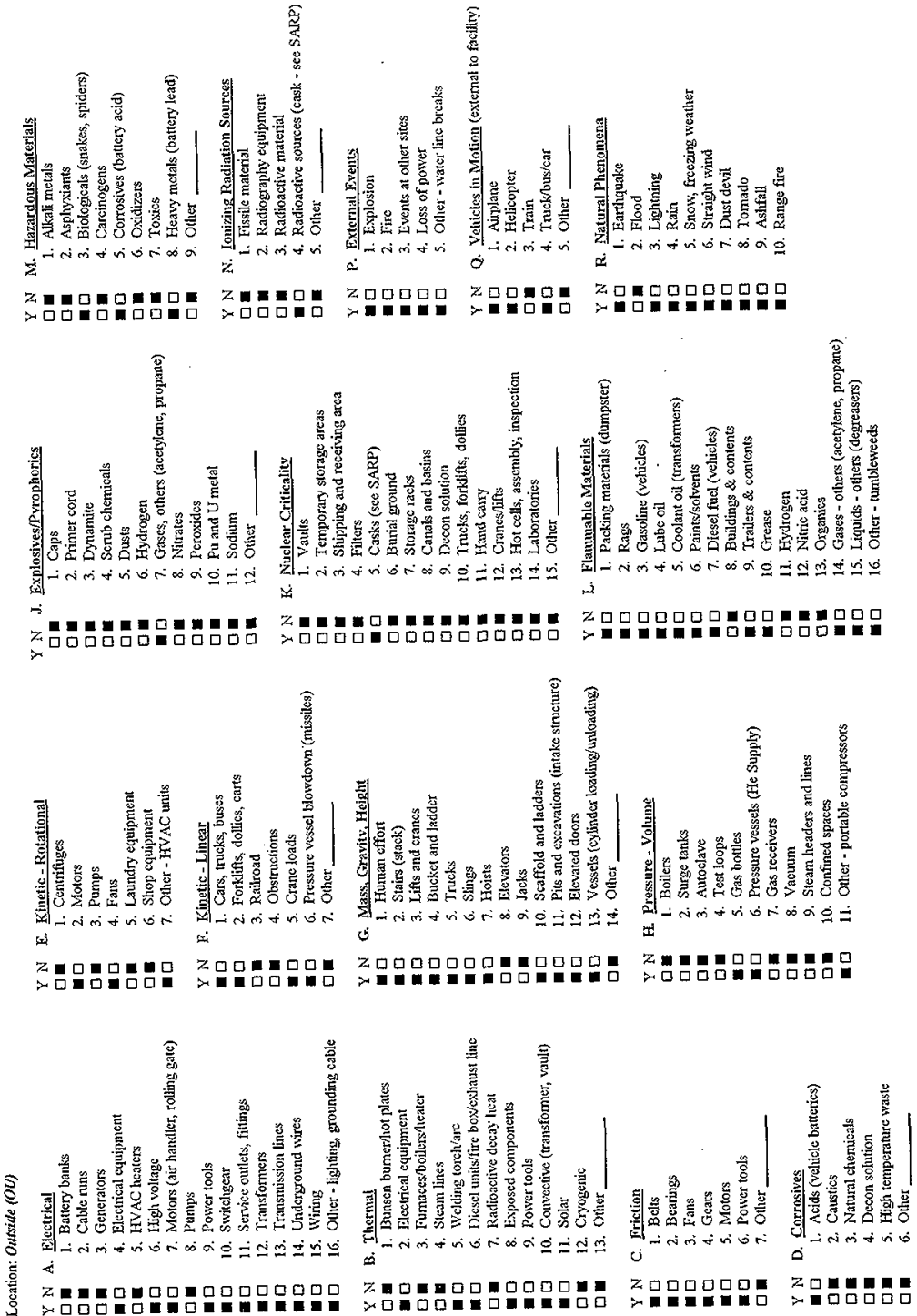
Table 9. Standard Industrial Hazards: Truck Vestibule. (2 sheets)

\begin{tabular}{|c|c|c|c|c|}
\hline \multirow{2}{*}{$\begin{array}{l}\text { Hazard category } \\
\text { Electrical }\end{array}$} & \multirow{2}{*}{$\begin{array}{l}\text { Hazard type } \\
\text { Crane cabling (for welding) }\end{array}$} & \multicolumn{3}{|c|}{ Checklist designator } \\
\hline & & TV & A & 2 \\
\hline & Electrical equipment (crane) & TV & A & 4 \\
\hline & High voltage & TV & A & 6 \\
\hline & Motors (receiving crane) & TV & A & 7 \\
\hline & Power tools & TV & A & 9 \\
\hline & Service outlets, fittings & TV & A & 11 \\
\hline & Transformers (for welding) & TV & A & 12 \\
\hline & Wiring & TV & A & 15 \\
\hline & Other - crane hot rail conductor & TV & A & 16 \\
\hline \multirow[t]{6}{*}{ Thermal } & Electrical equipment & TV & $\mathrm{B}$ & 2 \\
\hline & Welding, torch/arc (maintenance) & TV & B & 5 \\
\hline & Diesel units; exhaust lines on vehicles & TV & $\mathrm{B}$ & 6 \\
\hline & Power tools & TV & $\mathrm{B}$ & 9 \\
\hline & Convective heat from transportation cask & TV & B & 10 \\
\hline & Vehicle brakes & TV & $\mathrm{B}$ & 13 \\
\hline \multirow[t]{6}{*}{ Friction } & Belts & TV & $\mathrm{C}$ & 1 \\
\hline & Bearings & TV & $c$ & 2 \\
\hline & Fans & TV & $\mathrm{C}$ & 3 \\
\hline & Gears & TV & $\mathrm{C}$ & 4 \\
\hline & Motors & TV & $\mathrm{C}$ & 5 \\
\hline & Power tools & TV & $\mathrm{C}$ & 6 \\
\hline \multirow[t]{2}{*}{ Corrosives } & Vehicle battery acid & TV & $\mathrm{D}$ & I \\
\hline & Decontamination solution & TV & D & 4 \\
\hline \multirow[t]{4}{*}{ Kinetic - rotational } & Crane motor & TV & $E$ & 2 \\
\hline & Pumps (crane hydraulics) & TV & $\mathrm{E}$ & 3 \\
\hline & Fans (truck) & TV & $\mathrm{E}$ & 4 \\
\hline & Other - power tools, hoist system & $T V$ & $E$ & 7 \\
\hline \multirow[t]{6}{*}{ Mass, gravity, height } & Human effort (dropped items) & TV & $\mathrm{G}$ & I \\
\hline & Bucket and ladder (maintenance) & TV & G & 4 \\
\hline & Slings (maintenance) & TV & $\mathrm{G}$ & 6 \\
\hline & Hoists & TV & $\mathrm{G}$ & 7 \\
\hline & Scaffold and ladders & TV & G & 10 \\
\hline & Elevated doors & TV & $\mathrm{G}$ & 12 \\
\hline Pressure - volume & Gas bottles & TV & $\mathrm{H}$ & 5 \\
\hline
\end{tabular}


HNF-SD-SNF-HIE-001 REV 1

Table 9. Standard Industrial Hazards: Truck Vestibule. (2 sheets)

\begin{tabular}{|c|c|c|c|c|}
\hline Hazard category & Hazard type & \multicolumn{3}{|c|}{ Checklist designator } \\
\hline \multirow[t]{5}{*}{ Hazardous materials } & Asphyxiants (exhaust, helium line) & TV & M & 2 \\
\hline & Carcinogens & TV & $M$ & 4 \\
\hline & Corrosives (battery acid) & TV & M & 5 \\
\hline & Heavy metals (battery lead) & TV & M & 8 \\
\hline & Other - used decontamination solution & TV & $\mathrm{M}$ & 9 \\
\hline
\end{tabular}


Table 10. Standard Industrial Hazards: Service Area. (2 sheets)

\begin{tabular}{|c|c|c|c|c|}
\hline Hazard category & Hazard type & \multicolumn{3}{|c|}{ Checklist designator } \\
\hline \multirow[t]{10}{*}{ Electrical } & Battery banks (vent and purge cart) & SA & A & 1 \\
\hline & Cable runs (welding, festooning) & $\mathrm{SA}$ & A & 2 \\
\hline & Electrical equipment & $\mathrm{SA}$ & A & 4 \\
\hline & High voltage & SA & A & 6 \\
\hline & Motors & SA & $\mathrm{A}$ & 7 \\
\hline & Power tools & SA & A & 9 \\
\hline & Service outlets, fittings & $\mathrm{SA}$ & A & 11 \\
\hline & Transiormers (for welding) & $\mathrm{SA}$ & A & 12 \\
\hline & Wiring & SA & $\mathrm{A}$ & 15 \\
\hline & Other (blowers, hot rail conductor) & SA & A & 16 \\
\hline \multirow[t]{7}{*}{ Thermal } & Electrical equipment & $\overline{S A}$ & $\mathrm{~B}$ & 2 \\
\hline & Welding, torch/atc & SA & $B$ & 5 \\
\hline & Radioactive decay heat & SA & B & 7 \\
\hline & Exposed components (on cart) & $\mathrm{SA}$ & $\mathrm{B}$ & 8 \\
\hline & Power tools (no cutting tools) & SA & $\mathrm{B}$ & 9 \\
\hline & Convective & SA & $\mathrm{B}$ & 10 \\
\hline & Other (crane and MHM brakes) & SA & $\mathrm{B}$ & 13 \\
\hline \multirow[t]{7}{*}{ Friction } & Beits & $\mathrm{SA}$ & $\mathrm{C}$ & 1 \\
\hline & Bearings & SA & $\mathrm{C}$ & 2 \\
\hline & Fans & SA & $\mathrm{C}$ & 3 \\
\hline & Gears & $\mathrm{SA}$ & $\mathrm{C}$ & 4 \\
\hline & Motors & SA & $\mathrm{C}$ & 5 \\
\hline & Power tools & $\mathrm{SA}$ & $\mathrm{C}$ & 6 \\
\hline & Other (crane and MHM brakes) & $\mathrm{SA}$ & $\mathrm{C}$ & 7 \\
\hline \multirow[t]{2}{*}{ Corrosives } & Acids (vent and purge cart battery) & $\mathrm{SA}$ & $\mathrm{D}$ & 1 \\
\hline & Decontamination solution & SA & $D$ & 4 \\
\hline \multirow[t]{4}{*}{ Kinetic - rotational } & Motors & SA & $\mathrm{E}$ & 2 \\
\hline & Pumps (crane and clamp hydraulics) & $\mathrm{SA}$ & $\mathrm{E}$ & 3 \\
\hline & Fans (MHM, service tent) & $\mathrm{SA}$ & $\mathrm{E}$ & 4 \\
\hline & Other -5 -ton hoist & SA & $\mathrm{E}$ & 7 \\
\hline \multirow[t]{2}{*}{ Kinetic - linear } & Forklifts, dollies, carts (hand trolley) & SA & $\mathrm{F}$ & 2 \\
\hline & Obstructions (frogs) & SA & $\mathrm{F}$ & 4 \\
\hline
\end{tabular}


Table 10. Standard Industrial Hazards: Service Area. (2 sheets)

\begin{tabular}{|c|c|c|c|c|}
\hline Hazard category & Hazard type & \multicolumn{3}{|c|}{ Checklist designator } \\
\hline \multirow[t]{7}{*}{ Mass, gravity, height } & Human effort & SA & $\mathrm{G}$ & 1 \\
\hline & Bucket and ladder (maintenamce) & $\mathrm{SA}$ & $\mathrm{G}$ & 4 \\
\hline & Slings (maintenance) & SA & G & 6 \\
\hline & Hoists & $\mathrm{SA}$ & $\mathrm{G}$ & 7 \\
\hline & Jacks (MH-M jack in maintenance pit) & $\mathrm{SA}$ & $\mathrm{G}$ & 9 \\
\hline & Scaffolds and ladders & SA & $\mathrm{G}$ & 10 \\
\hline & Elevated doors & $\mathrm{SA}$ & $\mathrm{G}$ & 12 \\
\hline \multirow[t]{4}{*}{ Pressure - volume } & Gas bottles & SA & $\mathrm{H}$ & 5 \\
\hline & Gas receivers (accumulator, cart) & $S A$ & $\mathrm{H}$ & 7 \\
\hline & Confined spaces & $\mathrm{SA}$ & $\mathrm{H}$ & 10 \\
\hline & Other - pressurized inert gas line & SA & $\mathrm{H}$ & 11 \\
\hline \multirow[t]{2}{*}{ Hazardous materials } & Corrosives (batteries) & $\mathrm{SA}$ & $\mathrm{M}$ & 5 \\
\hline & Heavy metals (battery lead) & $\mathrm{SA}$ & $\mathrm{M}$ & 8 \\
\hline $\begin{array}{l}\text { Ionizing radiation } \\
\text { Sources }\end{array}$ & Radioactive sources (hand-held equipment) & SA & $\mathrm{N}$ & 4 \\
\hline
\end{tabular}

MHM = multi-canister overpack handling machine. 
Table 11. Standard Industrial Hazards: Operating Area. (2 sheets)

\begin{tabular}{|c|c|c|c|c|}
\hline \multirow{2}{*}{$\frac{\text { Hazard category }}{\text { Electrical }}$} & \multirow{2}{*}{$\begin{array}{l}\text { Hazard type } \\
\text { Battery banks (service carts) }\end{array}$} & \multicolumn{3}{|c|}{ Checklist designator } \\
\hline & & $\mathrm{OA}$ & A & 1 \\
\hline & Cable runs (welding, lestooning) & OA & A & 2 \\
\hline & High voltage & $\mathrm{OA}$ & A & 6 \\
\hline & Motors & OA & A & 7 \\
\hline & Power tools (maintenance) & OA & A & 9 \\
\hline & Service outlets, fittings & $\mathrm{OA}$ & A & 11 \\
\hline & Transformers (welding) & $\mathrm{OA}$ & A & 12 \\
\hline & Wiring & $\mathrm{OA}$ & A & 15 \\
\hline & Other - lighting, CAMs, cameras & OA & A & 16 \\
\hline \multirow[t]{8}{*}{ Thermal } & Electrical equipment (for welding) & OA & $\mathrm{B}$ & 2 \\
\hline & Heater (sample lines on cart) & OA & $\mathrm{B}$ & 3 \\
\hline & Welding, torclvarc & $\mathrm{OA}$ & $\mathrm{B}$ & 5 \\
\hline & Radioactive decay heat & OA & $\mathrm{B}$ & 7 \\
\hline & Exposed components (on cart) & $\mathrm{OA}$ & $B$ & 8 \\
\hline & Power tools & $\mathrm{OA}$ & $\mathrm{B}$ & 9 \\
\hline & Convective (heat exchanger on cart) & $\mathrm{OA}$ & $\mathrm{B}$ & 10 \\
\hline & Other - operating deck floor & $\mathrm{OA}$ & $\mathrm{B}$ & 13 \\
\hline \multirow[t]{7}{*}{ Friction } & Belts & $\mathrm{OA}$ & $\mathrm{C}$ & 1 \\
\hline & Bearings & $\mathrm{OA}$ & $\mathrm{C}$ & 2 \\
\hline & Fans & $\mathrm{OA}$ & $\mathrm{C}$ & 3 \\
\hline & Gears & $\mathrm{OA}$ & $\mathrm{C}$ & 4 \\
\hline & Motors & $\mathrm{OA}$ & $\mathrm{C}$ & 5 \\
\hline & Power tools & $\mathrm{OA}$ & $\mathrm{C}$ & 6 \\
\hline & Other (brakes on the MHM and tube cart) & $\mathrm{OA}$ & C & 7 \\
\hline Corrosives & Decontamination solution & $\mathrm{OA}$ & $\mathrm{D}$ & 4 \\
\hline \multirow[t]{3}{*}{ Kinetic-rotational } & Motors & $\mathrm{OA}$ & $\mathrm{E}$ & 2 \\
\hline & Pumps (service cart, seismic clamp) & $\mathrm{OA}$ & $\mathrm{E}$ & 3 \\
\hline & Other - MHM Moist & OA & $\mathrm{E}$ & 7 \\
\hline \multirow[t]{3}{*}{ Kinetic - linear } & Forklifts, dollies, carts (service carts) & OA. & $\mathrm{F}$ & 2 \\
\hline & Crane loads (service carts) & $\mathrm{OA}$ & $\mathrm{F}$ & 5 \\
\hline & $\begin{array}{l}\text { Pressure vessel blowdow'n (maintenance, gas bottles } \\
\text { as missiles) }\end{array}$ & OA. & $\mathrm{F}$ & 6 \\
\hline
\end{tabular}


HNF-SD-SNF-HIE-001 REV 1

Table 11. Standard Industrial Hazards: Operating Area. (2 sheets)

\begin{tabular}{|l|l|lll|}
\hline \multicolumn{1}{|c|}{ Hazard category } & \multicolumn{1}{|c|}{ Hazard type } & \multicolumn{3}{c|}{ Checklist designator } \\
\hline \multirow{5}{*}{ Mass, gravity, height } & Human effort & OA & G & 1 \\
\cline { 2 - 5 } & Bucket and ladder (maintenance) & OA & G & 4 \\
\cline { 2 - 5 } & Slings (to remove tube plug cover) & OA & G & 6 \\
\cline { 2 - 5 } & Hoists (vent and purge cart) & OA & G & 7 \\
\cline { 2 - 5 } & Jacks (MHM shield ring jacks) & OA & G & 9 \\
\cline { 2 - 5 } & Scaffold and ladders (MHM access) & OA & G & 10 \\
\cline { 2 - 5 } & Pits and excavations & OA & G & 11 \\
\hline \multirow{5}{*}{ Pressure - volume } & Gas bottles & OA & H & 5 \\
\cline { 2 - 5 } & Gas receivers (MHM, service cart) & OA & H & 7 \\
\cline { 2 - 5 } & Other - inert gas lines, seismic clamp & OA & H & 11 \\
\hline Hazardous materials & Corrosives (batteries) & OA & M & 5 \\
\cline { 2 - 5 } & Heavy metals (lead batteries) & OA & M & 8 \\
\hline Ionizing radiation sources & Radioactive sources (hand-held equipment) & N & 4 \\
\hline
\end{tabular}

$\mathrm{CAM}=$ continuous air monitor.

$\mathrm{MHM}=$ multi-canister overpack handling machine. 
Table 12. Standard Industrial Hazards: Sampling/Weld Station. (2 sheets)

\begin{tabular}{|c|c|c|c|c|}
\hline Hazard category & Hazard type & \multicolumn{3}{|c|}{ Checklist designator } \\
\hline \multirow[t]{10}{*}{ Electrical } & Cable runs (welding, power track) & WS & A & 2 \\
\hline & $\begin{array}{l}\text { Electrical equipment (welder, UT, mass } \\
\text { spectrometer) }\end{array}$ & WS & A & 4 \\
\hline & Motors (crane, weld pit) & WS & A & 7 \\
\hline & Pumps (chiller pumps) & WS & A & 8 \\
\hline & Power tools (grinder) & WS & A & 9 \\
\hline & Switchgear (welder, MHM) & WS & A & 10 \\
\hline & Service outlets, fittings & WS & A & 11 \\
\hline & Transformers (MHM, portable welder) & WS & A & 12 \\
\hline & Wiring & WS & A & 15 \\
\hline & Other - blowers, chillers & WS & A & 16 \\
\hline \multirow[t]{7}{*}{ Thermal } & $\begin{array}{l}\text { Electrical equipment (welder, UT, mass } \\
\text { spectrometer) }\end{array}$ & WS & $\mathrm{B}$ & 2 \\
\hline & Welding, torcl/arc (weld cap, maintenance) & WS & B & 5 \\
\hline & $\begin{array}{l}\text { Exposed components (shielding, exposed top of } \\
\text { MCO) }\end{array}$ & WS & B & 8 \\
\hline & Power tools (grinder) & WS & B & 9 \\
\hline & Convective (chiller) & WS & B & 10 \\
\hline & Cryogenic (glycol cooling) & WS & B & 12 \\
\hline & Other - brakes (MHM, gantry, hoists) & WS & $\mathrm{B}$ & 13 \\
\hline \multirow[t]{7}{*}{ Friction } & Belts (fume exhaust) & WS & $\mathrm{C}$ & 1 \\
\hline & Bearings (auto welder, kotary drive) & WS & $\mathrm{C}$ & 2 \\
\hline & Fans (MHM, fume exhaust) & WS & $\mathrm{C}$ & 3 \\
\hline & Gears (auto welder) & WS & $\mathrm{C}$ & 4 \\
\hline & Motors (auto welder, weld pit) & WS & $\mathrm{C}$ & 5 \\
\hline & Power tools (grinder) & WS & $\mathrm{C}$ & 6 \\
\hline & Other - brakes on the MHM and crane & WS & $\mathrm{C}$ & 7 \\
\hline \multirow[t]{3}{*}{ Kinetic - rotational } & Motors (auto welder, weld pit) & WS & $\mathrm{E}$ & 2 \\
\hline & Pumps (chiller, vacuum pump) & WS & $\mathrm{E}$ & 3 \\
\hline & Fans (fume exhaust, MIIM) & WS & $E$ & 4 \\
\hline Kinetic - linear & Obstructions (handrails) & WS & $\mathrm{F}$ & 4 \\
\hline
\end{tabular}


Table 12. Standard Industrial Hazards: Sampling/Weld Station. (2 sheets)

\begin{tabular}{|l|l|lll|}
\hline \multicolumn{1}{|c|}{ Hazard category } & \multicolumn{1}{|c|}{ Hazard type } & \multicolumn{3}{c|}{ Checklist designator } \\
\hline \multirow{4}{*}{ Mass, gravity, height } & Stairs & WS & G & 2 \\
\cline { 2 - 4 } & Scaffold and ladders (MHM access) & WS & G & 10 \\
\cline { 2 - 4 } & Pits and excavations (sample/weld pit) & WS & G & 11 \\
\cline { 2 - 4 } & Elevated doors & WS & G & 12 \\
\hline Pressure - volume & Gas bottles & WS & H & 5 \\
\cline { 2 - 5 } & Confined spaces (trench) & WS & H & 10 \\
\cline { 2 - 5 } & Other - inert gas lines, glycol in pit & WS & H & 11 \\
\hline \multirow{3}{*}{ Hazardous materials } & Carcinogens (welding fumes) & WS & M & 4 \\
\cline { 2 - 5 } & Heavy metals (welding fumes) & WS & N \\
\hline Ionizing radiation sources & Radioactive sources (hand-held equipment) & & \\
\hline
\end{tabular}

$\mathrm{MCO}=$ multi-canister overpack.

$\mathrm{MHM}=$ multi-canister overpack handling machine.

UT $=$ ultrasonic test. 
Table 13. Standard Industrial Hazards: Support Building. (2 sheets)

\begin{tabular}{|c|c|c|c|c|}
\hline \multirow{2}{*}{$\frac{\text { Hazard category }}{\text { Electrical }}$} & \multirow{2}{*}{$\frac{\text { Hazard type }}{\text { Battery banks (uninterruptible power supply) }}$} & \multicolumn{3}{|c|}{ Checklist designator } \\
\hline & & $\mathrm{SB}$ & A & 1 \\
\hline \multirow{12}{*}{$\cdot$} & Cable runs & SB & A & 2 \\
\hline & Electrical equipment & SB & A & 4 \\
\hline & HVAC heaters & $\mathrm{SB}$ & A & 5 \\
\hline & High voltage $(>600 \mathrm{~V})$ & $\mathrm{SB}$ & A & 6 \\
\hline & Motors & $\mathrm{SB}$ & A & 7 \\
\hline & Pumps & $\mathrm{SB}$ & A & 8 \\
\hline & Power tools & $\mathrm{SA}$ & A & 9 \\
\hline & Switchgear $(440 \mathrm{~V})$ & $\mathrm{SB}$ & $\mathrm{A}$ & 10 \\
\hline & Service outlets, fittings & $\mathrm{SB}$ & A & 11 \\
\hline & Transformers & SB & A & 12 \\
\hline & Wiring & SB & A & 15 \\
\hline & Other ( $\mathrm{AC}$ invertors) & SB & A & 16 \\
\hline \multirow[t]{5}{*}{ Thermal } & Electrical equipment & $\mathrm{SB}$ & $B$ & 2 \\
\hline & Furnaces, boilers, heater & SB & B & 3 \\
\hline & Welding, torch/arc (maintenance) & SB & $\mathrm{B}$ & 5 \\
\hline & Power tools & SB & $\mathrm{B}$ & 9 \\
\hline & Other (soldering in shop) & $\mathrm{SB}$ & $\mathrm{B}$ & 13 \\
\hline \multirow[t]{6}{*}{ Friction } & Belts (exhaust fans) & SB & $\mathrm{C}$ & 1 \\
\hline & Bearings & $\mathrm{SB}$ & $\mathrm{C}$ & 2 \\
\hline & Fans & SB & C & 3 \\
\hline & Gears & SB & $\mathrm{C}$ & 4 \\
\hline & Motors & $\mathrm{SB}$ & C & 5 \\
\hline & Power tools & $\mathrm{SB}$ & $\mathrm{C}$ & 6 \\
\hline \multirow[t]{2}{*}{ Corrosives } & Acids (battery banks) & $\mathrm{SB}$ & $\mathrm{D}$ & 1 \\
\hline & Decontamination solution & $S B$ & D & 4 \\
\hline \multirow[t]{4}{*}{ Kinetic - rotational } & Motors & $\mathrm{SB}$ & $\mathrm{E}$ & 2 \\
\hline & Pumps & $\mathrm{SB}$ & $\mathrm{E}$ & 3 \\
\hline & Fans (HVAC fans) & $\mathrm{SB}$ & $\mathrm{E}$ & 4 \\
\hline & Other - compressors, power tools & $\mathrm{SB}$ & $\mathrm{E}$ & 7 \\
\hline
\end{tabular}


-HNF-SD-SNF-HIE-001 REV 1

Table 13. Standard Industrial Hazards: Support Building. (2 sheets)

\begin{tabular}{|c|c|c|c|c|}
\hline \multirow{2}{*}{$\begin{array}{l}\text { Hazard category } \\
\text { Kinetic - linear }\end{array}$} & \multirow[t]{2}{*}{ Hazard type } & \multicolumn{3}{|c|}{ Checklist designator } \\
\hline & & TV & $\mathrm{F}$ & 1 \\
\hline & Crane loads & TV & $F$ & 5 \\
\hline & Cars, trucks, buses & SB & F & 1 \\
\hline & Forklifts, dollies, cauts & SB & $\mathrm{F}$ & 2 \\
\hline & Obstructions & SB & F & 4 \\
\hline & Crane loads & $\mathrm{SB}$ & $\mathrm{F}$ & 5 \\
\hline & Pressure vessel blowdown (missiles) & SB & $\mathrm{F}$ & 6 \\
\hline \multirow[t]{6}{*}{ Mass, gravity, height } & Human effort & SB & $G$ & 1 \\
\hline & Slings & $\mathrm{SB}$ & $\mathrm{G}$ & 6 \\
\hline & Hoists & SB & $\mathrm{G}$ & 7 \\
\hline & Scaffold and ladders & SB & $\mathrm{G}$ & 10 \\
\hline & Pits and excavations (sumps) & SB & $\mathrm{G}$ & 11 \\
\hline & Elevated doors & $\mathrm{SB}$ & $\mathrm{G}$ & 12 \\
\hline \multirow[t]{5}{*}{ Pressure - volume } & Gas bottles & SB & $\mathrm{H}$ & 5 \\
\hline & Pressure vessels (air receiver, $125 \mathrm{lb} / \mathrm{in}^{2}$ gauge) & SB & $\mathrm{H}$ & 6 \\
\hline & Gas receivers (air) & SB & $\mathrm{H}$ & 7 \\
\hline & Vacuum (small pumps) & SB & $\mathrm{H}$ & 8 \\
\hline & Other (high pressure inert gas line) & SB & $\mathrm{H}$ & 11 \\
\hline \multirow[t]{5}{*}{ Hazardous materials } & Asphyxiants (vehicle exhaust) & SB & M & 2 \\
\hline & Carcinogens (fumes) & SB & $\mathrm{M}$ & 4 \\
\hline & Corrosives (decontamination solution, batteries) & SB & M & 5 \\
\hline & Heavy metals (lead from batteries) & SB & M & 8 \\
\hline & Other - cleaning supplies & $\mathrm{SB}$ & M & 9 \\
\hline \multirow[t]{2}{*}{ Ionizing radiation sources } & Radioactive material (decontamination rags, filters) & $\mathrm{SB}$ & $N$ & 3 \\
\hline & Radioactive sources (instrument calibration sources) & SB & $N$ & 4 \\
\hline
\end{tabular}

$\mathrm{HVAC}=$ heating, ventilation, and air conditioning. 
HNF-SD-SNF-HIE-001 REV 1

Table 14. Standard Industrial Hazards: Outside. (2 sheets)

\begin{tabular}{|c|c|c|c|c|}
\hline \multirow{2}{*}{$\frac{\text { Hazard category }}{\text { Electrical }}$} & \multirow{2}{*}{$\begin{array}{l}\text { Hazard type } \\
\text { Electrical equipment }\end{array}$} & \multicolumn{3}{|c|}{ Checklist designator } \\
\hline & & OU & A & 4 \\
\hline & High voltage & OU & A & 6 \\
\hline & Motors (air handlers, rolling gate) & OU & A & 7 \\
\hline & Power tools & OU & A & 9 \\
\hline & Switchgear & OU & A & 10 \\
\hline & Service outlets, fittings & OU & A & 11 \\
\hline & Transformers & OU & A & 12 \\
\hline & Transmission lines & OU & A & 13 \\
\hline & Underground wires & OU & A & 14 \\
\hline & Wiring & OU & $\mathrm{A}$ & 15 \\
\hline & Other - lighting, grounding cable & OU & A & 16 \\
\hline \multirow[t]{5}{*}{ Thermal } & Electrical equipment & OU & $\mathrm{B}$ & 2 \\
\hline & Diesel units, fire box, exhaust line & OU & $\mathrm{B}$ & 6 \\
\hline & Exposed components & OU & B & 8 \\
\hline & Convective & OU & $\mathrm{B}$ & 10 \\
\hline & Solar & $\mathrm{OU}$ & $\mathrm{B}$ & 11 \\
\hline \multirow[t]{6}{*}{ Friction } & Belts & OU & $\mathrm{C}$ & 1 \\
\hline & Bearings & OU & $\mathrm{C}$ & 2 \\
\hline & Fans & OU & $\mathrm{C}$ & 3 \\
\hline & Gears & OU. & C & 4 \\
\hline & Motors & OU & $\mathrm{C}$ & 5 \\
\hline & Power tools & OU & C & 6 \\
\hline Corrosives & Acids (vehicle batteries) & OU & $\mathrm{D}$ & 1 \\
\hline \multirow[t]{3}{*}{ Kinetic - rotational } & Motors & OU & $\mathrm{E}$ & 2 \\
\hline & Fans & OU & E & 4 \\
\hline & Other (heating, ventilation, and air conditioning units) & OU & $\mathrm{E}$ & 7 \\
\hline
\end{tabular}


HNF-SD-SNF-HIE-001 REV 1

Table 14. Standard Industrial Hazards: Outside. (2 sheets)

\begin{tabular}{|c|c|c|c|c|}
\hline \multirow{2}{*}{$\begin{array}{r}\text { Hazard category } \\
\text { Mass, gravity, height }\end{array}$} & \multirow[t]{2}{*}{ Hazard type } & \multicolumn{3}{|c|}{ Checklist designator } \\
\hline & & OU & G & 1 \\
\hline & Stairs (stack) & OU & G & 2 \\
\hline & Lifts and cranes & OU & G & 3 \\
\hline & Bucket and ladder & OU & $\mathrm{G}$ & 4 \\
\hline & Trucks & ou & $\mathrm{G}$ & 5 \\
\hline & Slings & OU & $\mathrm{G}$ & 6 \\
\hline & Hoists & $\mathrm{OU}$ & $\mathrm{G}$ & 7 \\
\hline & Scaffold and ladders & OU & $\mathrm{G}$ & 10 \\
\hline & Pits and excavations (air intake structures) & ov & $\mathrm{G}$ & 11 \\
\hline & Elevated doors & $\mathrm{OU}$ & $\mathrm{G}$ & 12 \\
\hline . & Vessels (cylinder loading and unloading) & OU & $\mathrm{G}$ & 13 \\
\hline \multirow[t]{3}{*}{ Pressure - volume } & Gas bottles & OU & $\mathrm{H}$ & 5 \\
\hline & Pressure vessels (helium supply) & OU & $\mathrm{H}$ & 6 \\
\hline & Other - portable compressors & OU & $\mathrm{H}$ & 11 \\
\hline $\begin{array}{l}\text { Explosives/ } \\
\text { pyrophorics }\end{array}$ & Gases, others (acetylene, propanc) & OU & j & 7 \\
\hline \multirow[t]{9}{*}{ Flammable materials } & Packing materials (dumpster) & OU & $L$ & 1 \\
\hline & Gasoline & OU & $\mathrm{L}$ & 3 \\
\hline & Lube oil & $\mathrm{OU}$ & L & 4 \\
\hline & Coolant oil (transformers) & OU & $\mathrm{L}$ & 5 \\
\hline & Paints and solvents & OU & L & 6 \\
\hline & Diesel fuel (vehicles) & OU & $\mathrm{L}$ & 7 \\
\hline & Grease & OU & L & 10 \\
\hline & Gases, others (acetylene, propane) & $\mathrm{OU}$ & $\mathrm{L}$ & 14 \\
\hline & Liquids, others (degreasers) & OU & L & 15 \\
\hline \multirow[t]{3}{*}{ Hazardous materials } & Biologicals (snakes, spiders) & OU & M & 3 \\
\hline & Corrosives (battery acid) & OU & M & 5 \\
\hline & Heavy metals (battery lead) & OU & M & 8 \\
\hline
\end{tabular}


HNF-SD-SNF-HIE-001 REV I

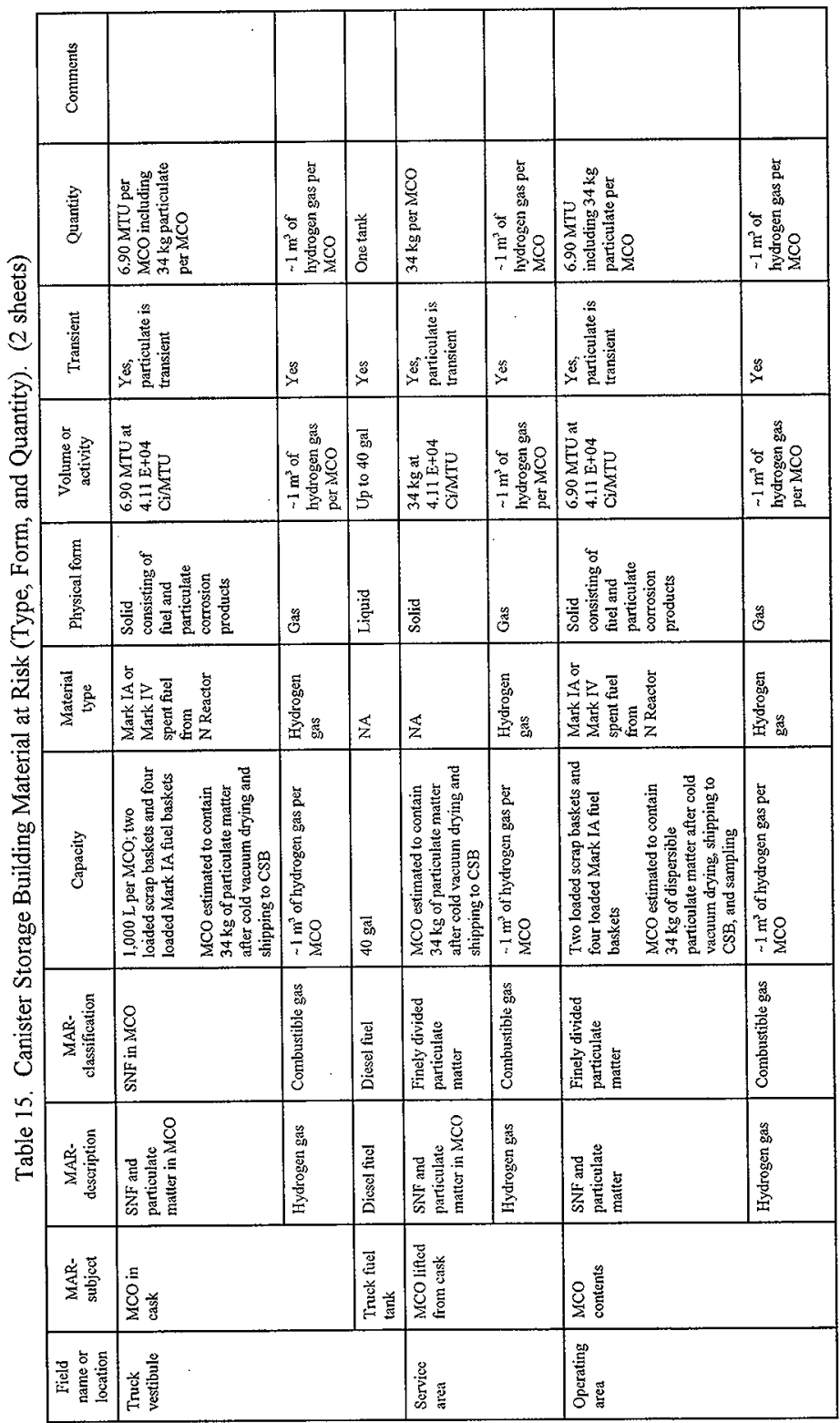


HNF-SD-SNF-HIE-001 REV 1

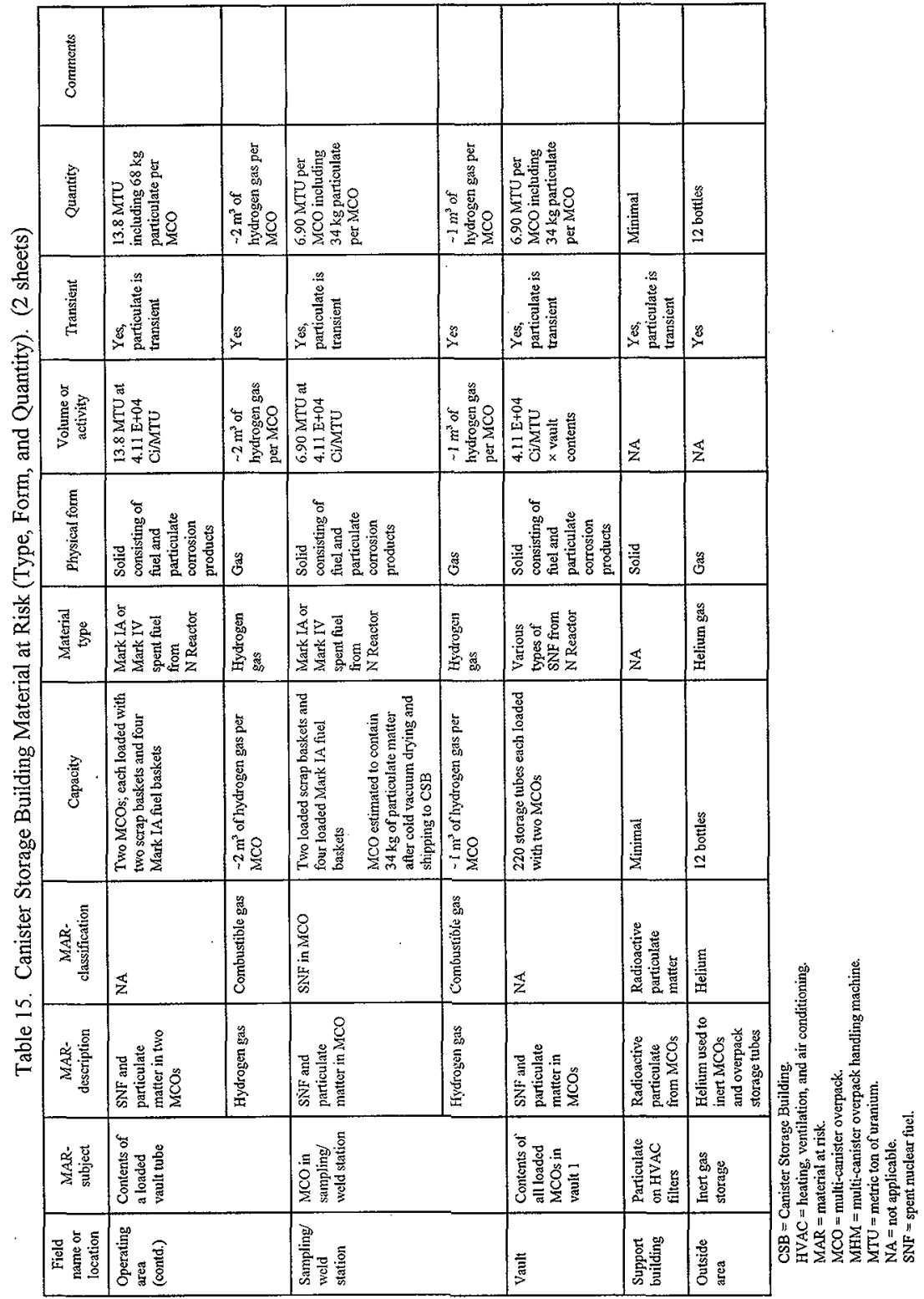

hie001rl.wpd 
HNF-SD-SNF-FIE-001 REV 1

\begin{tabular}{|c|c|}
\hline 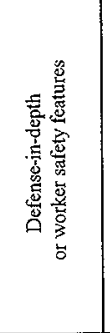 & 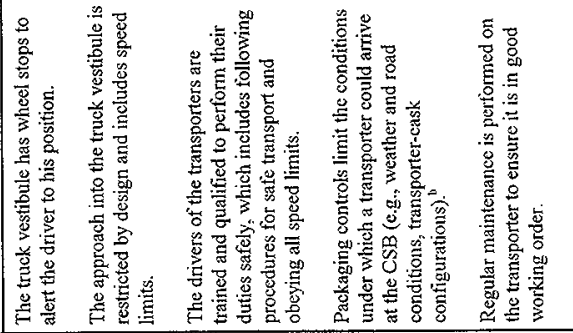 \\
\hline 要 & $\bar{s}$ \\
\hline 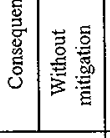 & 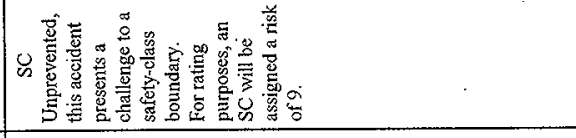 \\
\hline 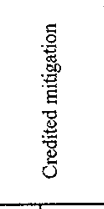 & 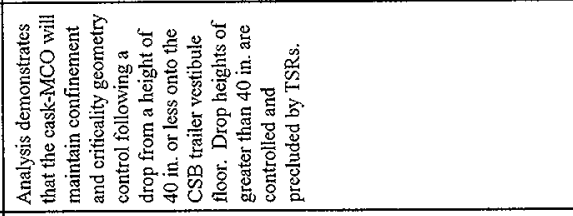 \\
\hline 焉惫 & is \\
\hline 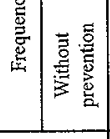 & 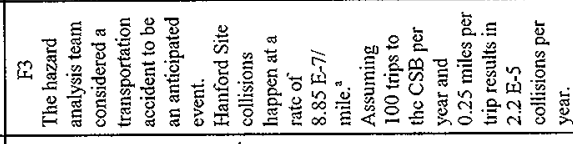 \\
\hline 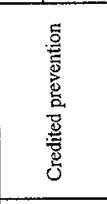 & 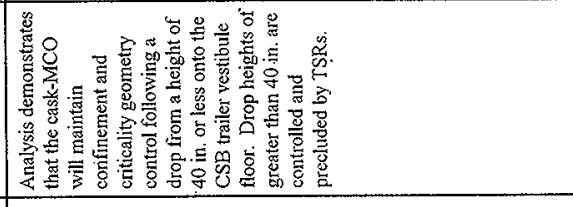 \\
\hline 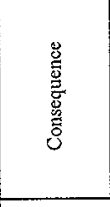 & 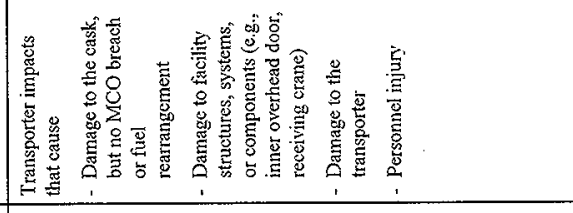 \\
\hline 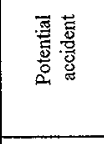 & 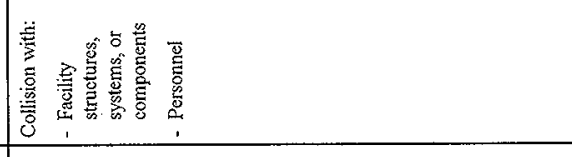 \\
\hline 煎 & 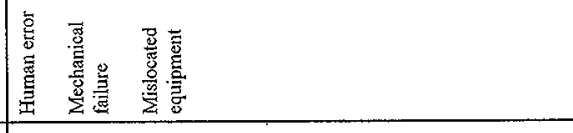 \\
\hline 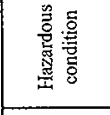 & 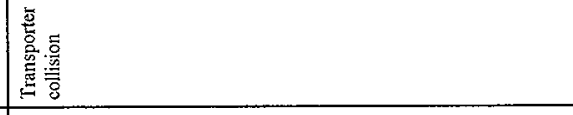 \\
\hline 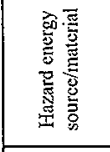 & 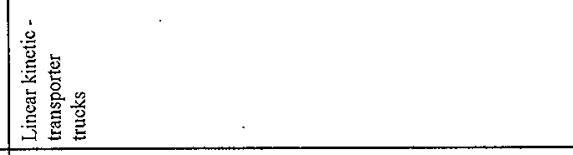 \\
\hline 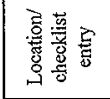 & 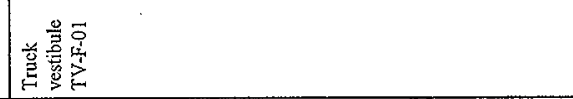 \\
\hline
\end{tabular}


HNF-SD-SNF-HIE-001 REV 1

\begin{tabular}{|c|c|c|}
\hline 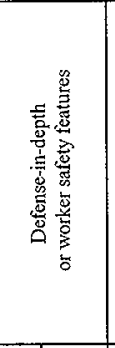 & 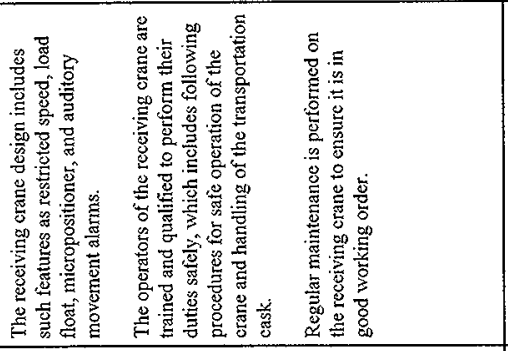 & 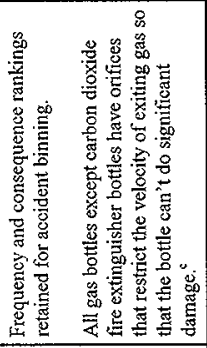 \\
\hline 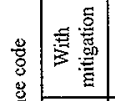 & $\bar{\infty}$ & : \\
\hline 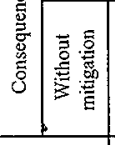 & 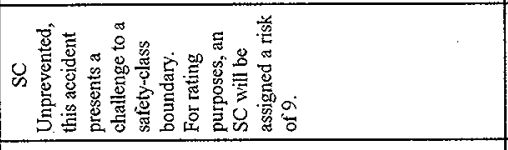 & $\tilde{\Sigma}$ \\
\hline 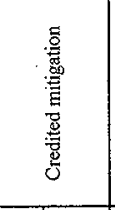 & 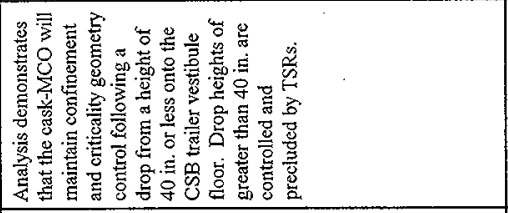 & 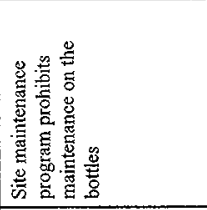 \\
\hline 尊 & 요 & i \\
\hline 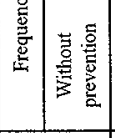 & 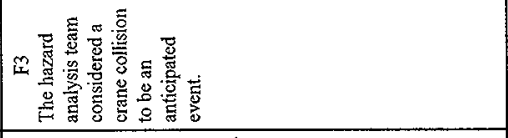 & N \\
\hline 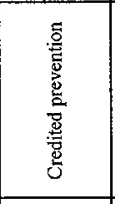 & 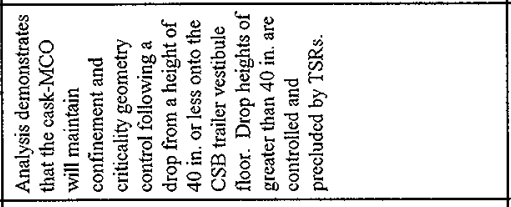 & 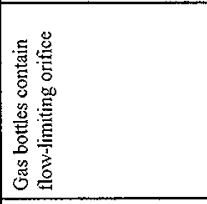 \\
\hline 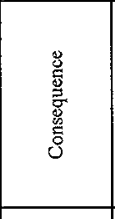 & 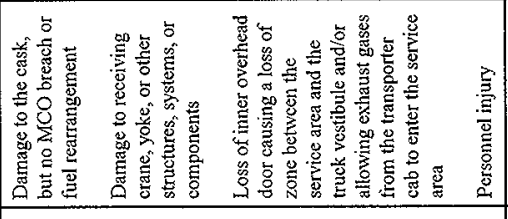 & 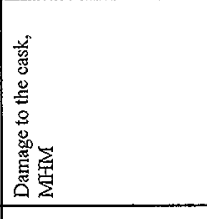 \\
\hline 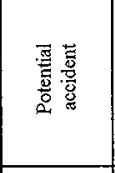 & 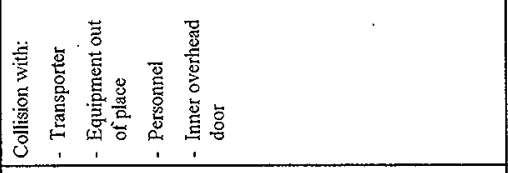 & 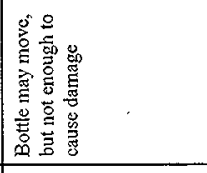 \\
\hline $\begin{array}{l}\text { 亗 } \\
\widetilde{J}\end{array}$ & 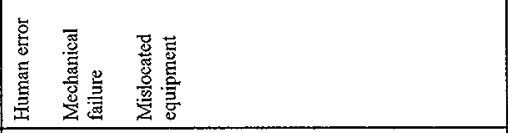 & 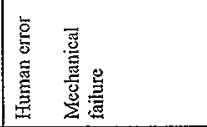 \\
\hline 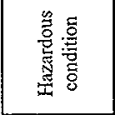 & 这 & 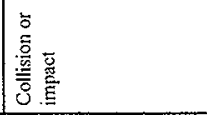 \\
\hline 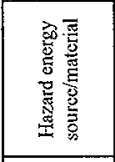 & 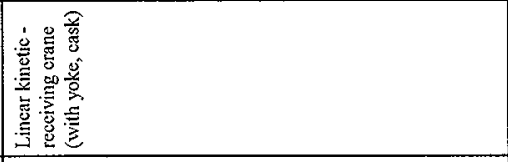 & 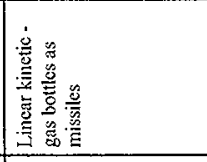 \\
\hline 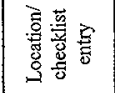 & 总令 & 总总早 \\
\hline
\end{tabular}


HNF-SD-SNF-HIE-00I REV 1

\begin{tabular}{|c|c|c|}
\hline 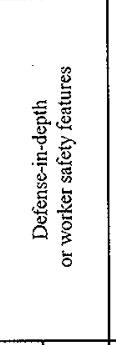 & 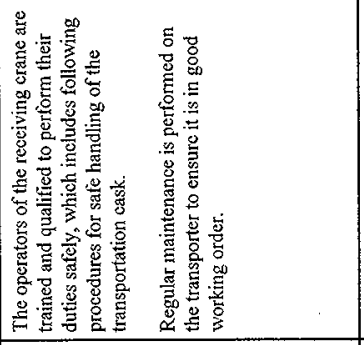 & 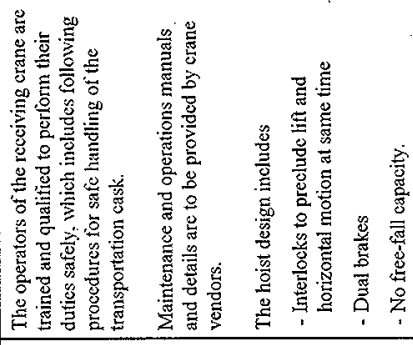 \\
\hline 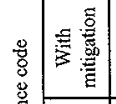 & $\vec{\omega}$ & $\bar{\omega}$ \\
\hline 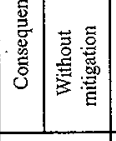 & 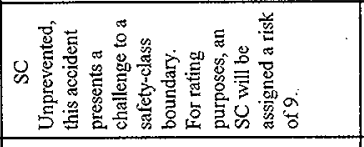 & 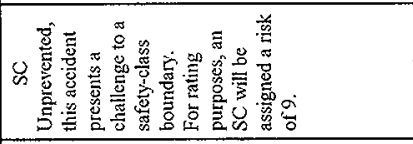 \\
\hline 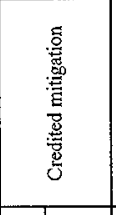 & 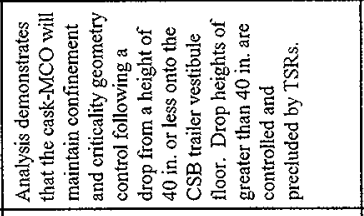 & 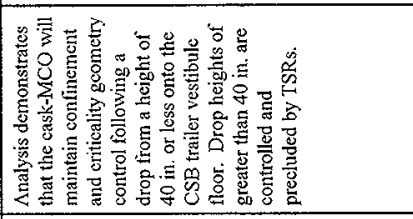 \\
\hline 卷咅 & 屈 & 운 \\
\hline 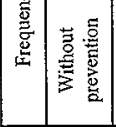 & $m$ & 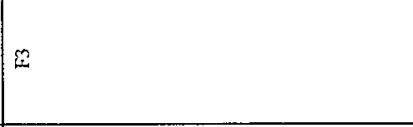 \\
\hline 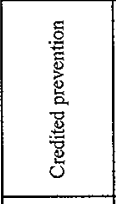 & 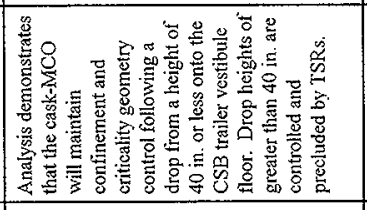 & 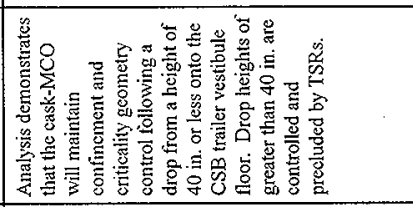 \\
\hline 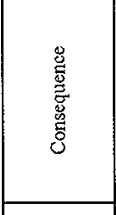 & 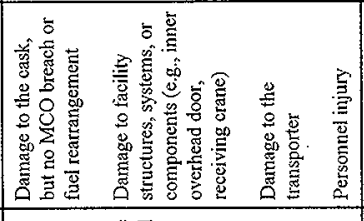 & 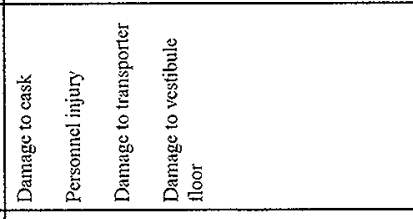 \\
\hline 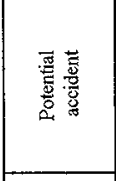 & 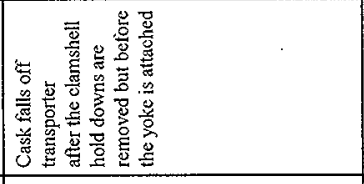 & 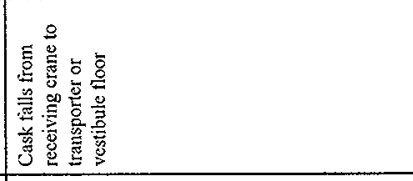 \\
\hline 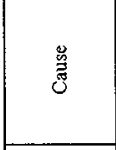 & 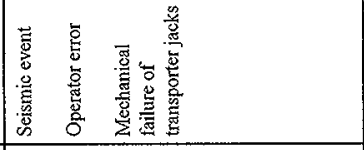 & 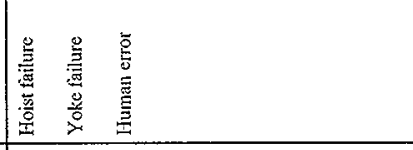 \\
\hline 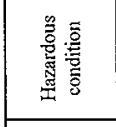 & 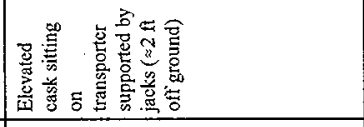 & 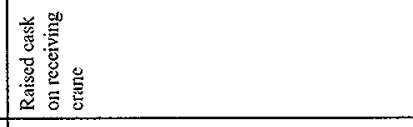 \\
\hline 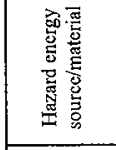 & 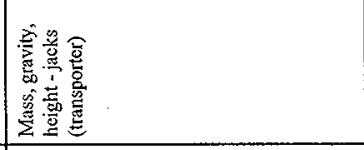 & 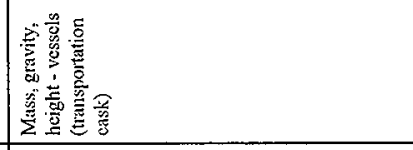 \\
\hline 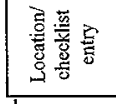 & 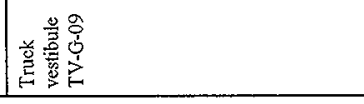 & 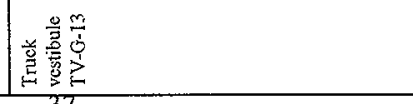 \\
\hline
\end{tabular}


HNF-SD-SNF-HIE-001 REV 1

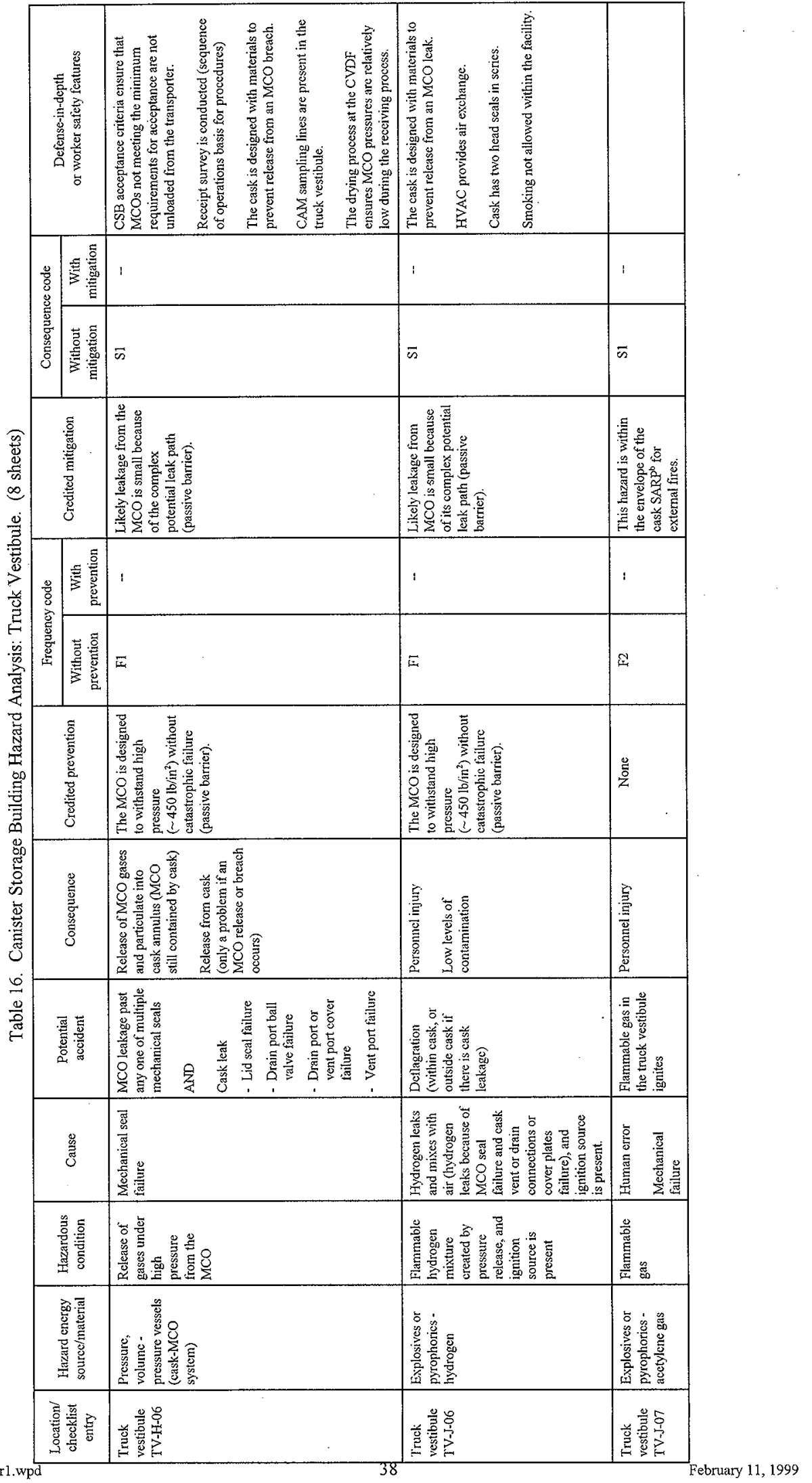




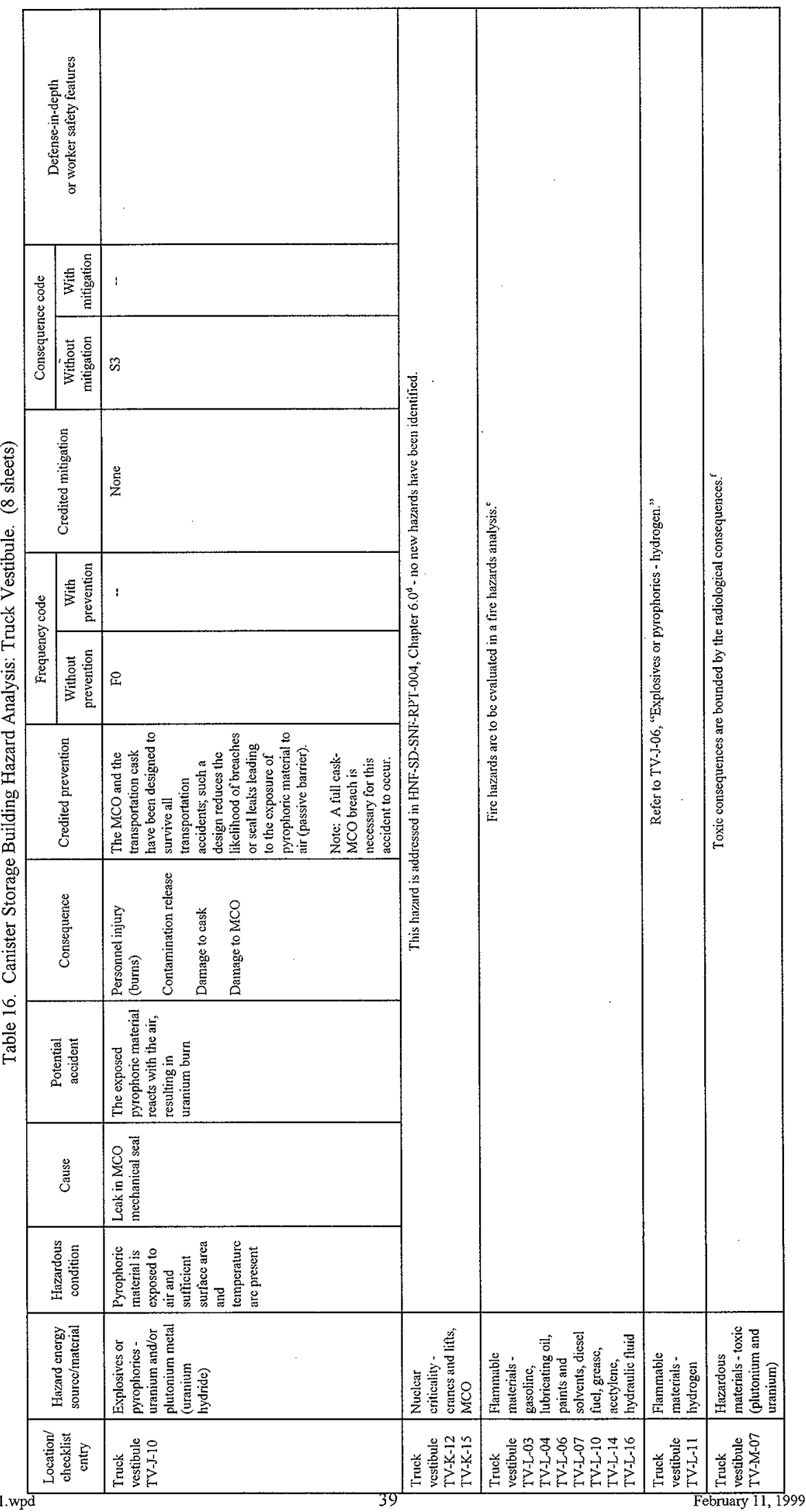


HNF-SD-SNF-HIE-001 REV 1

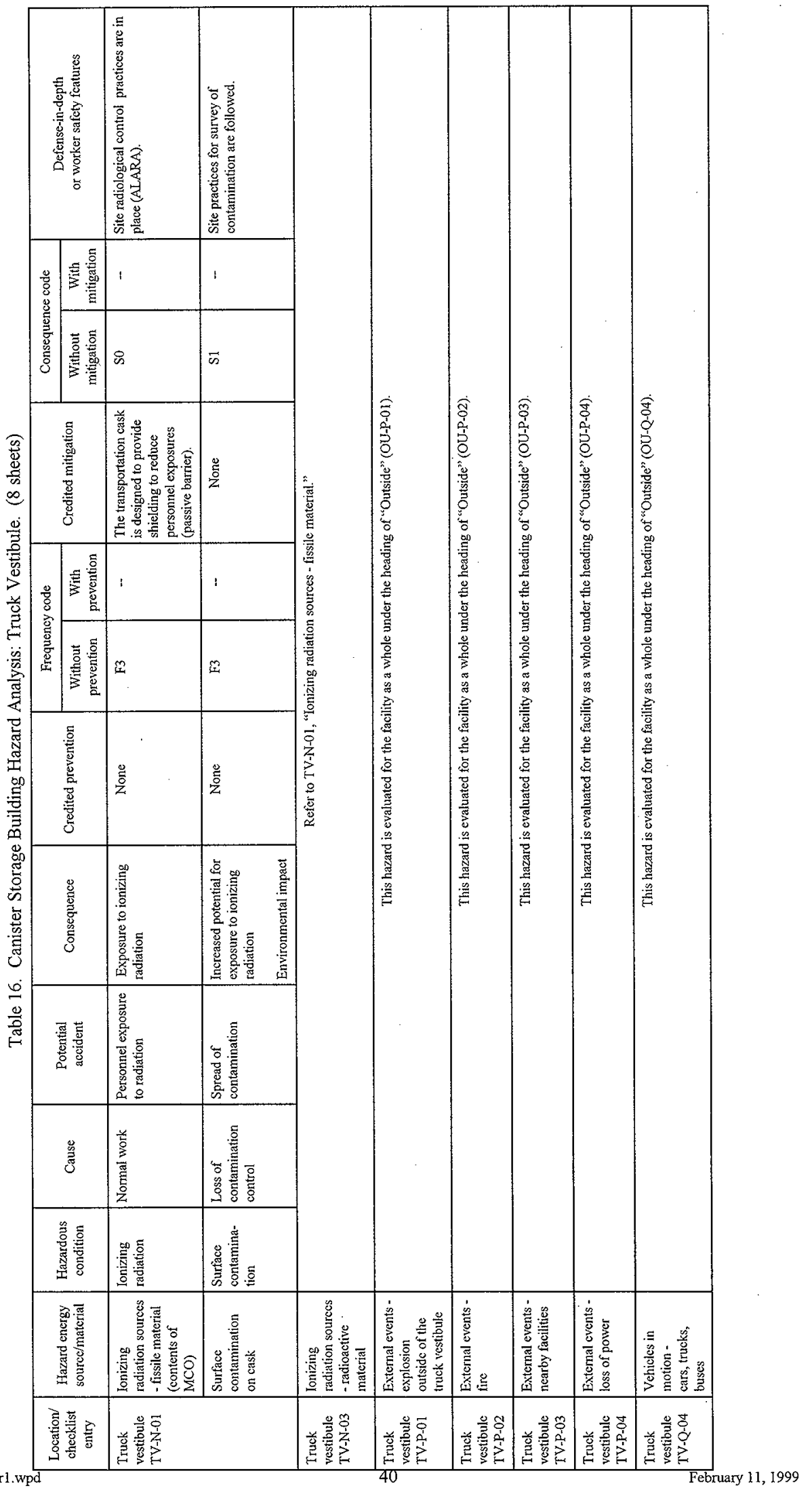




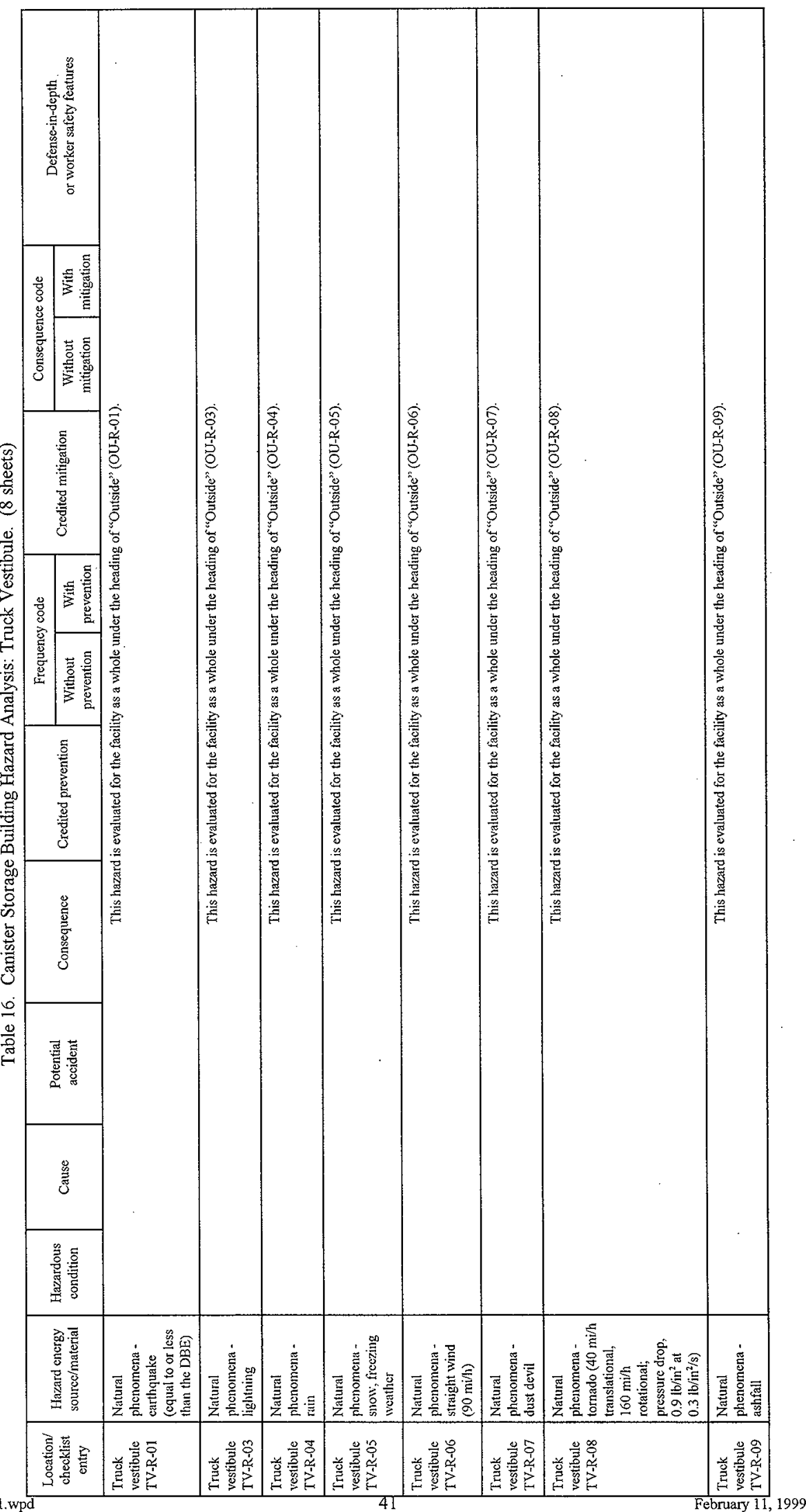




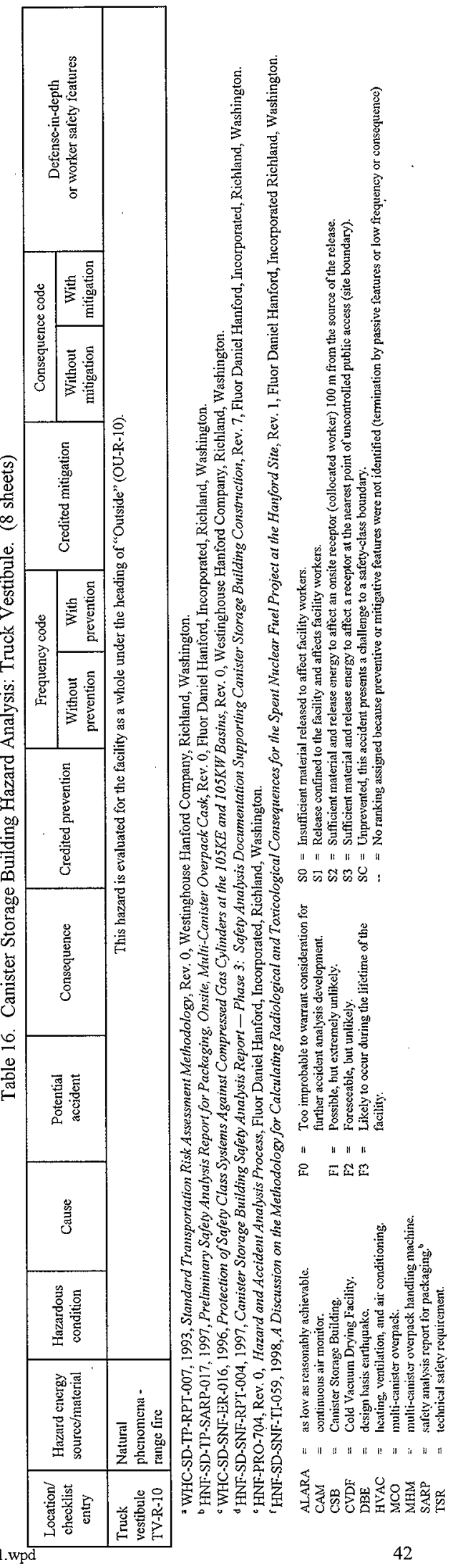


HNF-SD-SNF-HIE-001 REV 1

\begin{tabular}{|c|c|c|}
\hline 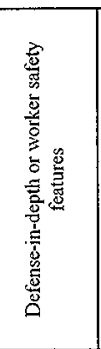 & 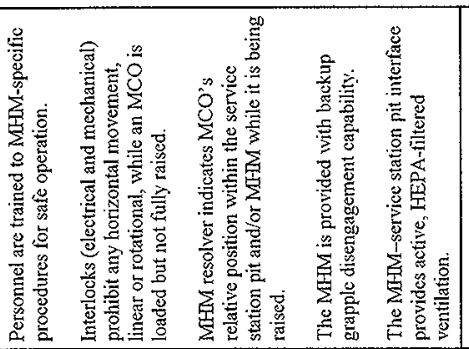 & 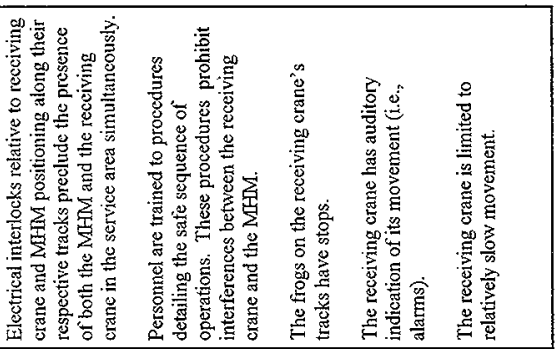 \\
\hline 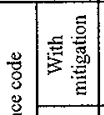 & ( & 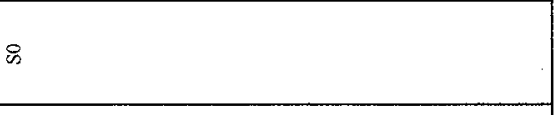 \\
\hline | & 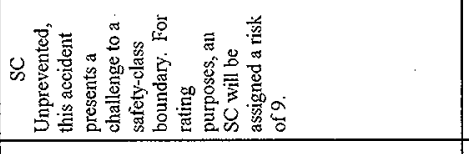 & 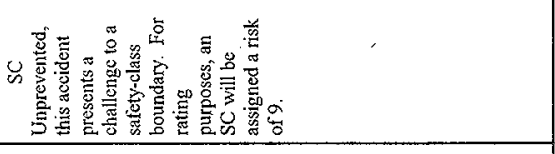 \\
\hline 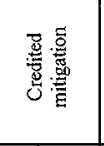 & $\stackrel{0}{\bar{z}}$ & 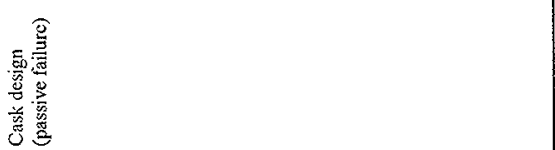 \\
\hline 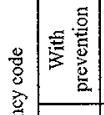 & Fis & $\Xi$ \\
\hline 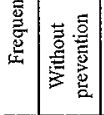 & 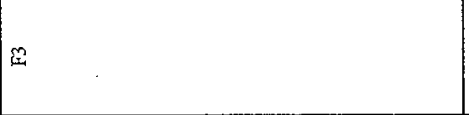 & 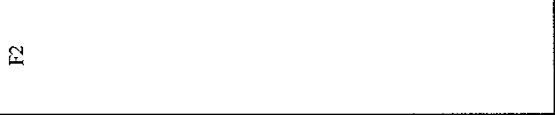 \\
\hline 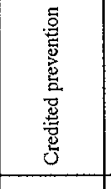 & 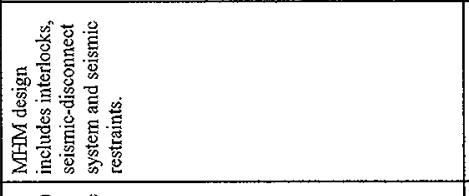 & 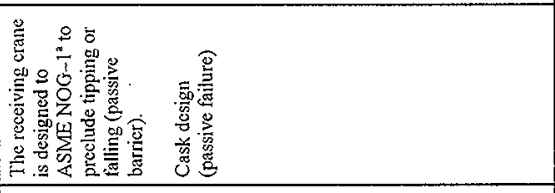 \\
\hline 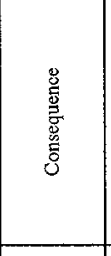 & 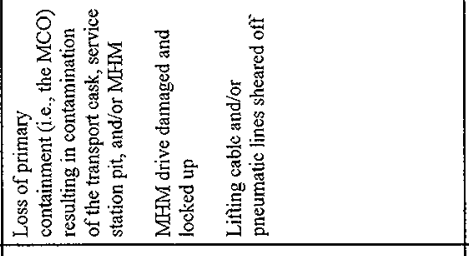 & 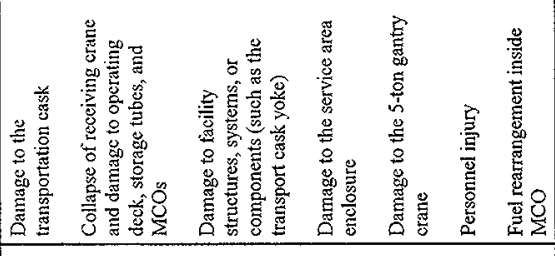 \\
\hline 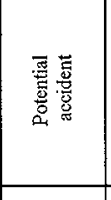 & 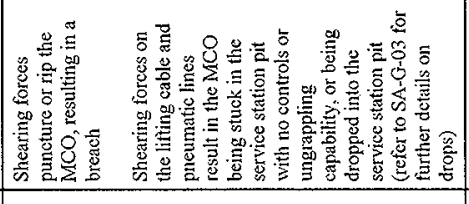 & 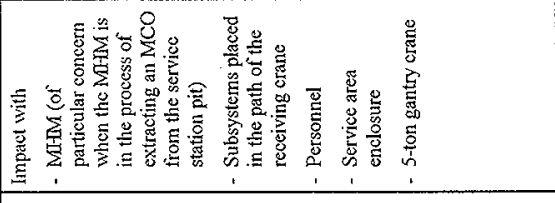 \\
\hline 愛 & 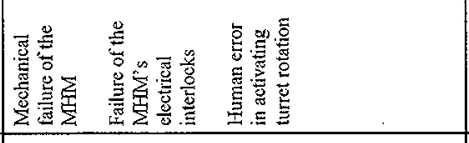 & 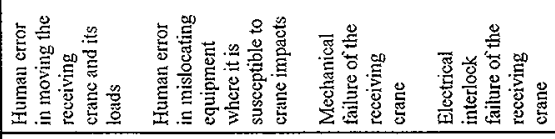 \\
\hline 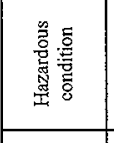 & 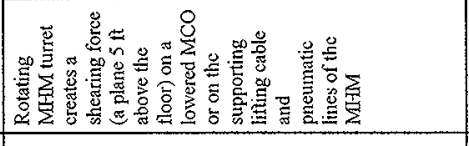 & 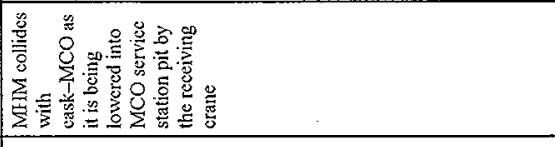 \\
\hline 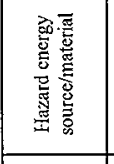 & 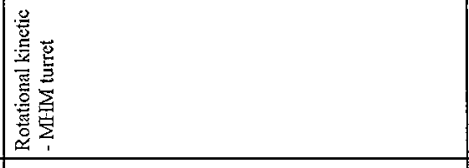 & 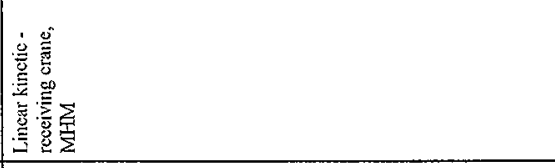 \\
\hline 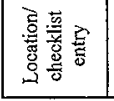 & 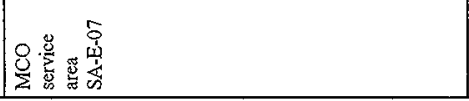 & 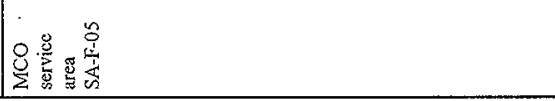 \\
\hline
\end{tabular}


HNF-SD-SNF-HIE-001 REV 1

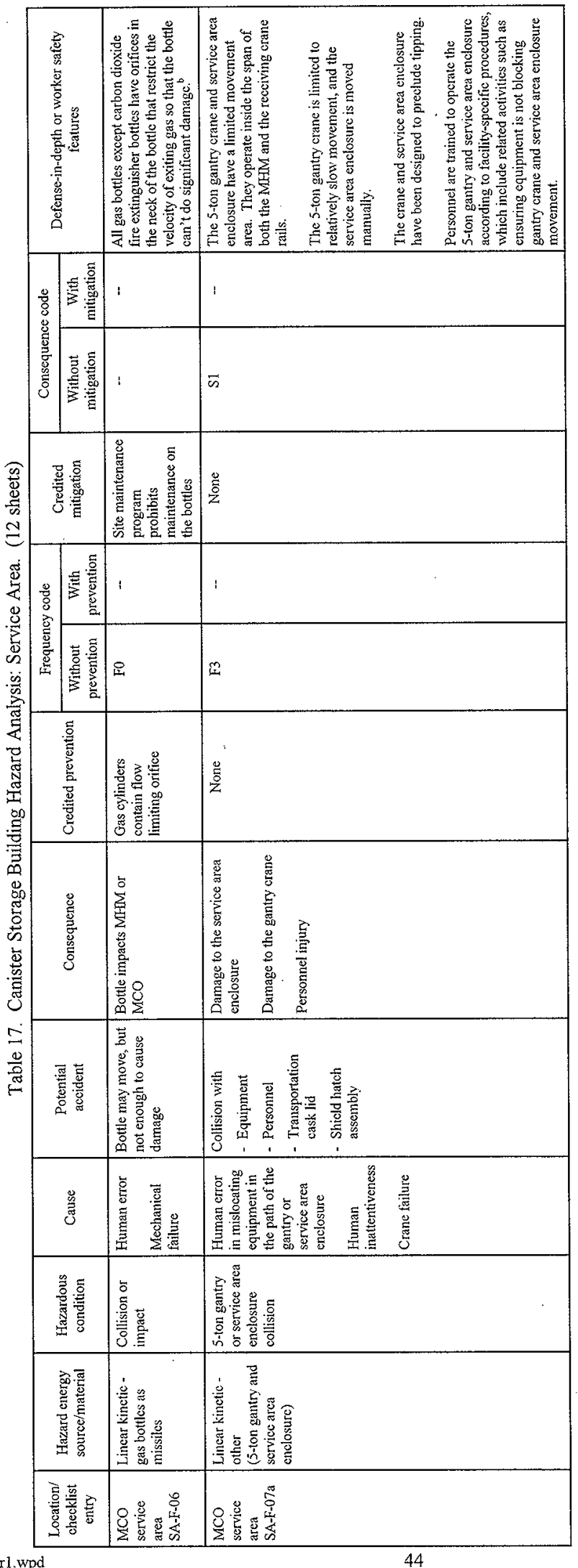


HNF-SD-SNF-HIE-001 REV 1

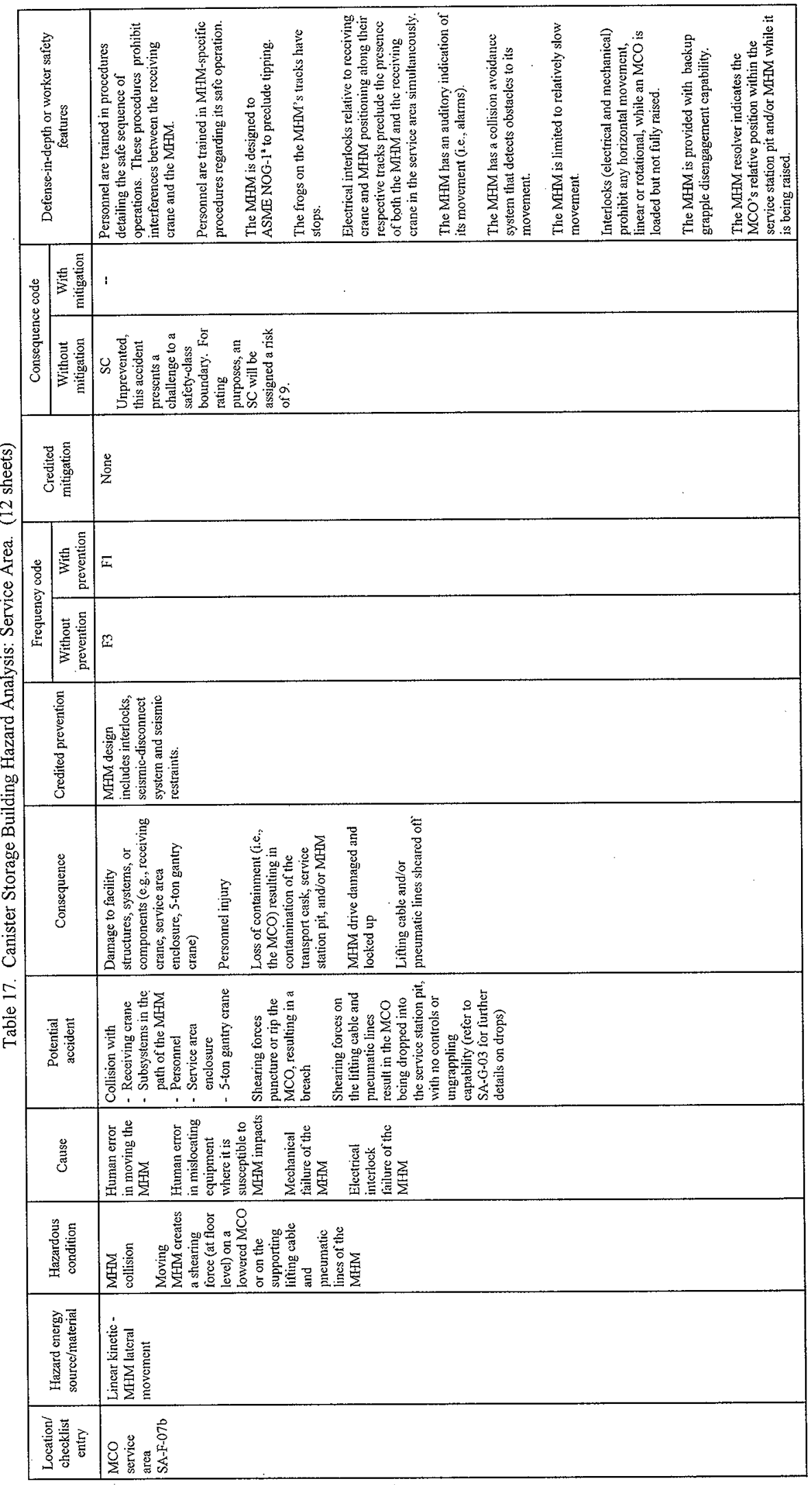


HNF-SD-SNF-HIE-00I REV 1

\begin{tabular}{|c|c|}
\hline 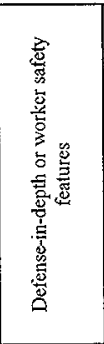 & 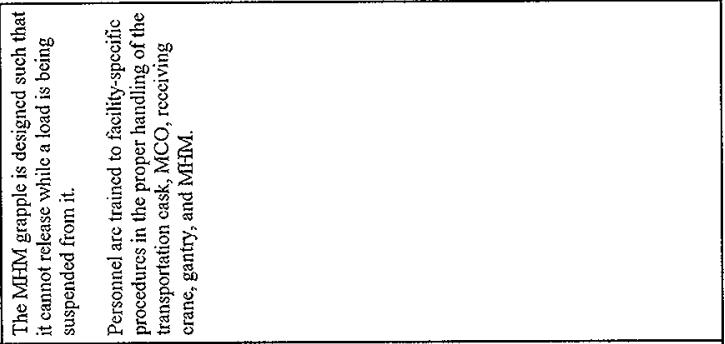 \\
\hline 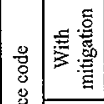 & : \\
\hline 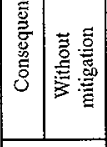 & 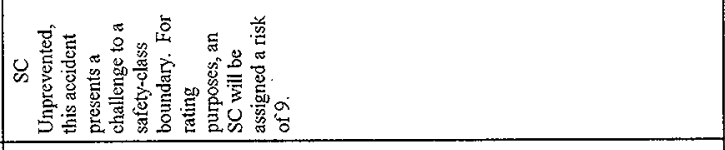 \\
\hline 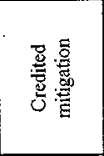 & 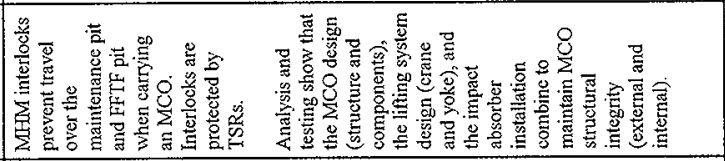 \\
\hline 营营 & 용 \\
\hline 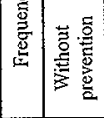 & 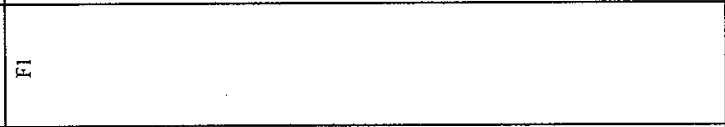 \\
\hline 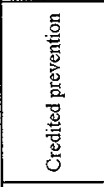 & 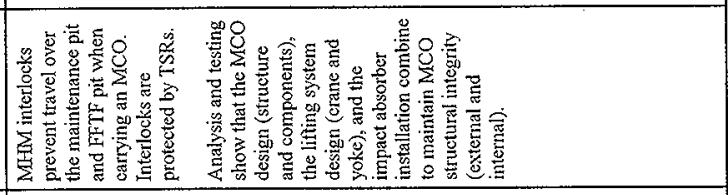 \\
\hline 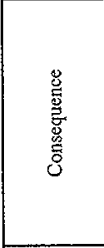 & 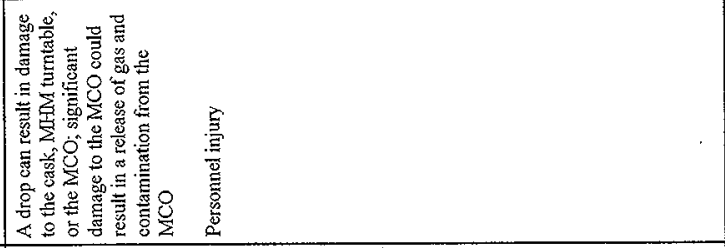 \\
\hline 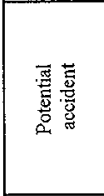 & 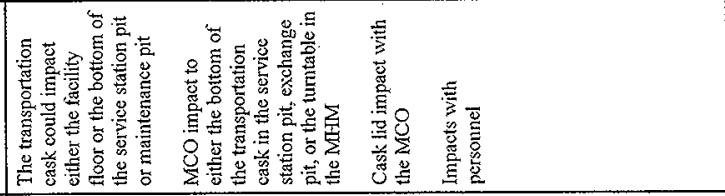 \\
\hline $\begin{array}{l}\text { o. } \\
\text { 危 }\end{array}$ & 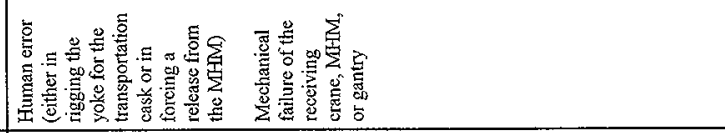 \\
\hline 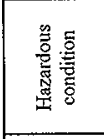 & 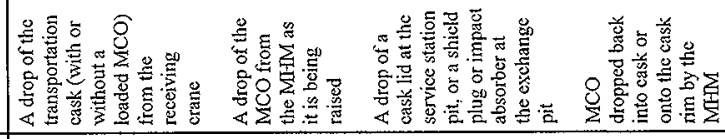 \\
\hline 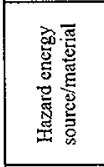 & 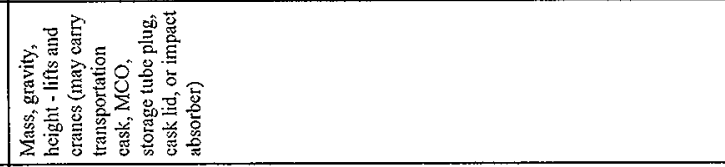 \\
\hline 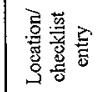 & $8 \underbrace{\circ}$ \\
\hline
\end{tabular}




\begin{tabular}{|c|c|}
\hline 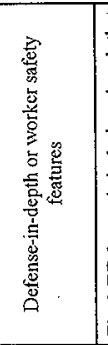 & 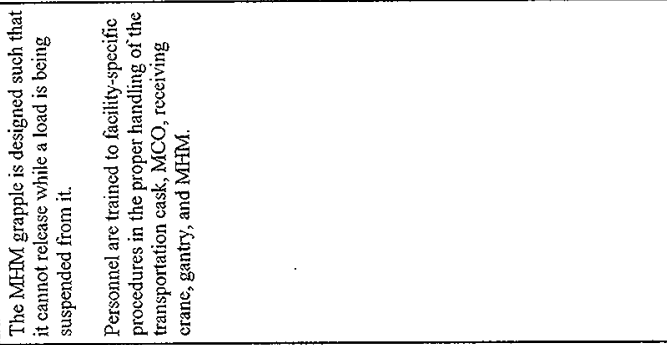 \\
\hline 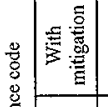 & 1 \\
\hline 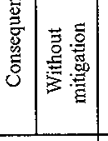 & 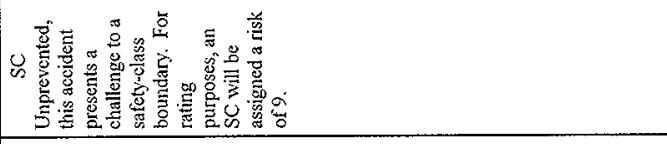 \\
\hline 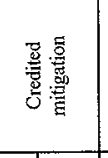 & 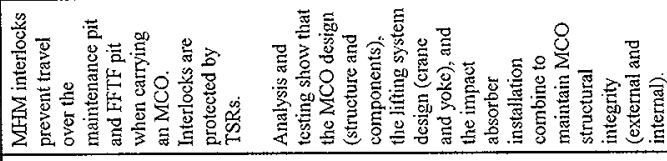 \\
\hline 䓪 & 웅 \\
\hline \begin{tabular}{|l|l|} 
\\
\end{tabular} & $\bar{x}$ \\
\hline 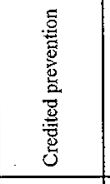 & 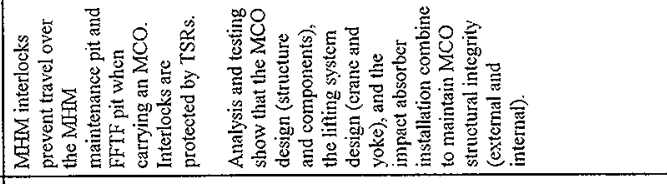 \\
\hline 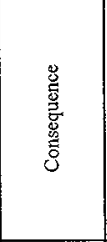 & 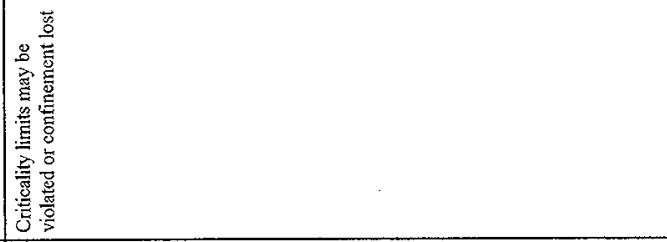 \\
\hline 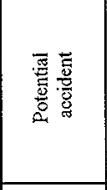 & 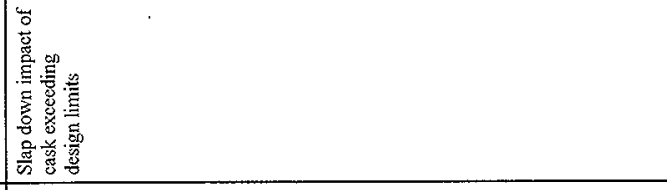 \\
\hline 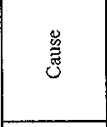 & 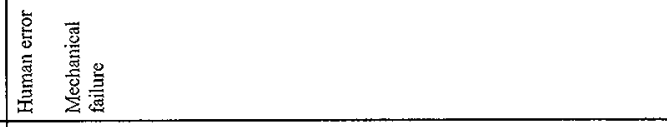 \\
\hline 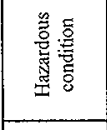 & 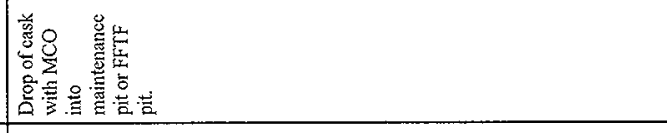 \\
\hline 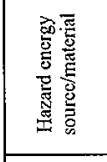 & 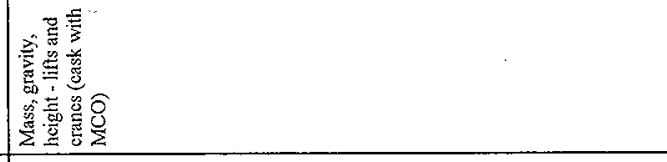 \\
\hline 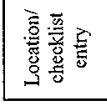 & 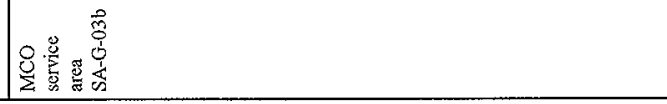 \\
\hline
\end{tabular}




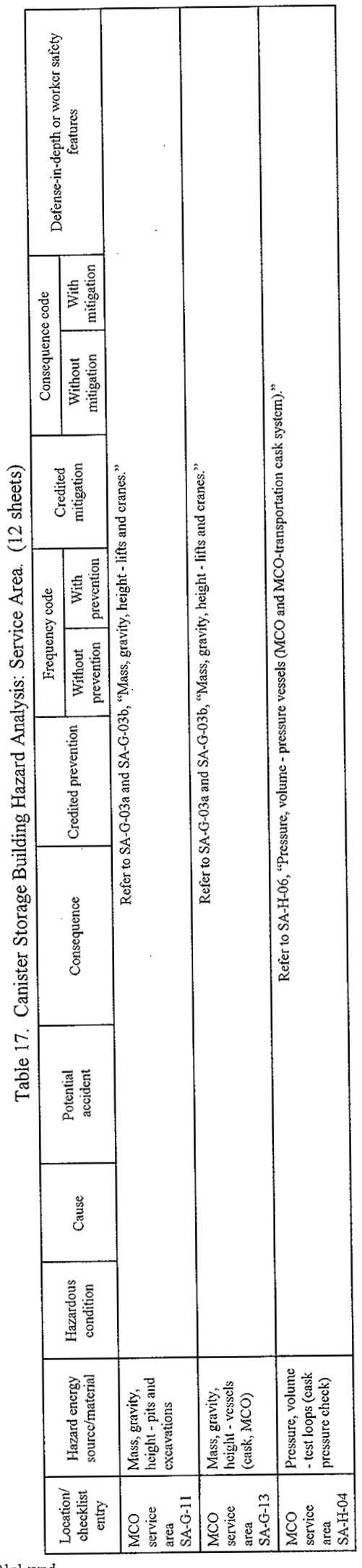


HNF-SD-SNF-HIE-001 REV 1

\begin{tabular}{|c|c|}
\hline 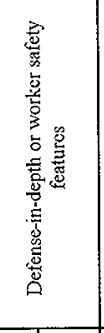 & 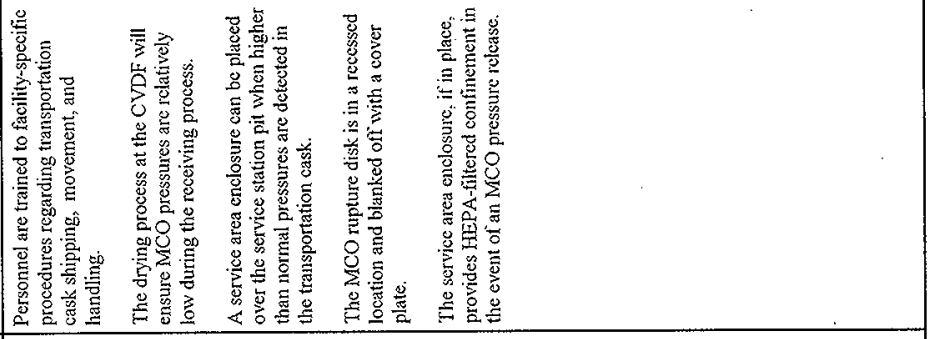 \\
\hline 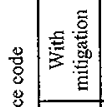 & 1 \\
\hline 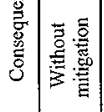 & कs \\
\hline 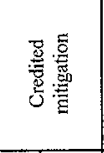 & 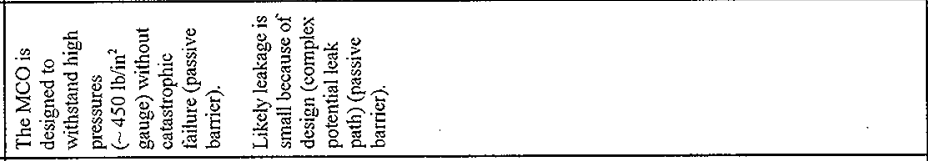 \\
\hline 尊 & 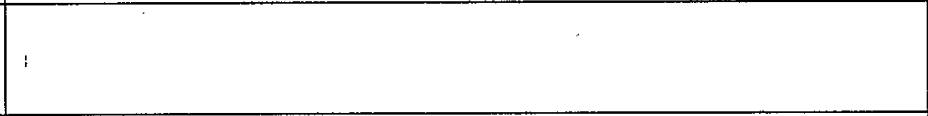 \\
\hline 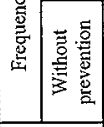 & 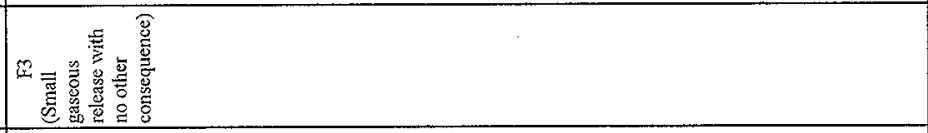 \\
\hline 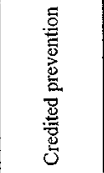 & 蒙 \\
\hline 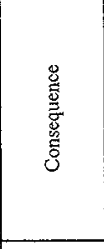 & 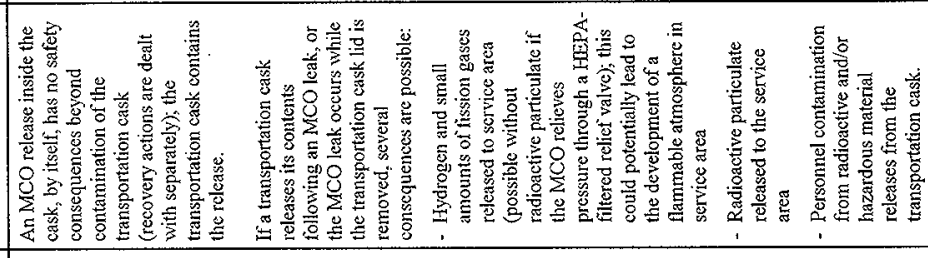 \\
\hline 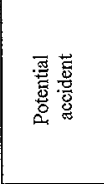 & 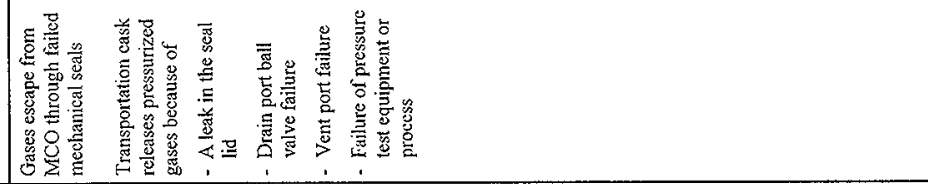 \\
\hline 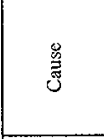 & 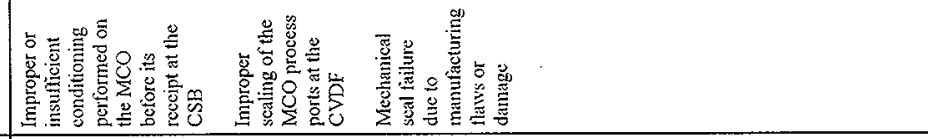 \\
\hline 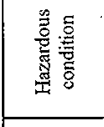 & 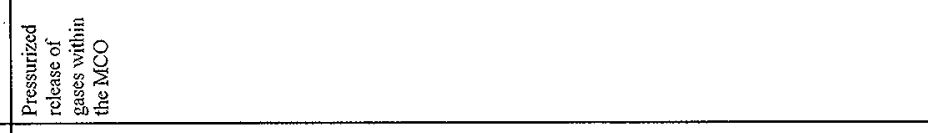 \\
\hline 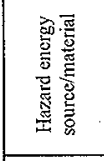 & 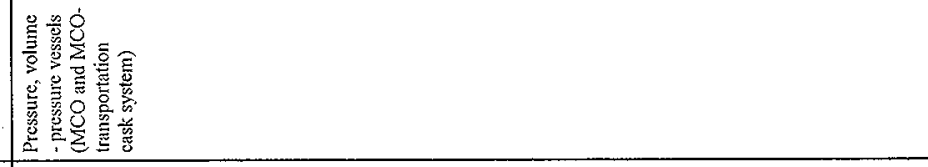 \\
\hline 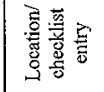 & 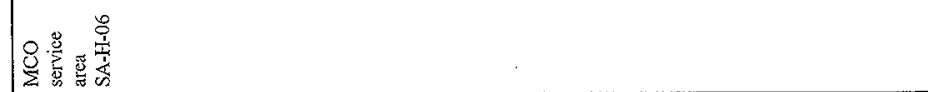 \\
\hline
\end{tabular}


HNF-SD-SNF-HIE-001 REV 1

\begin{tabular}{|c|c|c|c|}
\hline 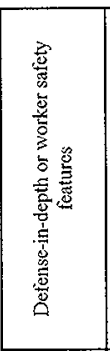 & 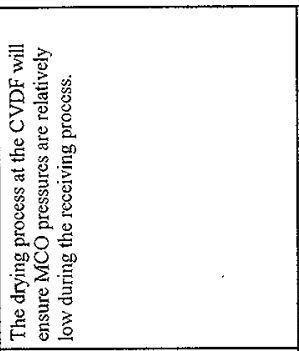 & & 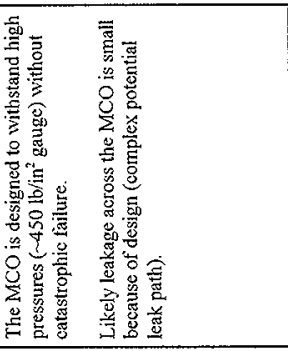 \\
\hline 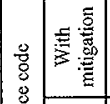 & 1 & $\bar{\omega}$ & 1 \\
\hline 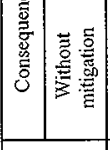 & 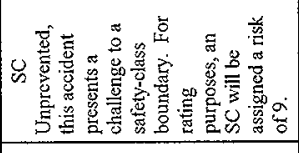 & $\approx$ & 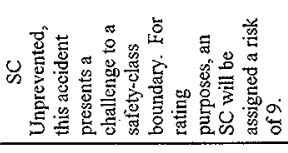 \\
\hline 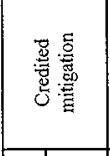 & 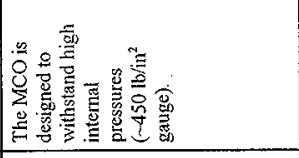 & 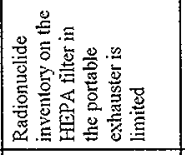 & $\stackrel{\frac{\mathscr{g}}{5}}{\frac{2}{2}}$ \\
\hline 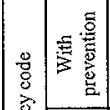 & : & $:$ & : \\
\hline 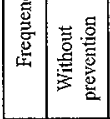 & $\cong$ & 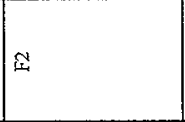 & $\Phi$ \\
\hline 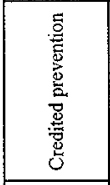 & 号 & 产 & 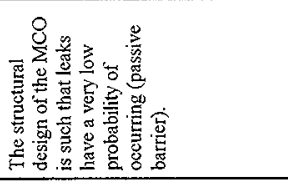 \\
\hline 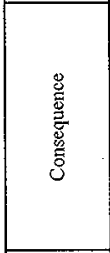 & 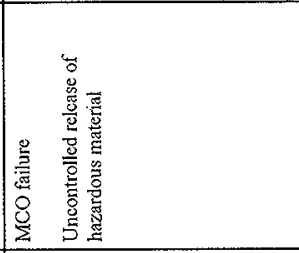 & 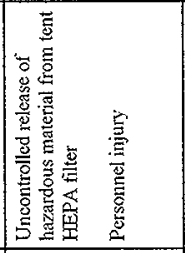 & 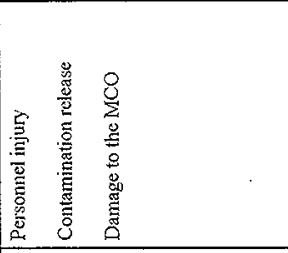 \\
\hline 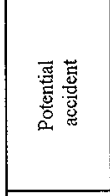 & 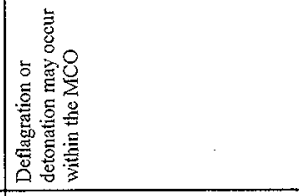 & 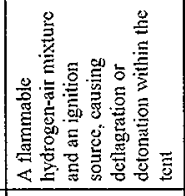 & 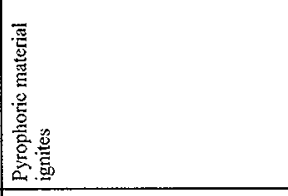 \\
\hline 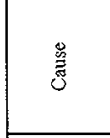 & 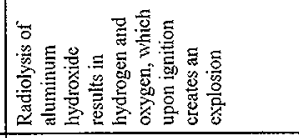 & 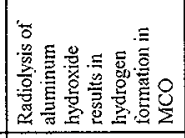 & 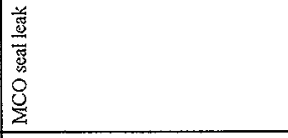 \\
\hline 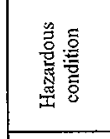 & 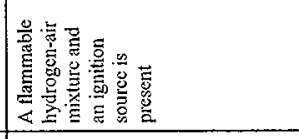 & 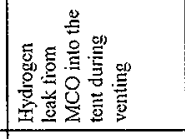 & 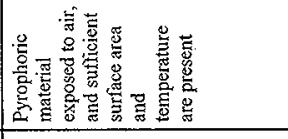 \\
\hline 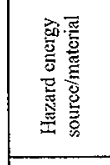 & 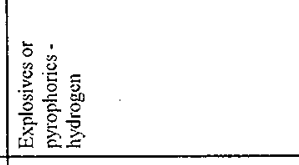 & 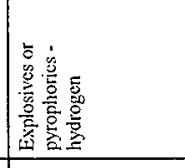 & 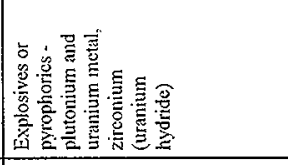 \\
\hline 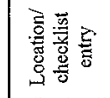 & 总总总离 & 总总总离 & 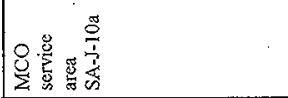 \\
\hline
\end{tabular}


HNF-SD-SNF-HIE-001 REV 1

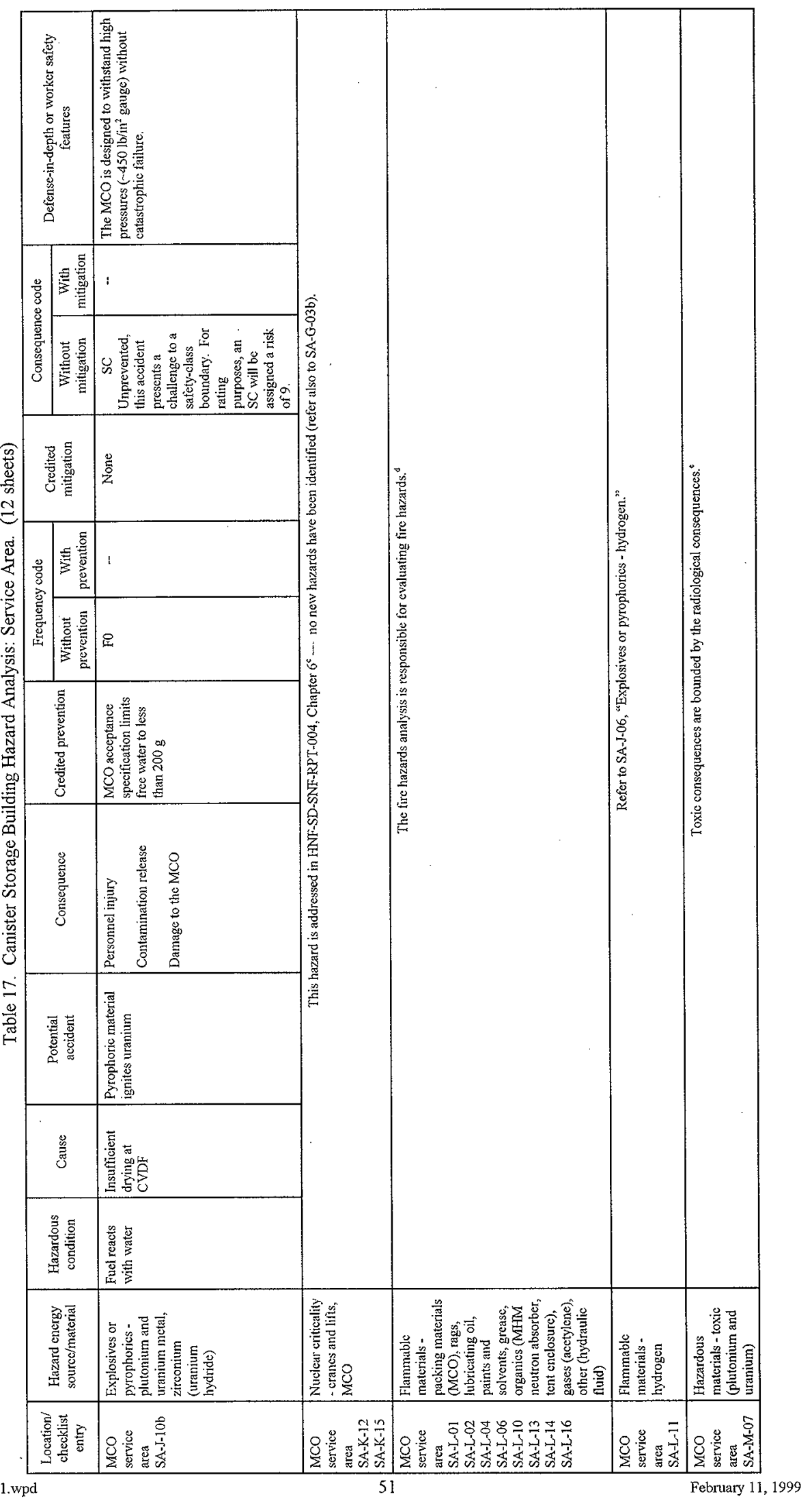


HNF-SD-SNF-HIE-001 REV 1

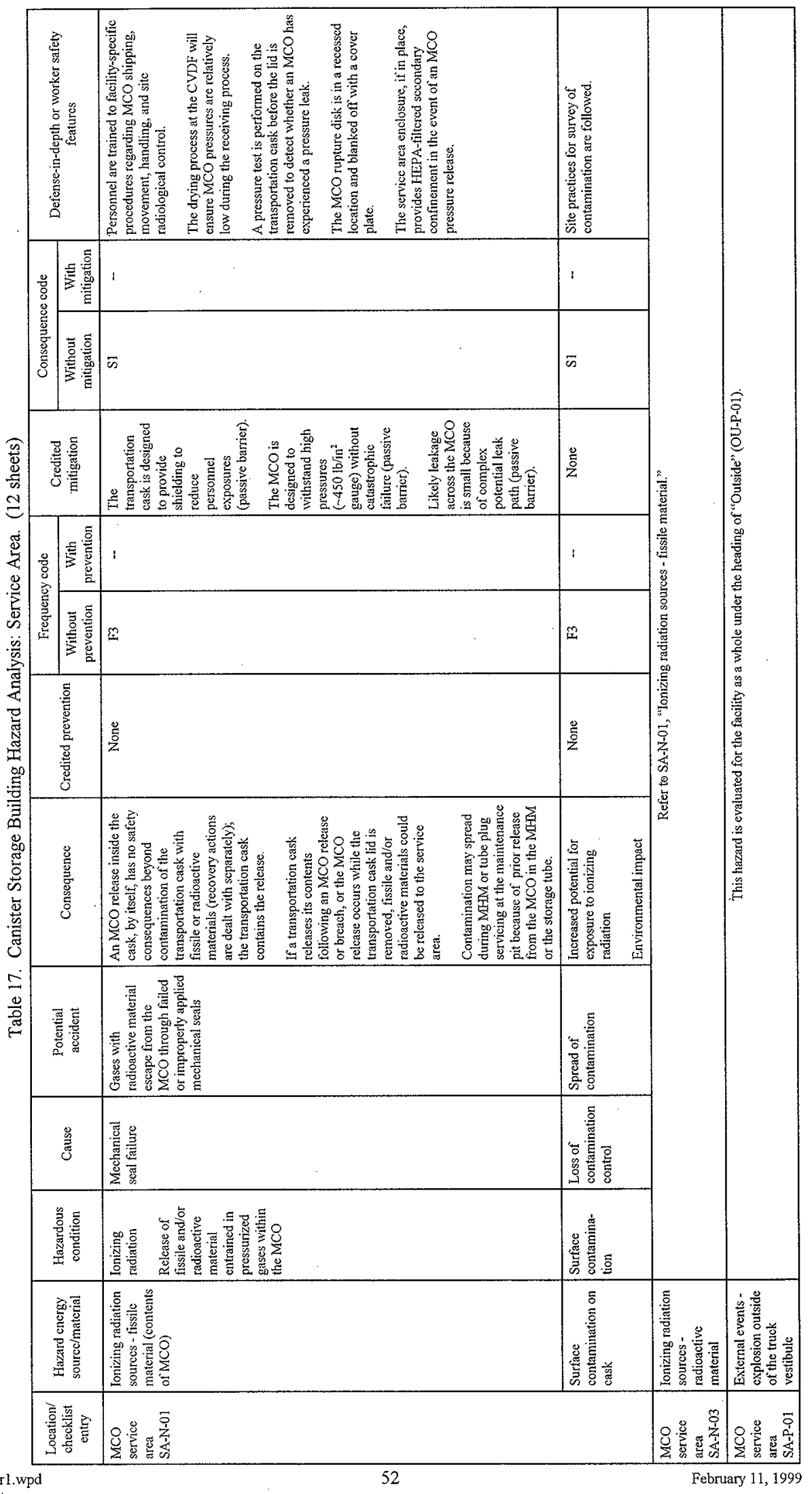




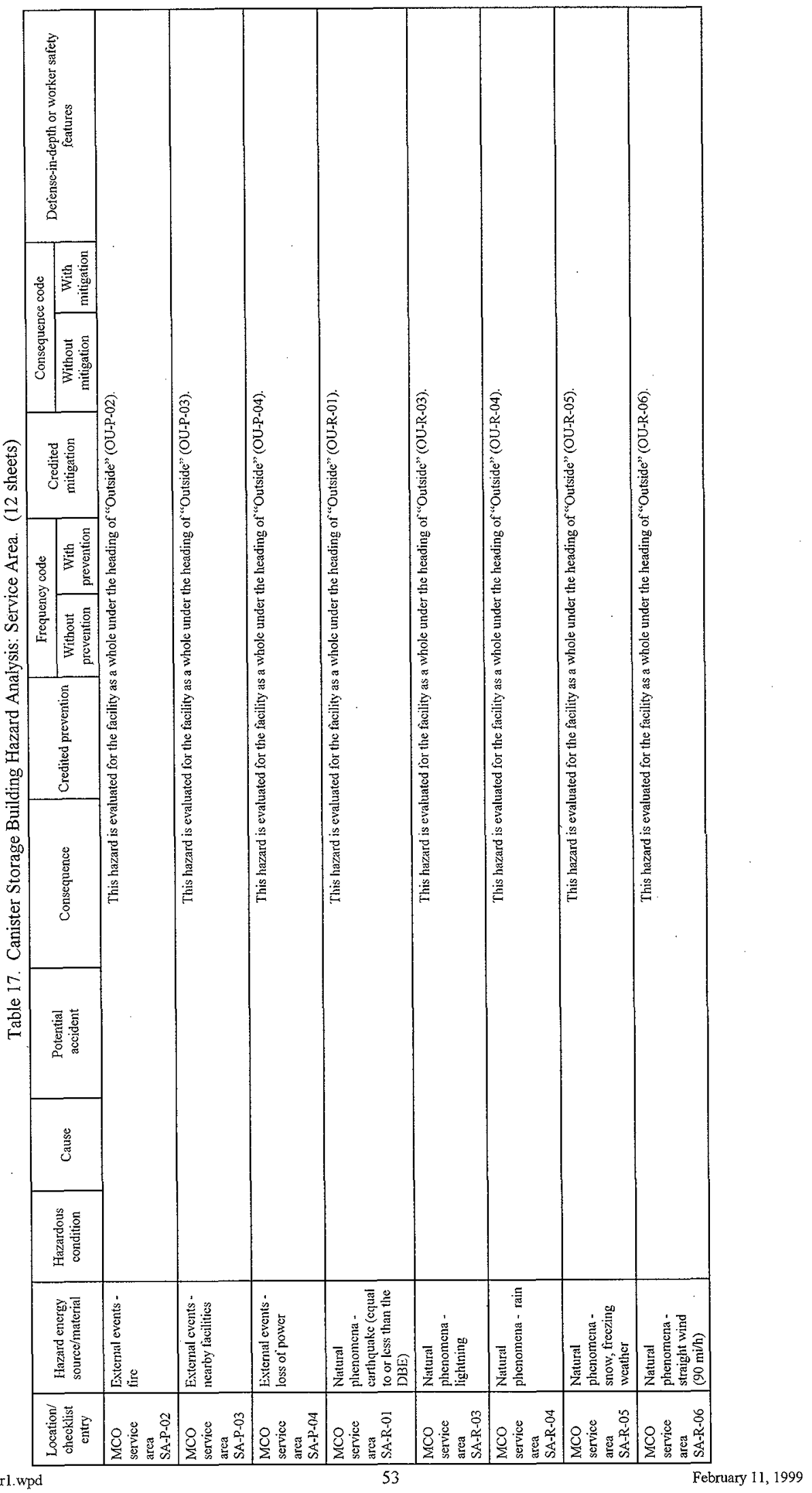


HNF-SD-SNF-HIE-001 REV 1

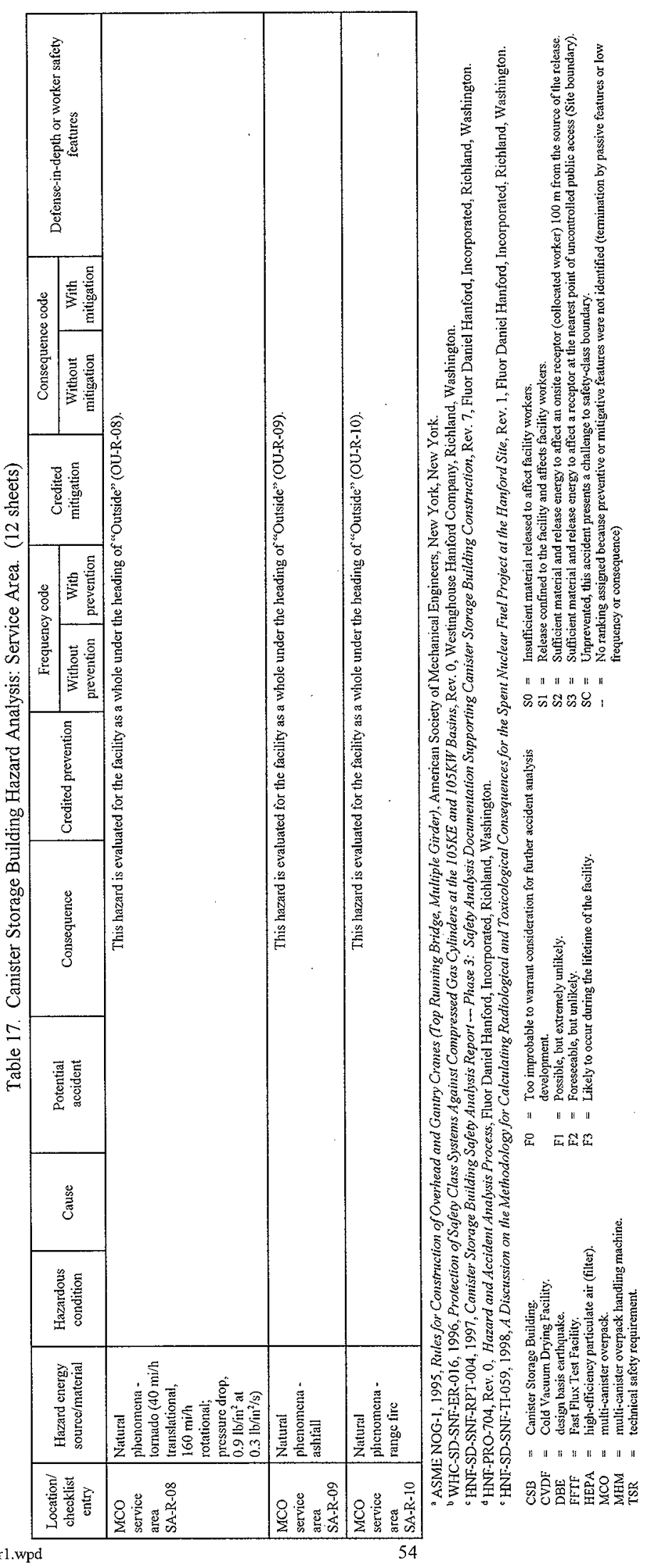

February 11, 1999 
HNE-SD-SNF-HIE-001 REV I

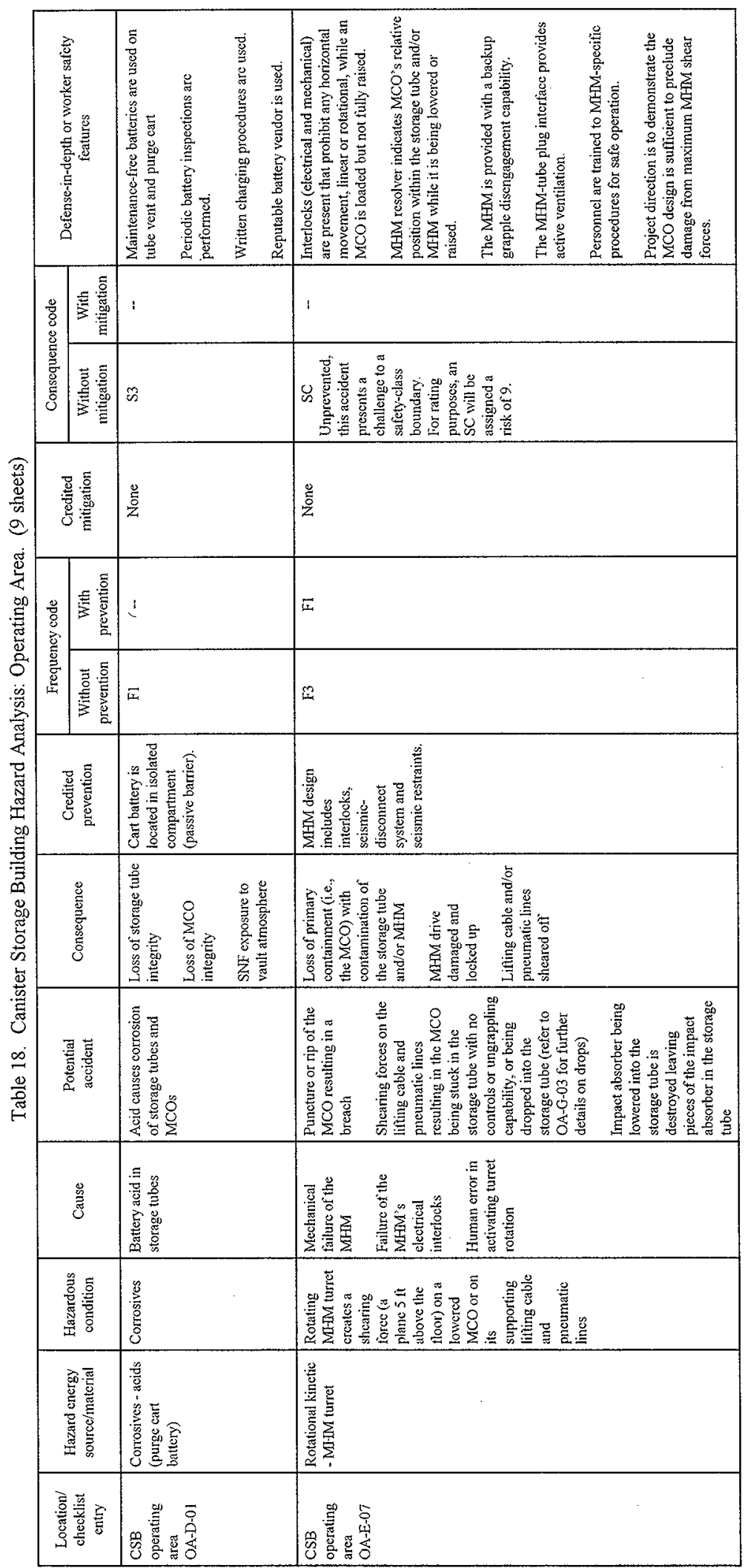


HNF-SD-SNF-HIE-001 REV 1

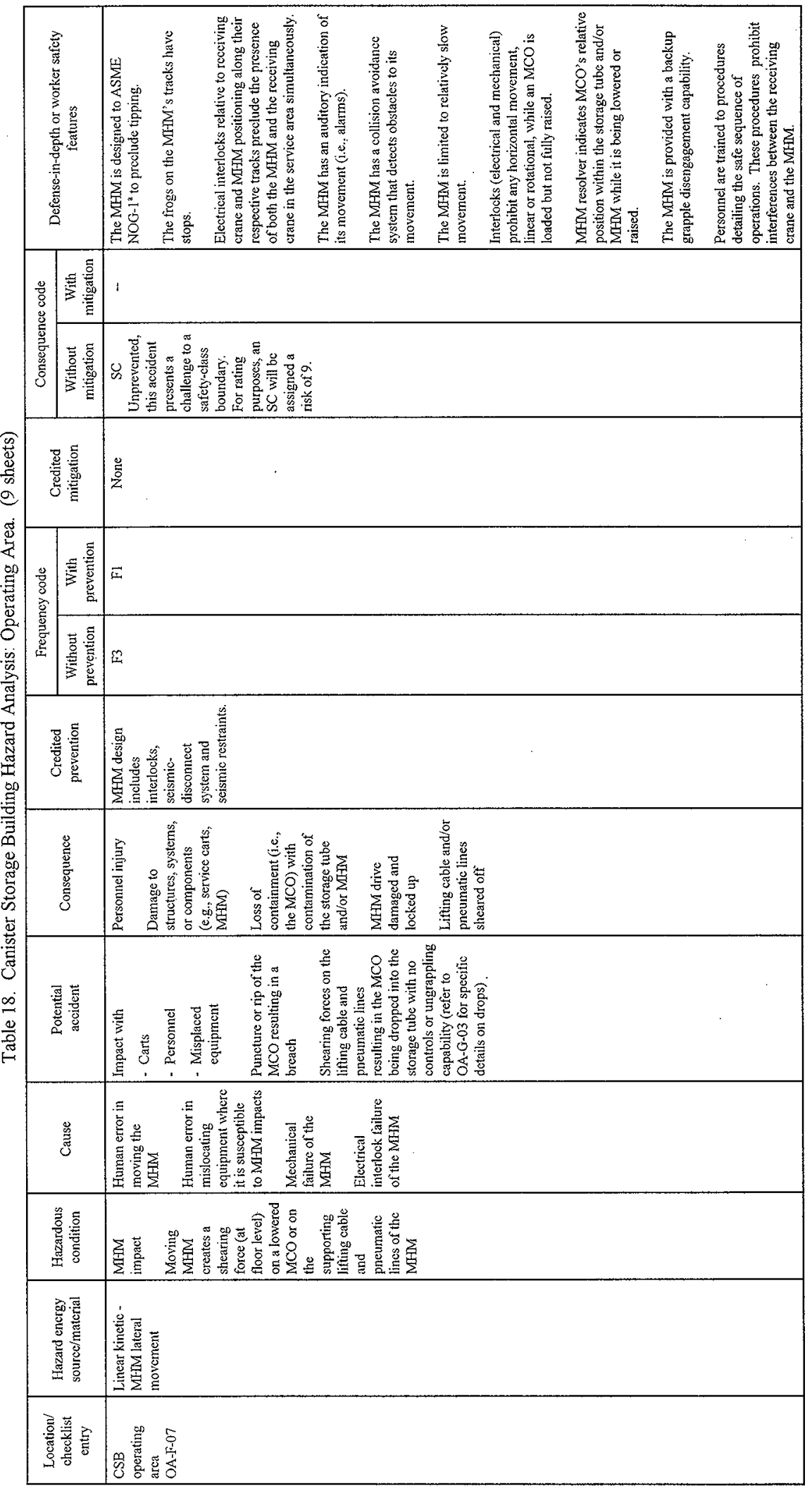


HNF-SD-SNF-HIE-001 REV 1

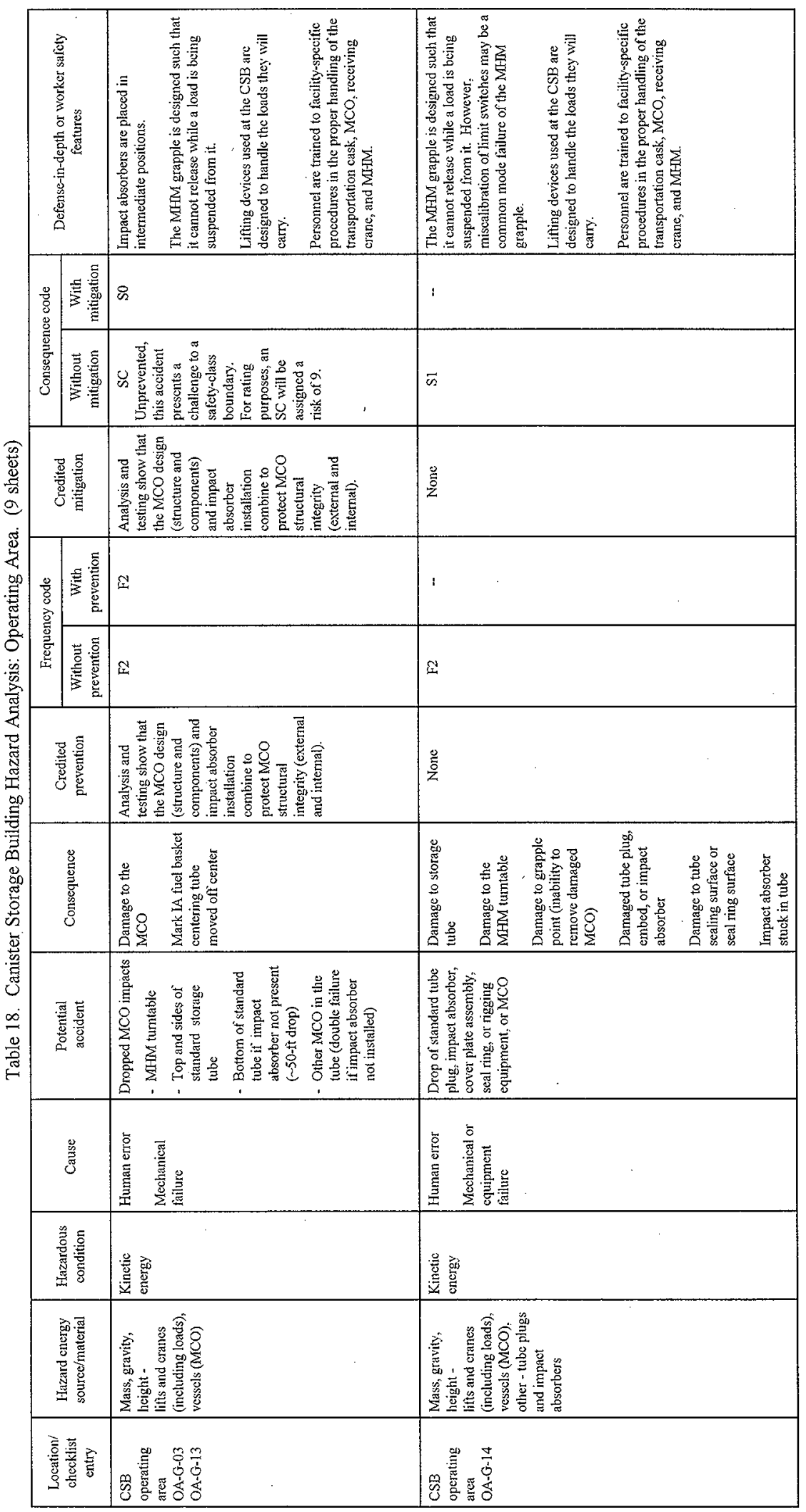




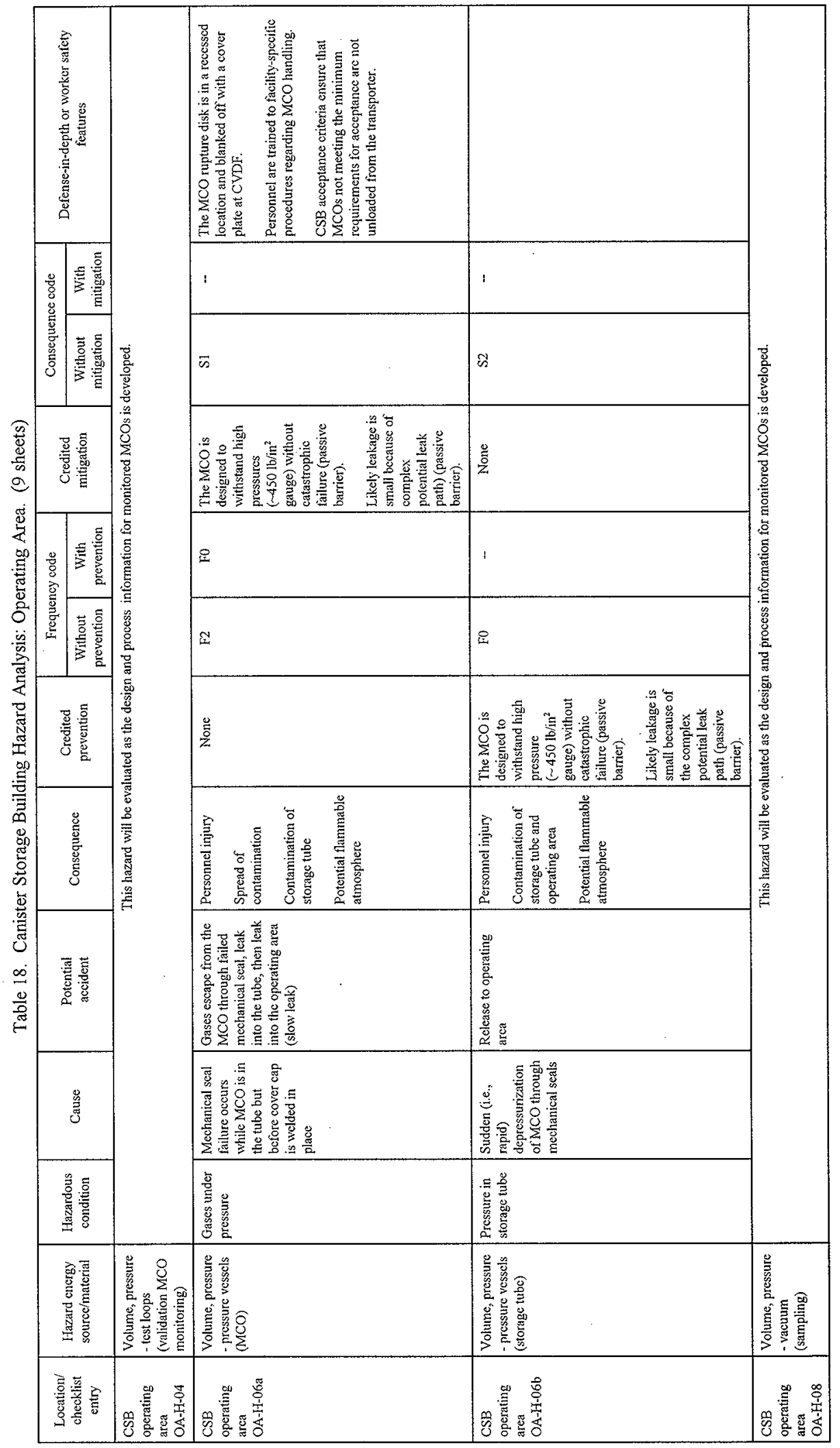




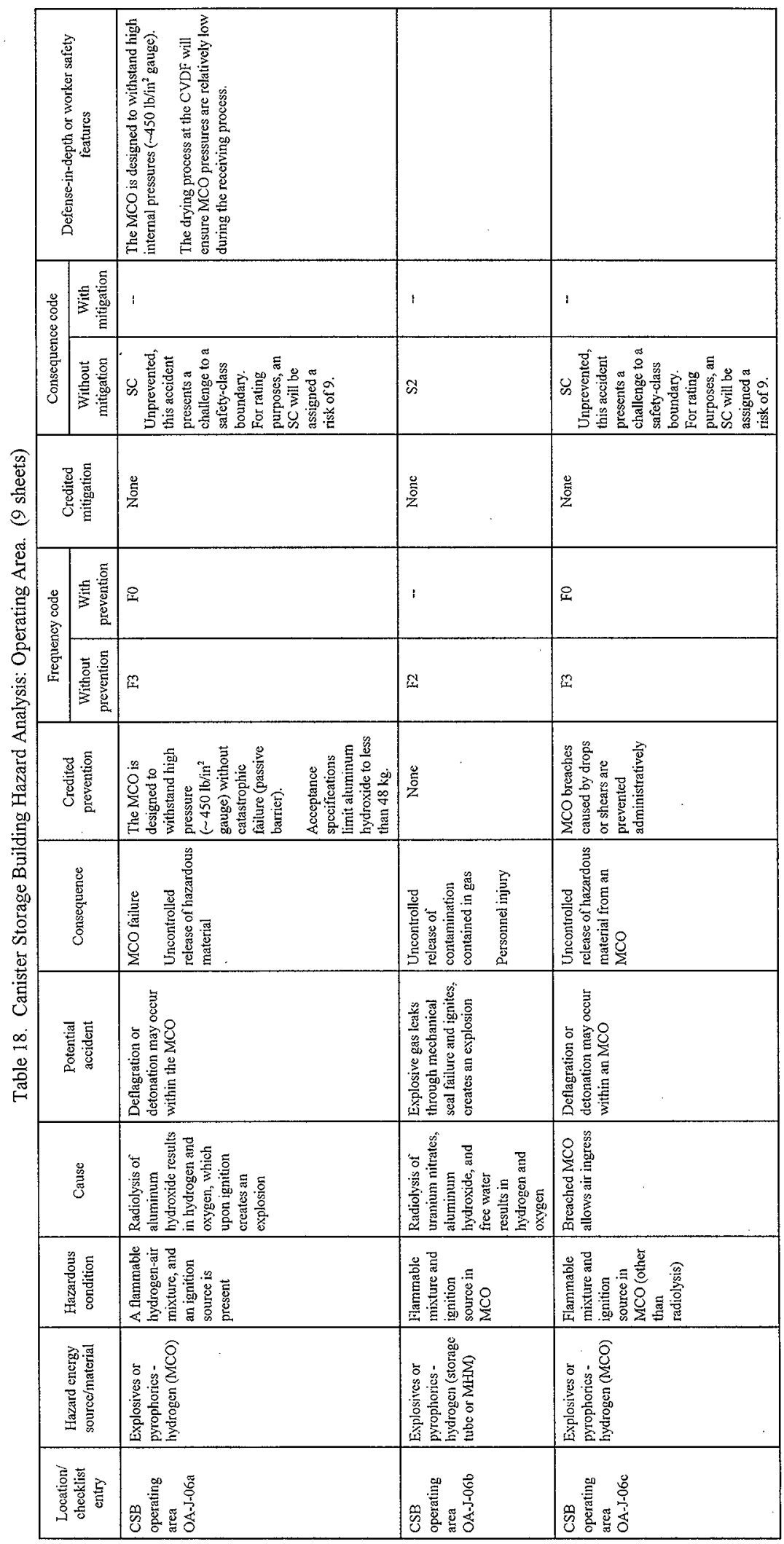




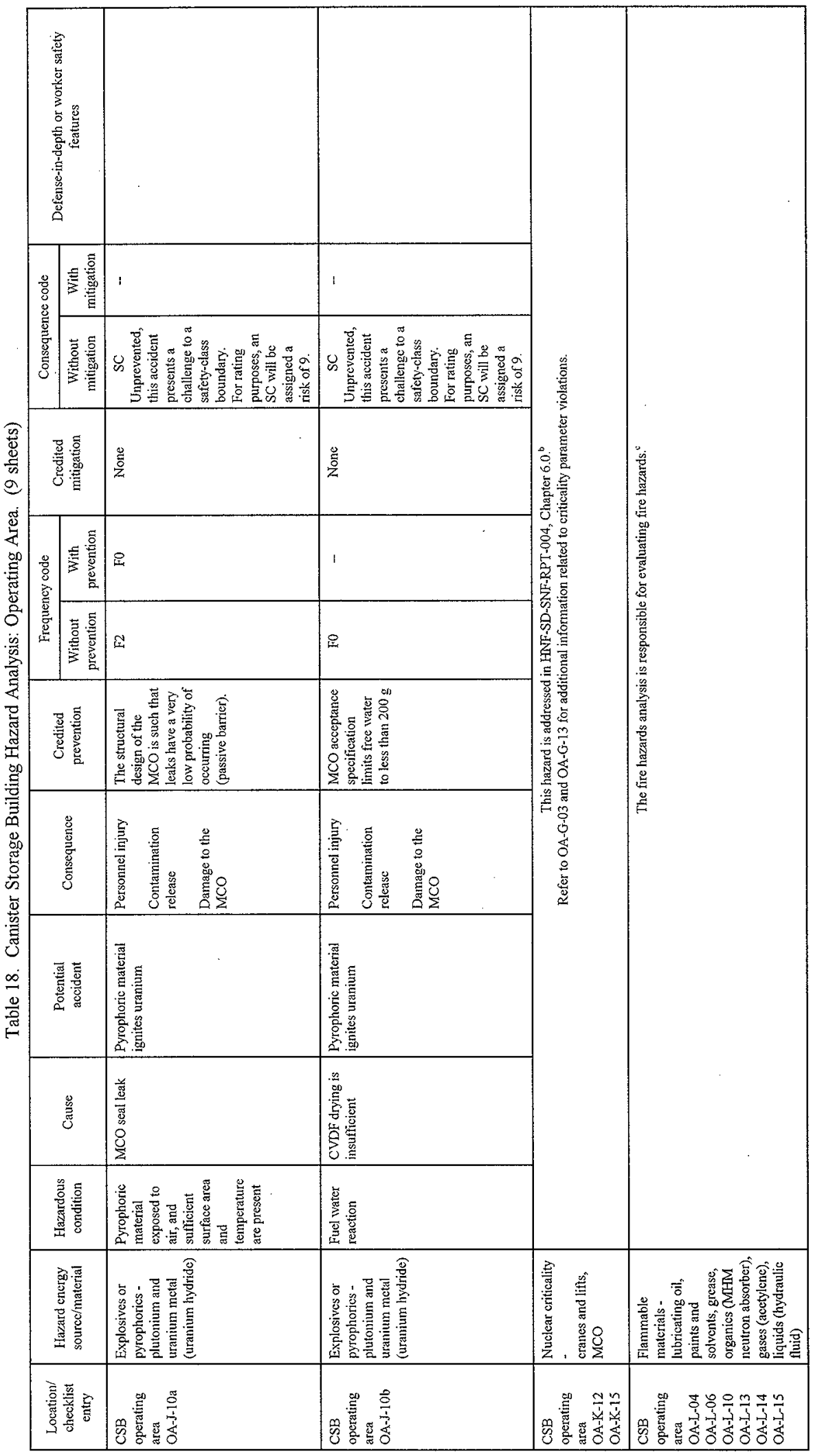


HNF-SD-SNF-HIE-001 REV 1

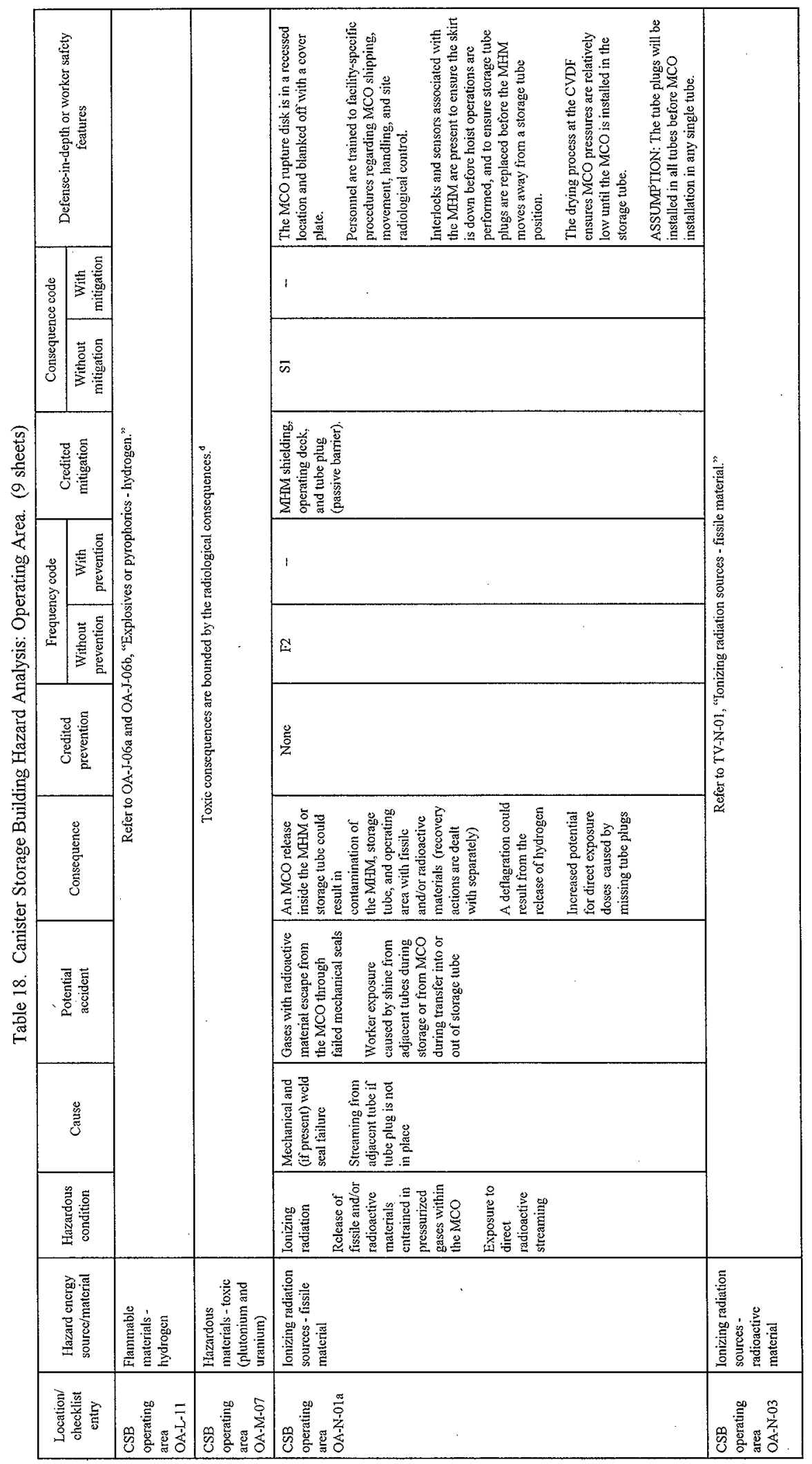




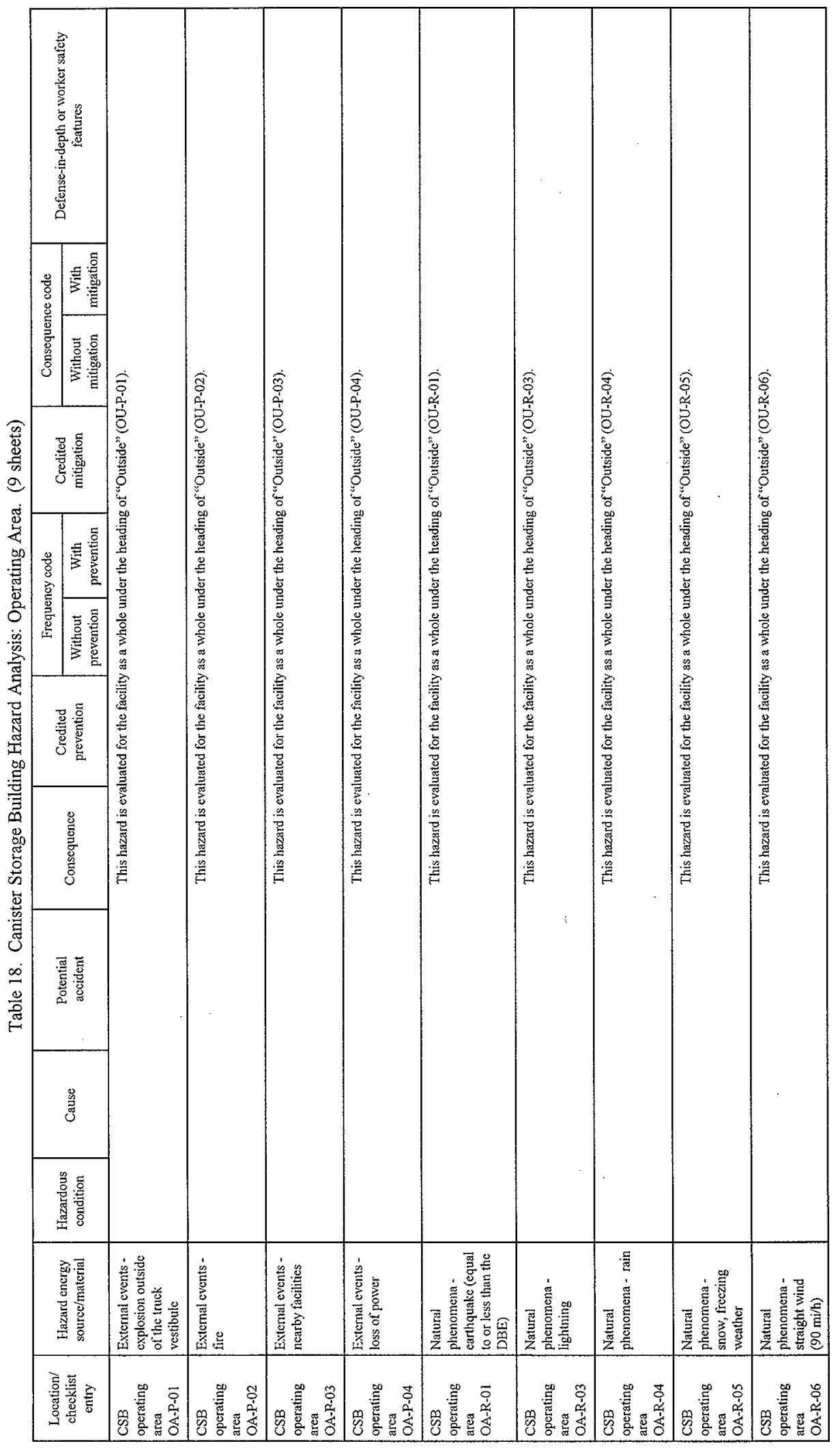




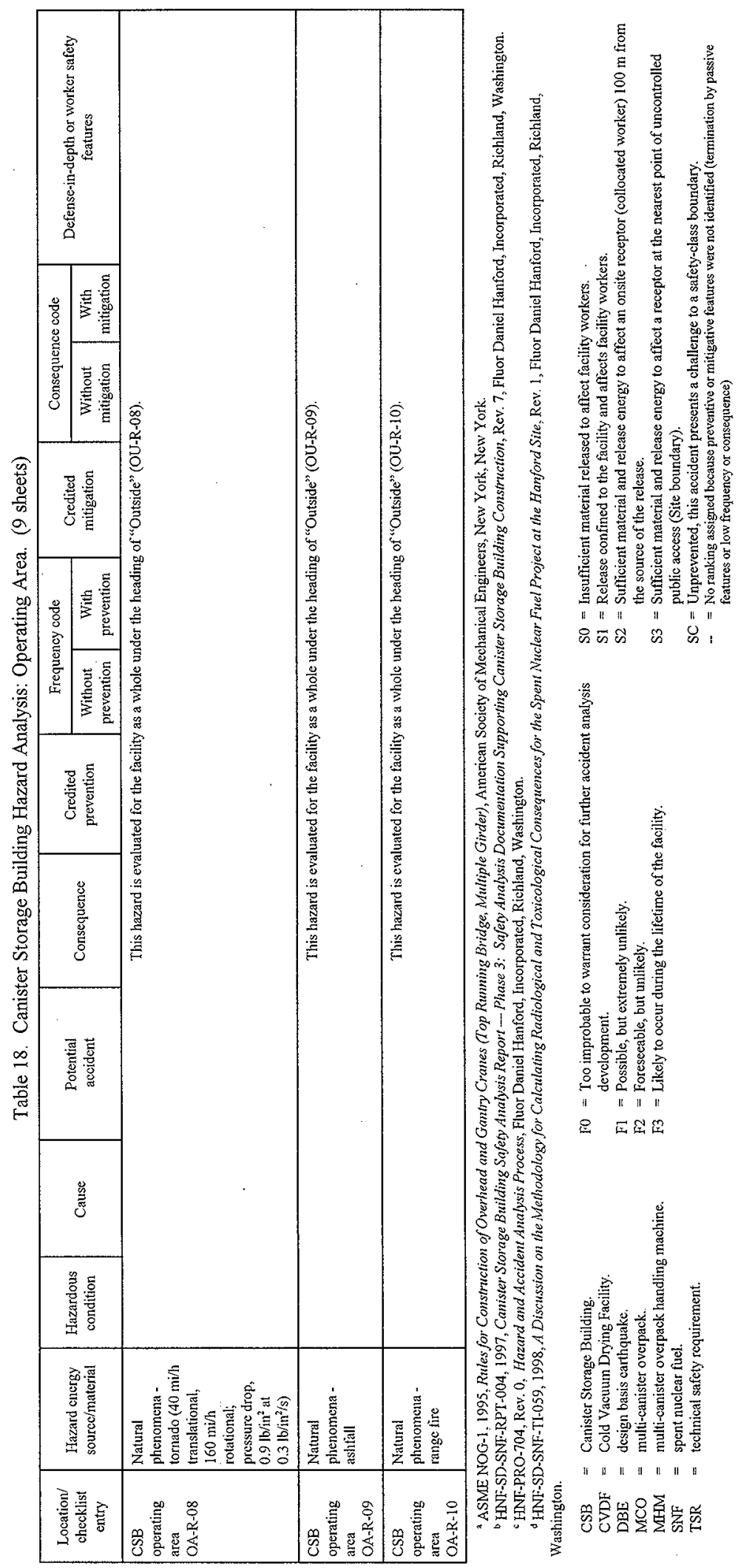


HNF-SD-SNF-HIE-001 REV I

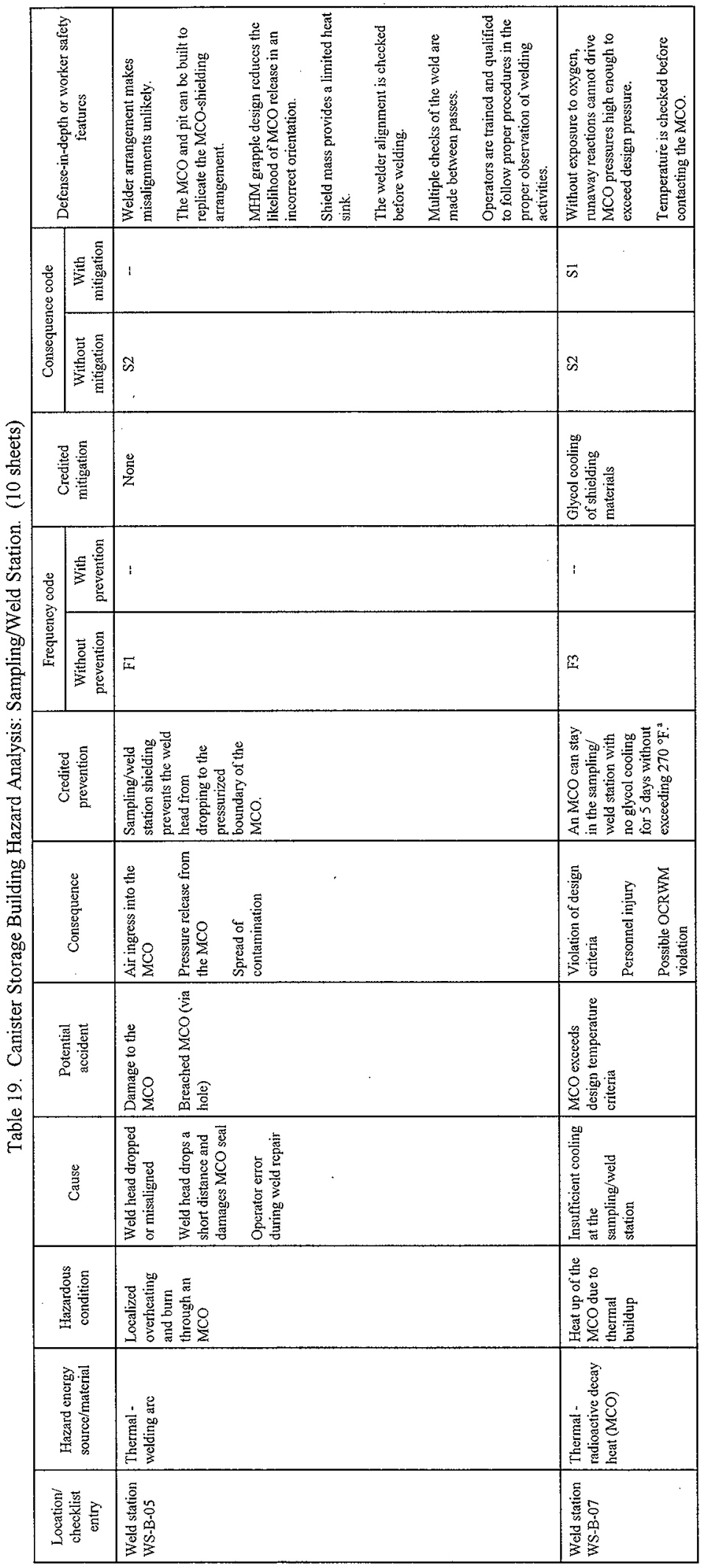


HNF-SD-SNF-HIE-001 REV 1

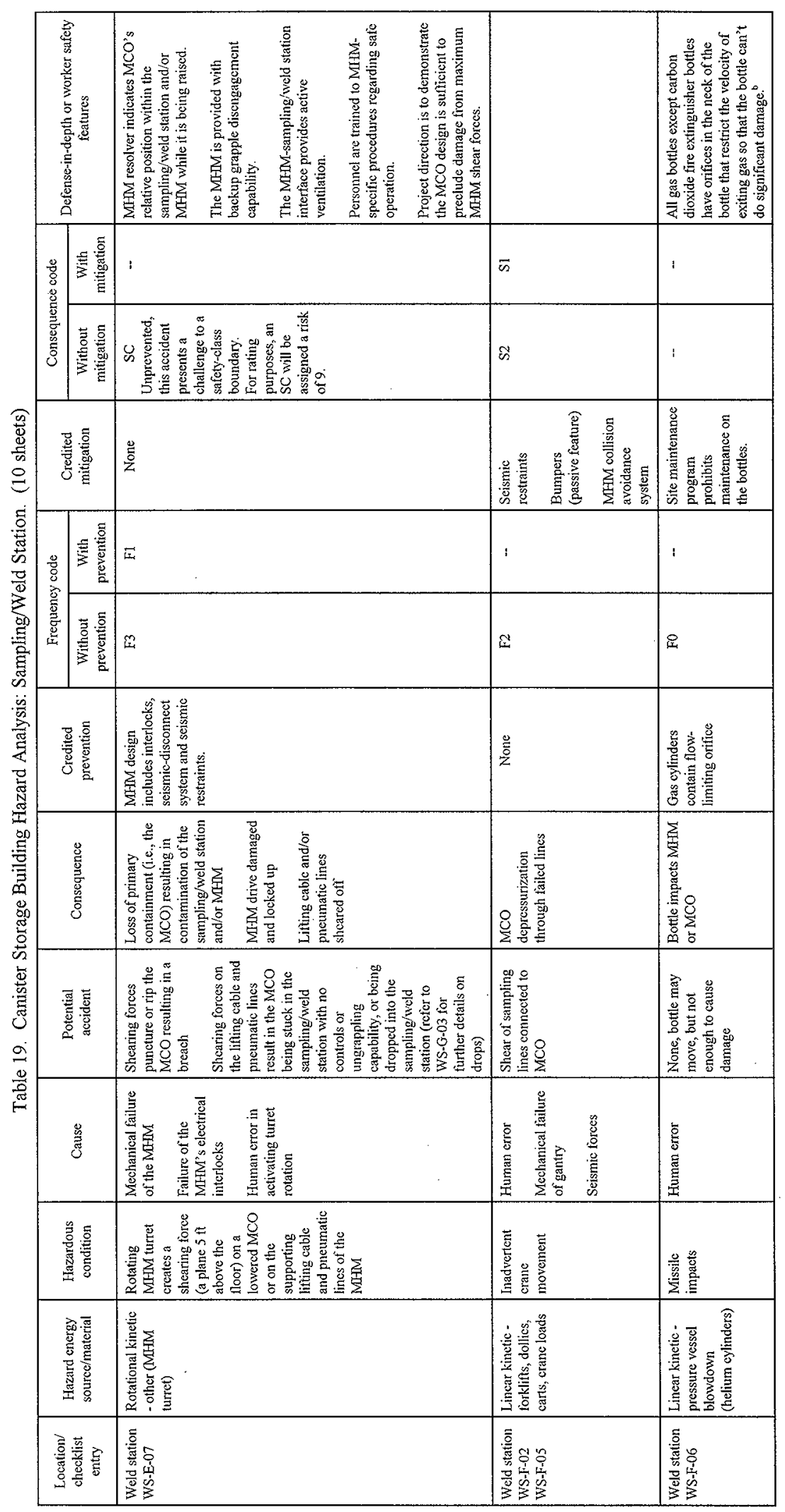


HNF-SD-SNF-HIE-001 REV 1

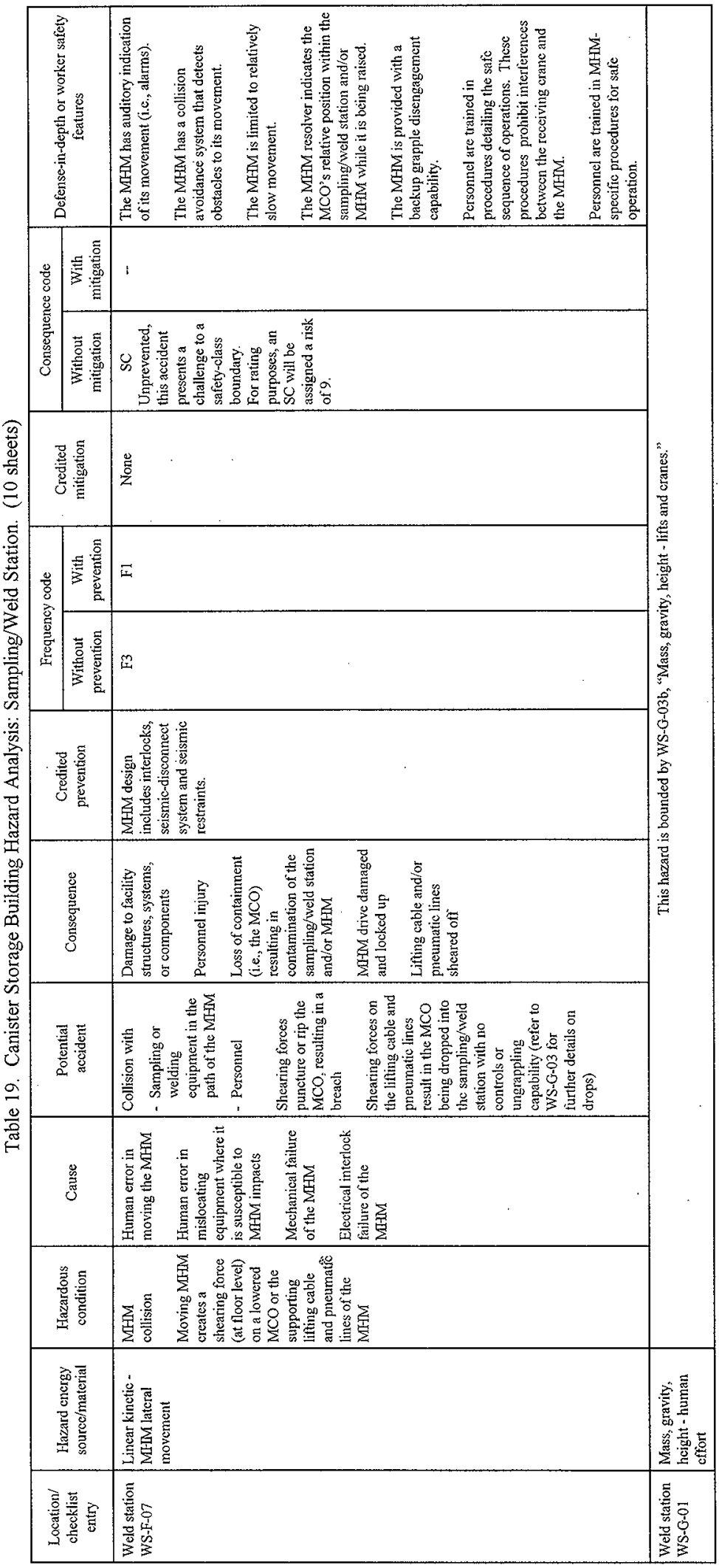


HNF-SD-SNF-HIE-001 REV 1

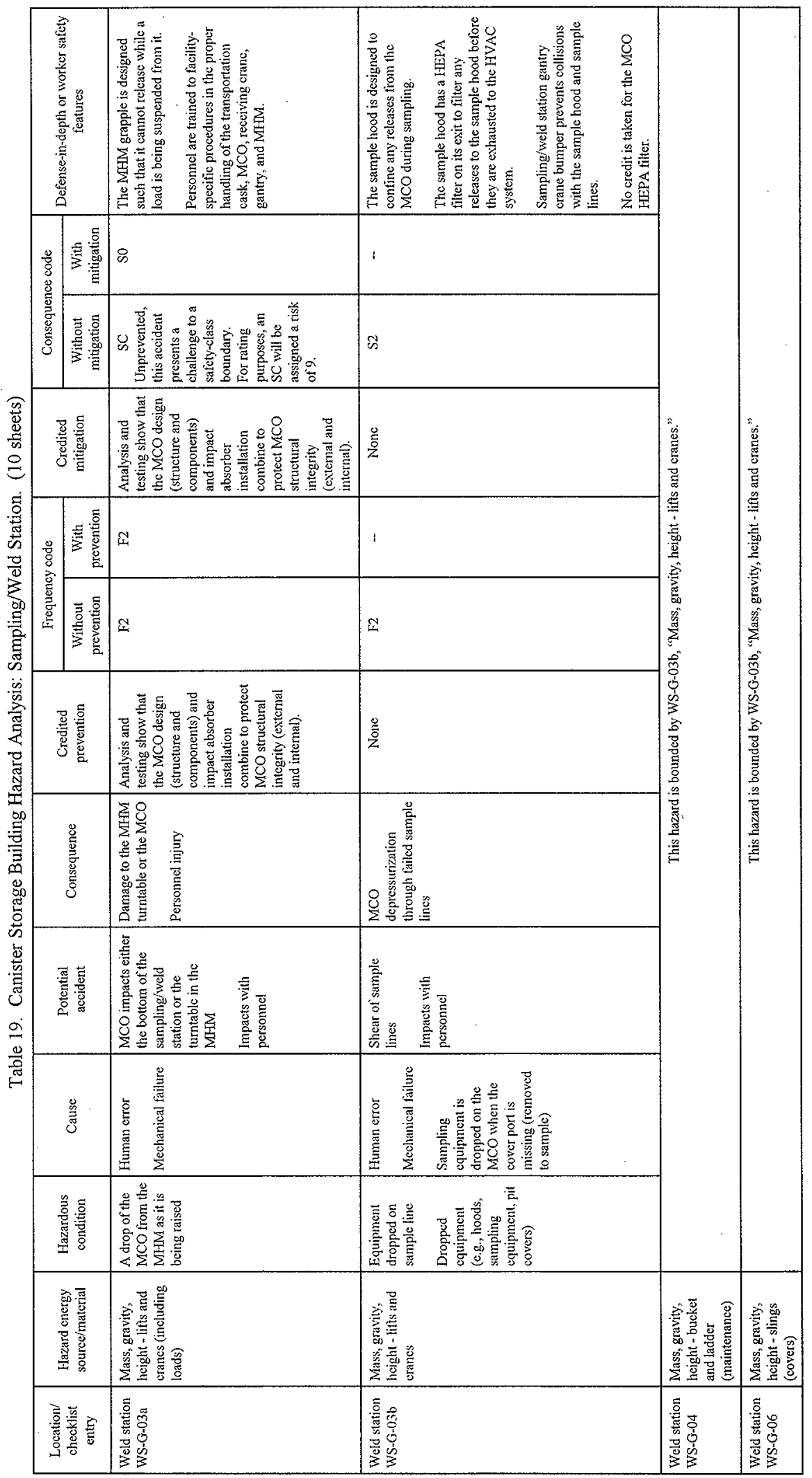


HNF-SD-SNF-HIE-001 REV 1

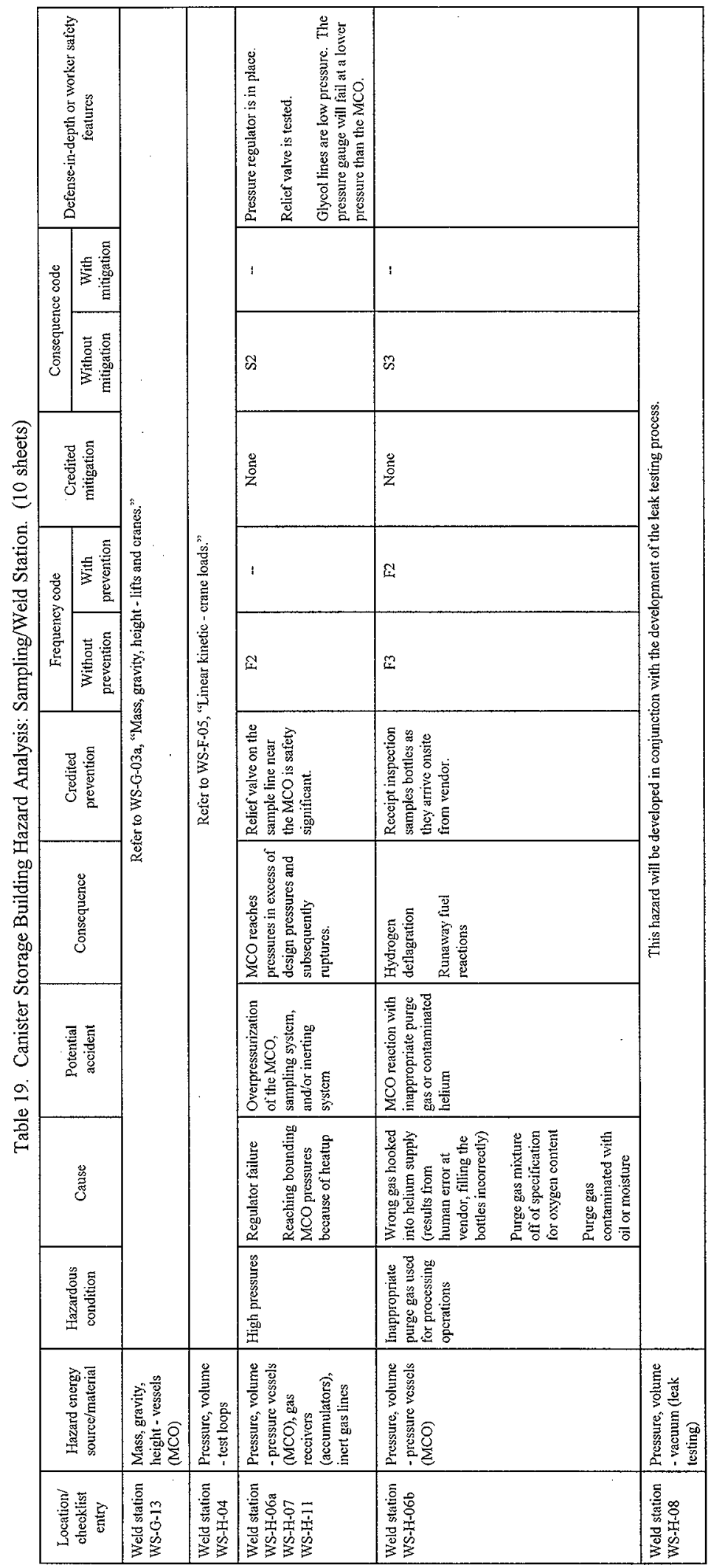


HNF-SD-SNF-HIE-001 REV 1

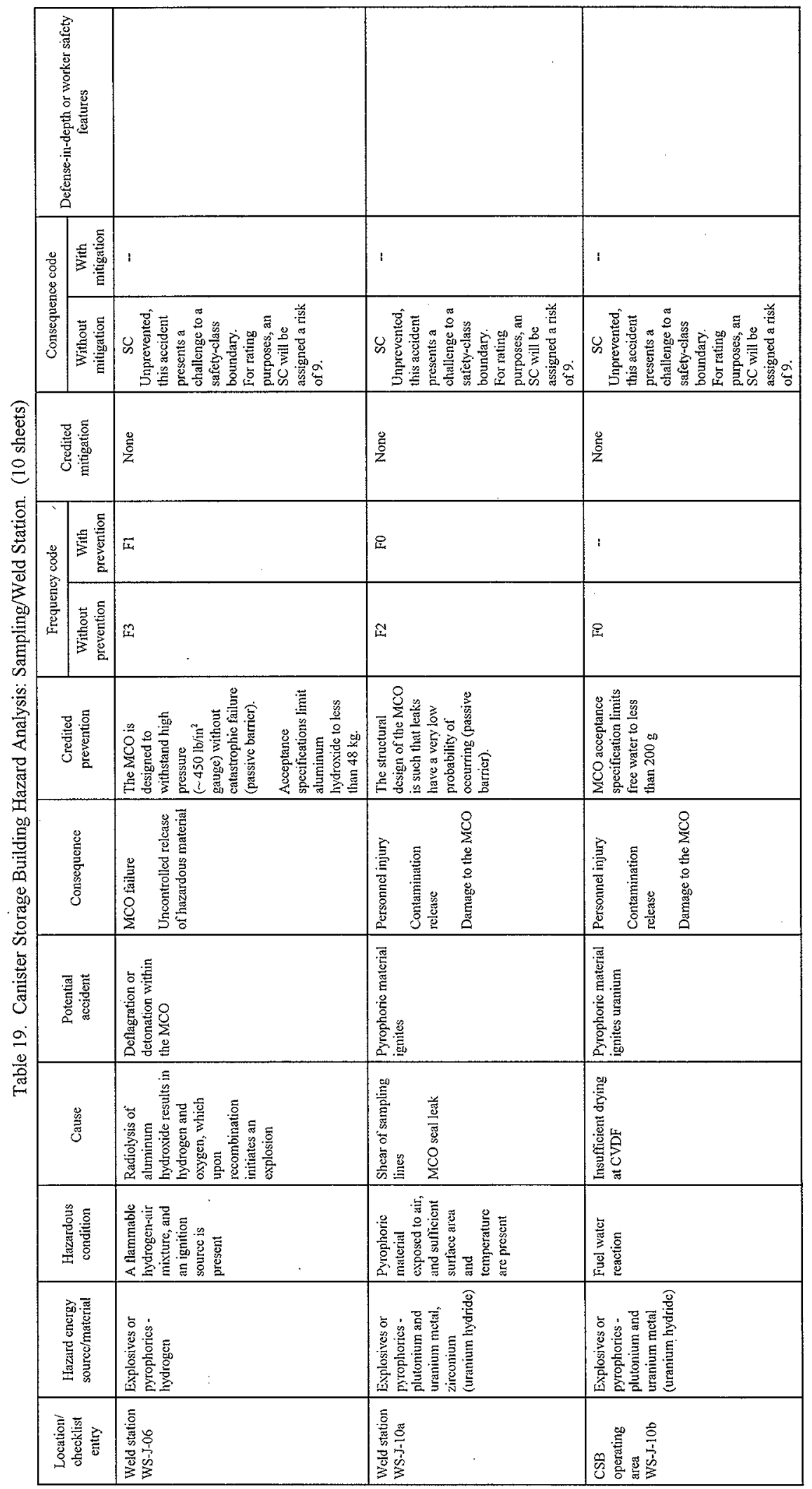


HNF-SD-SNF-HIE-001 REV 1

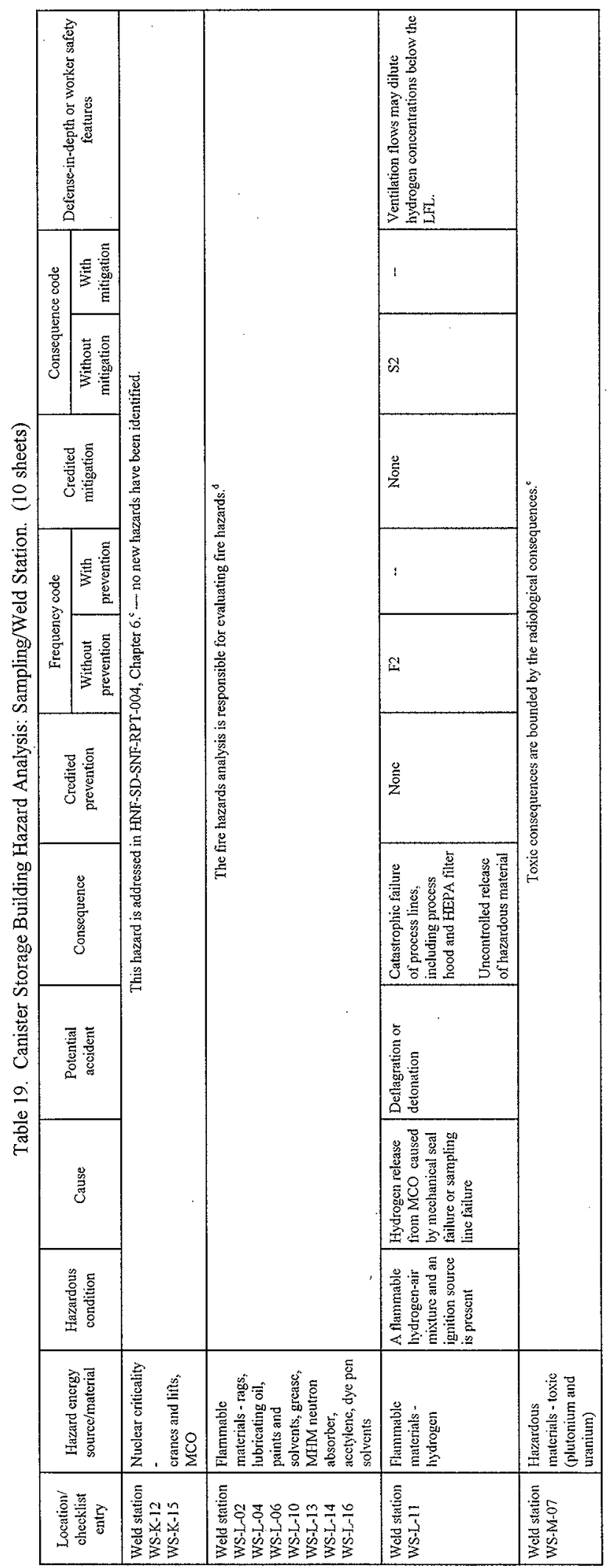


HNF-SD-SNF-HIE-001 REV I

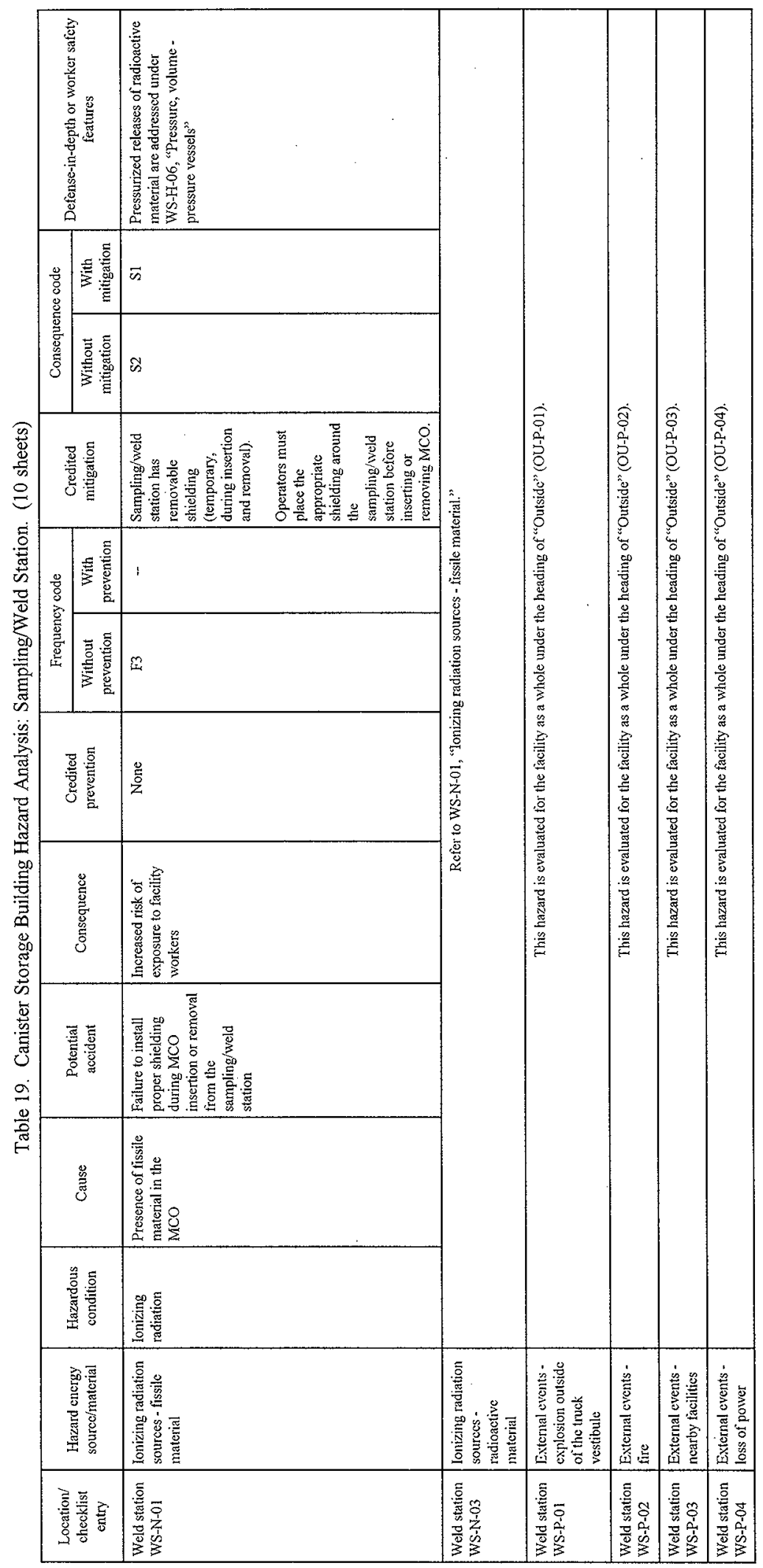




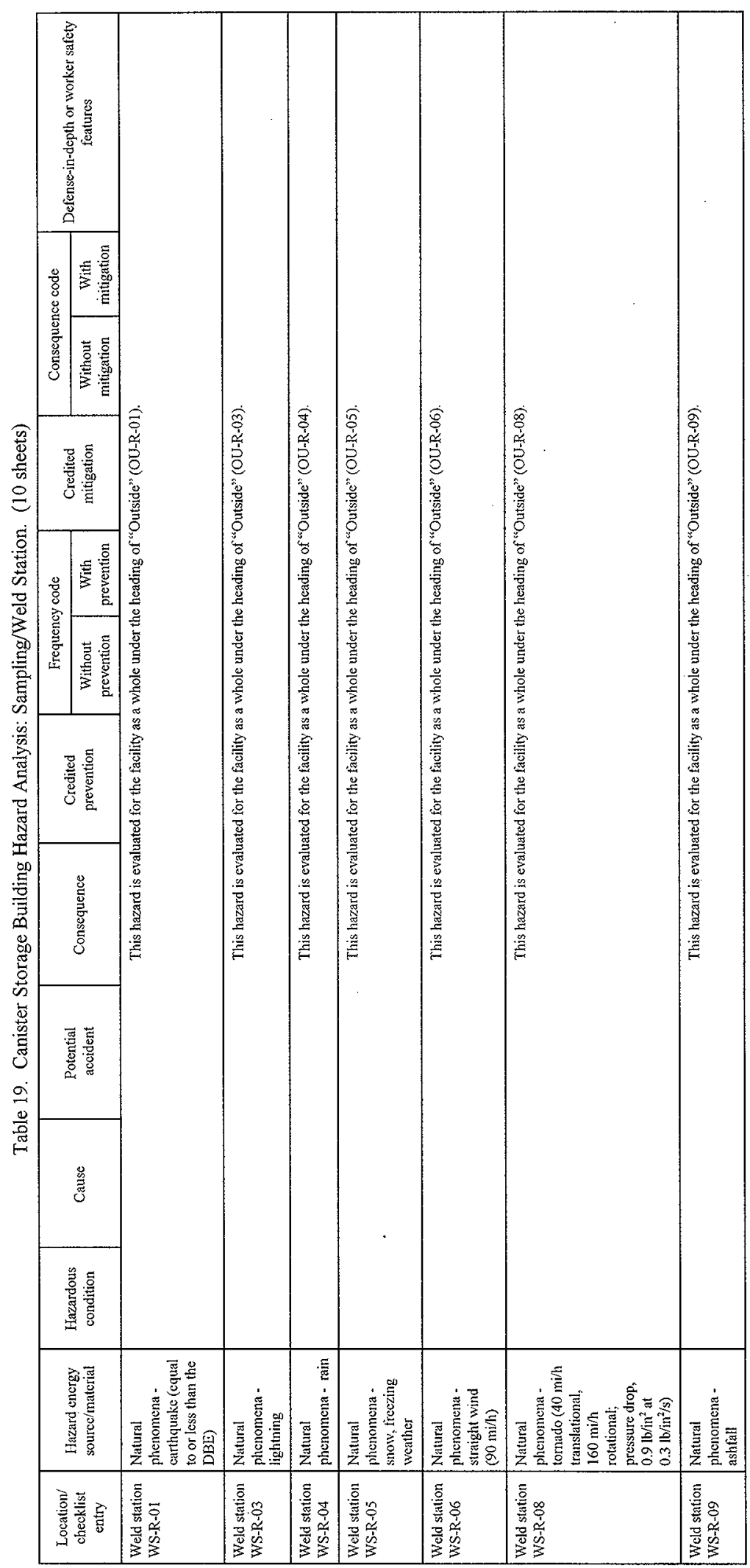




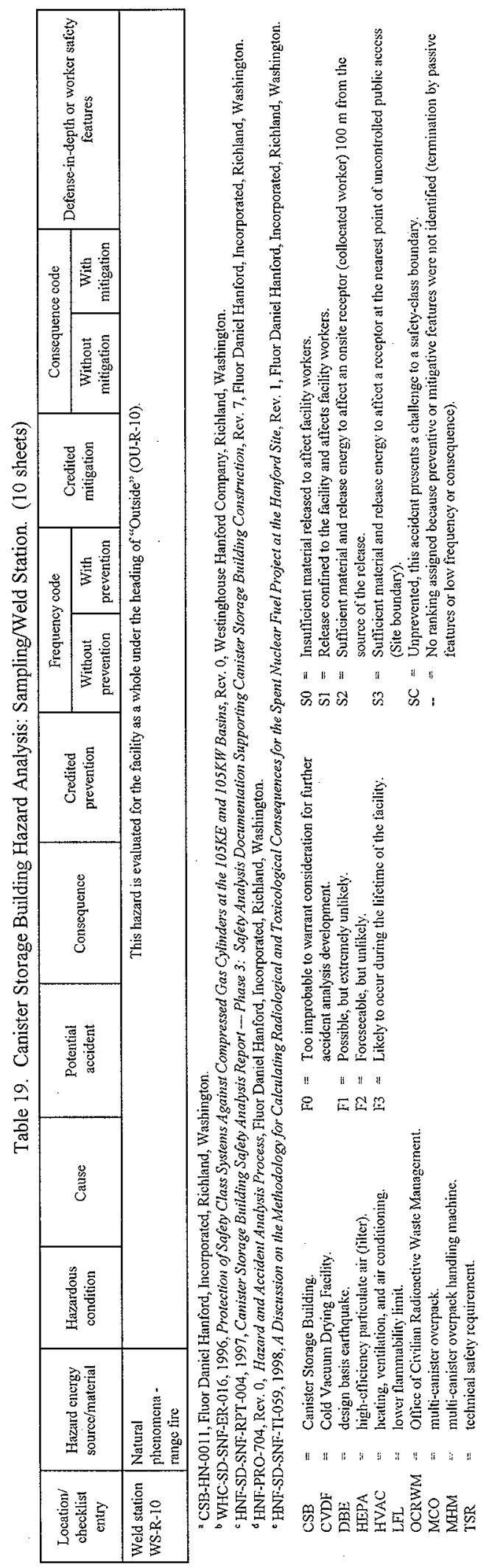




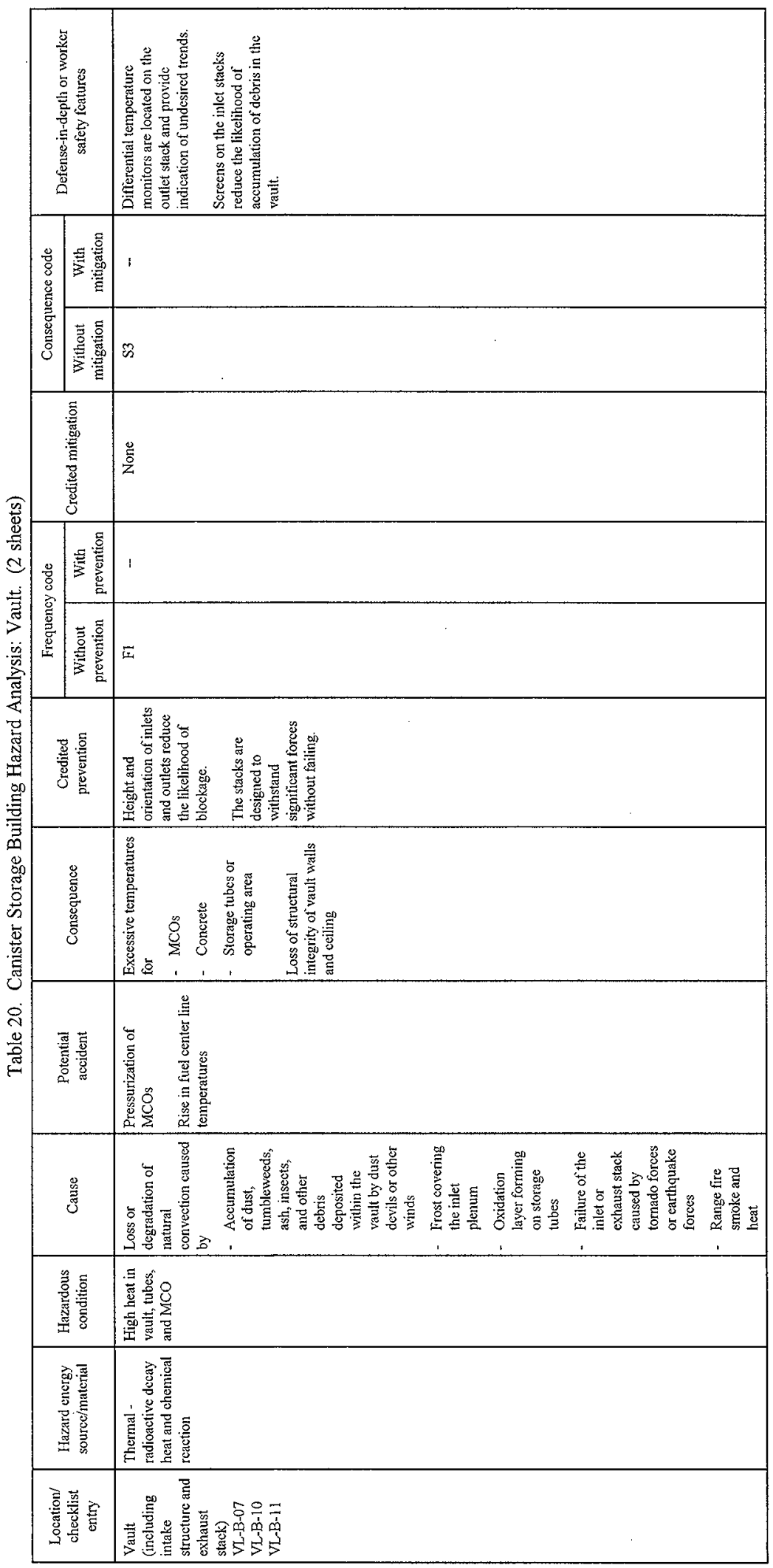




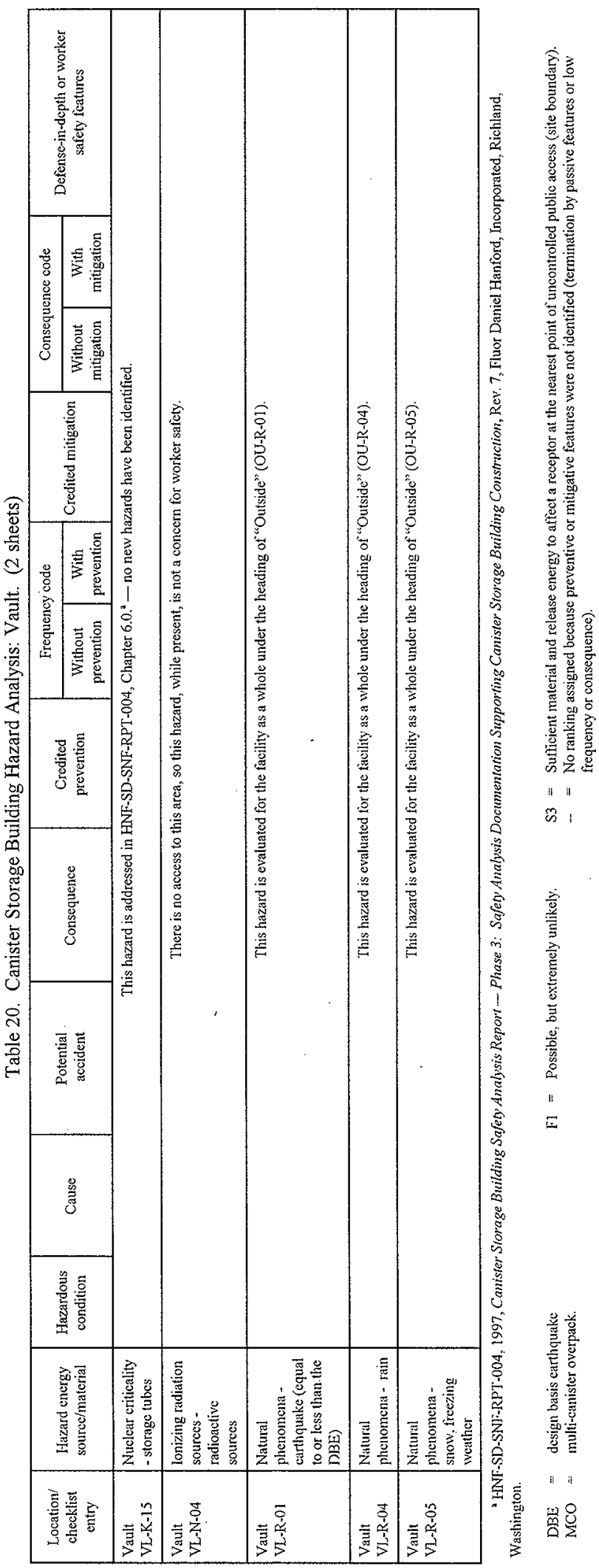




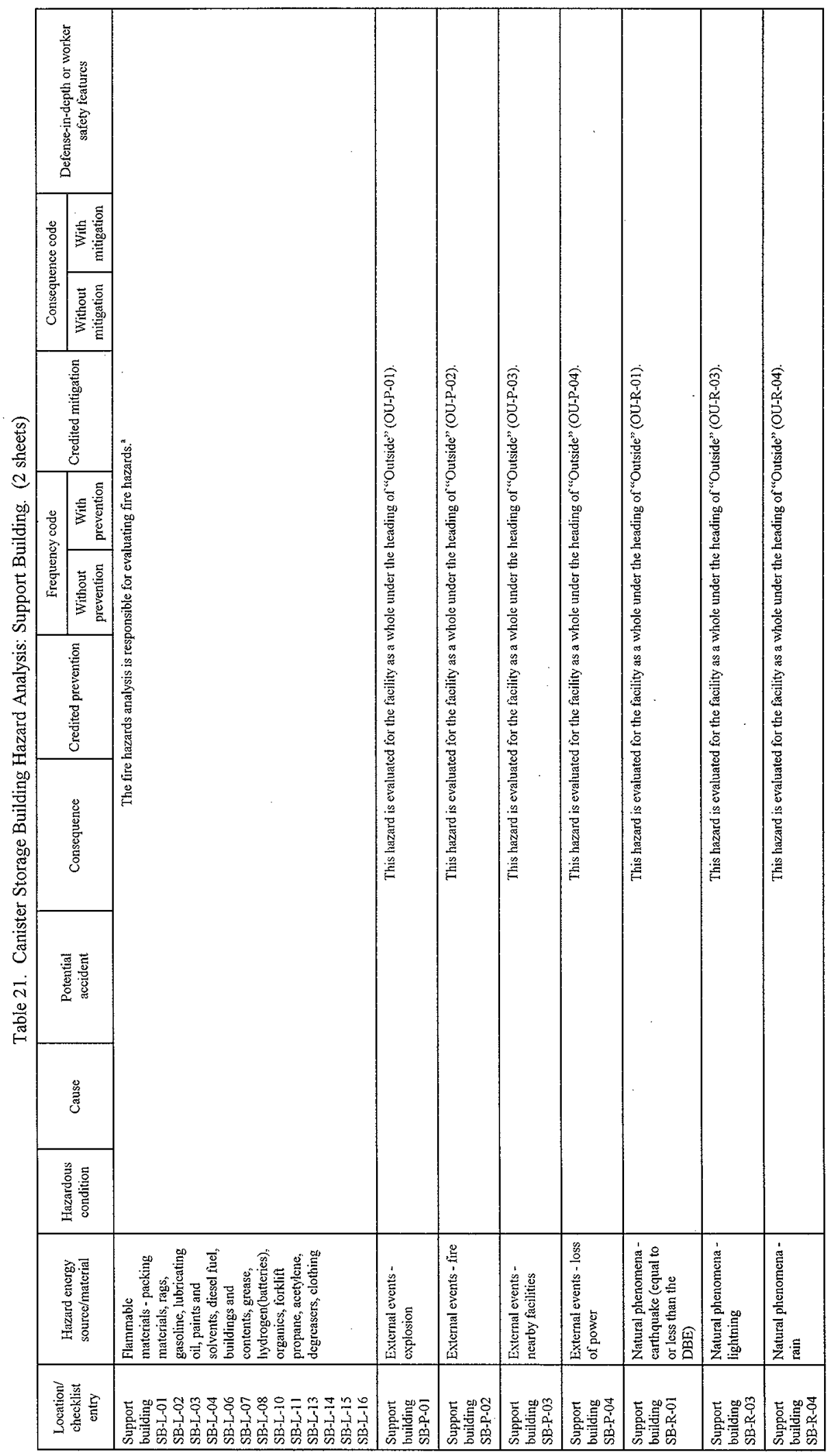


HNF-SD-SNF-HIE-001 REV 1

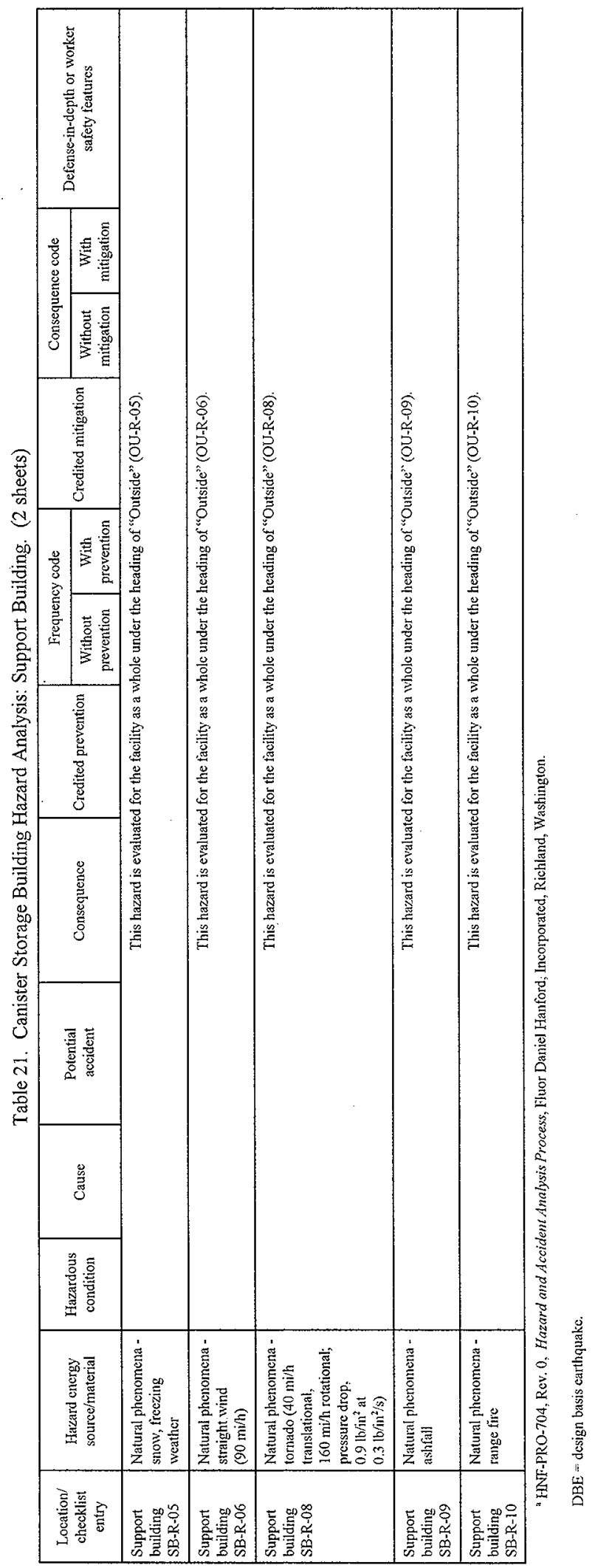


HNF-SD-SNF-HIE-001 REV 1

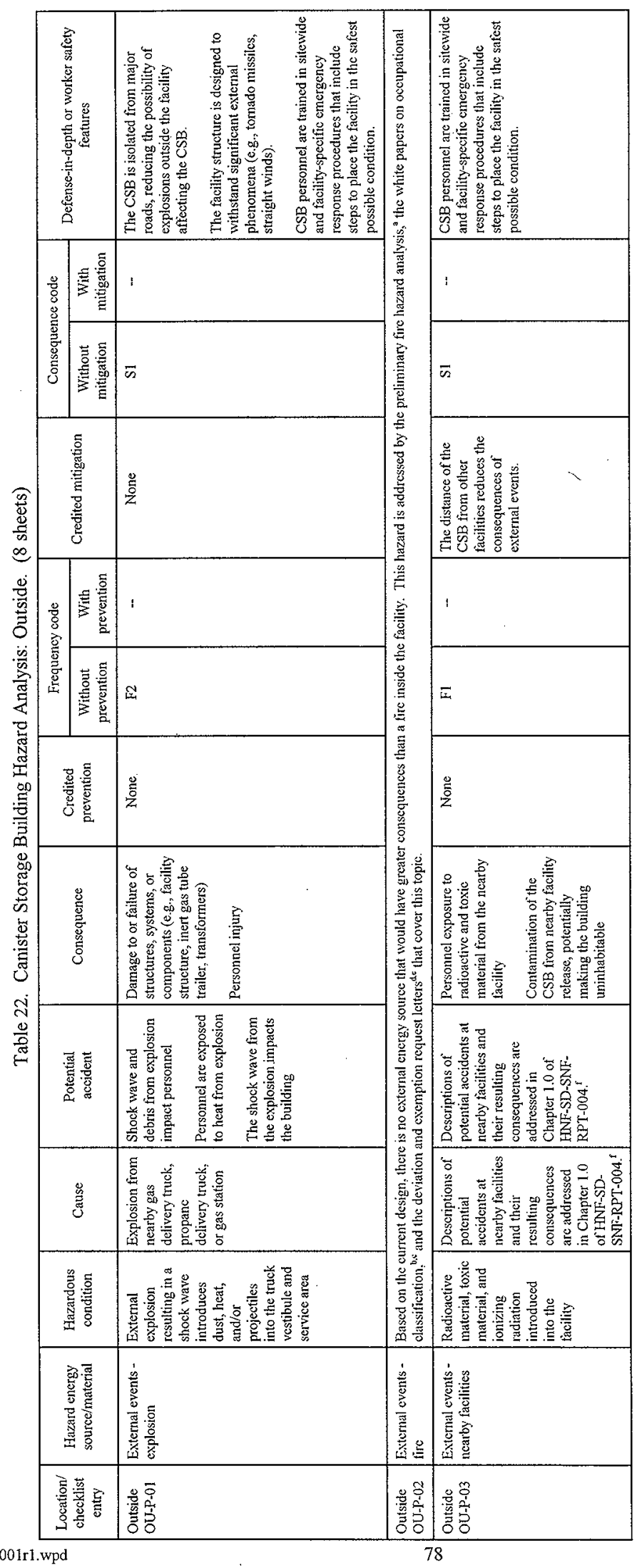


HNF-SD-SNF-HIE-001 REV 1

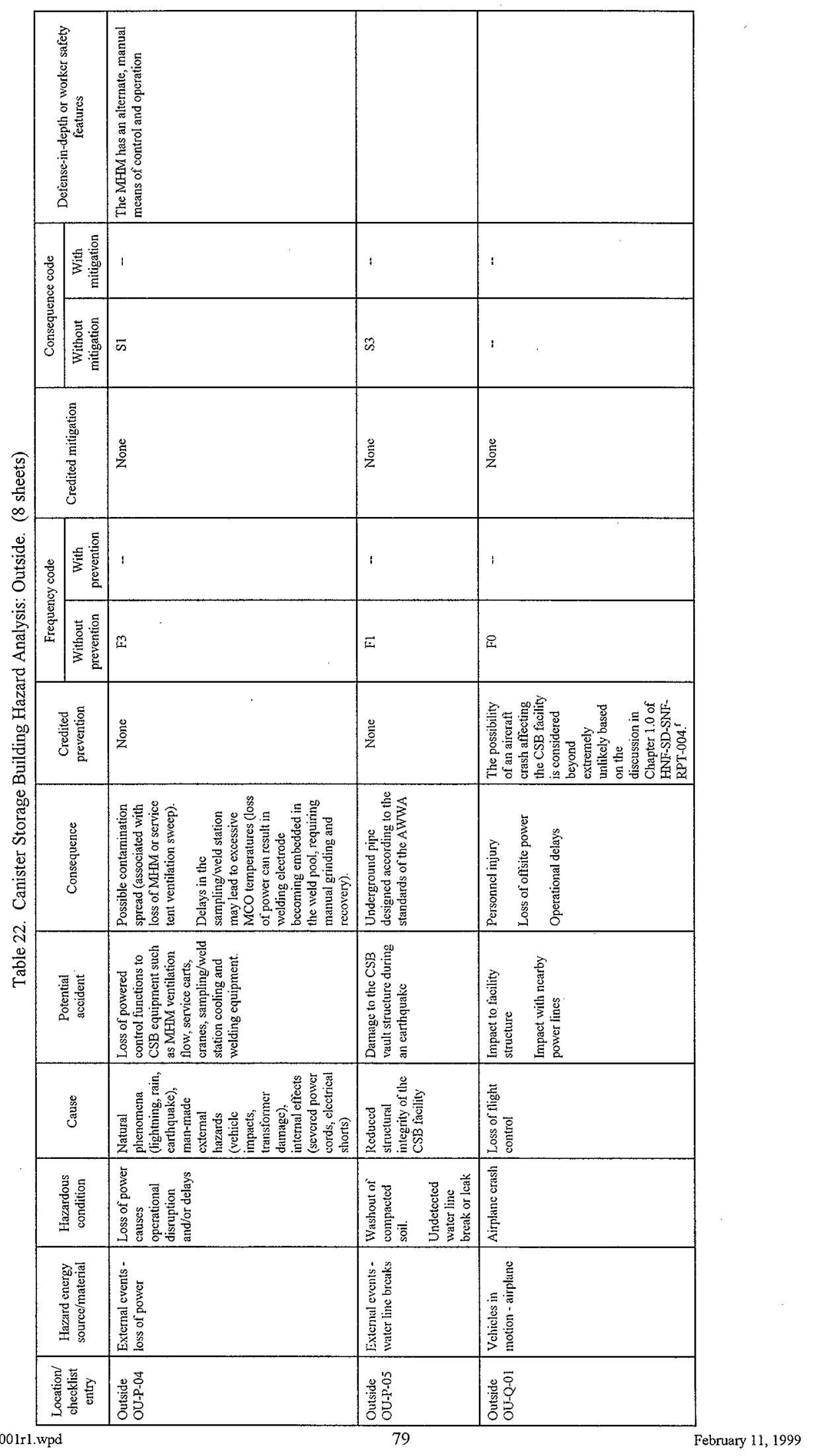


HNF-SD-SNF-HIE-001 REV 1

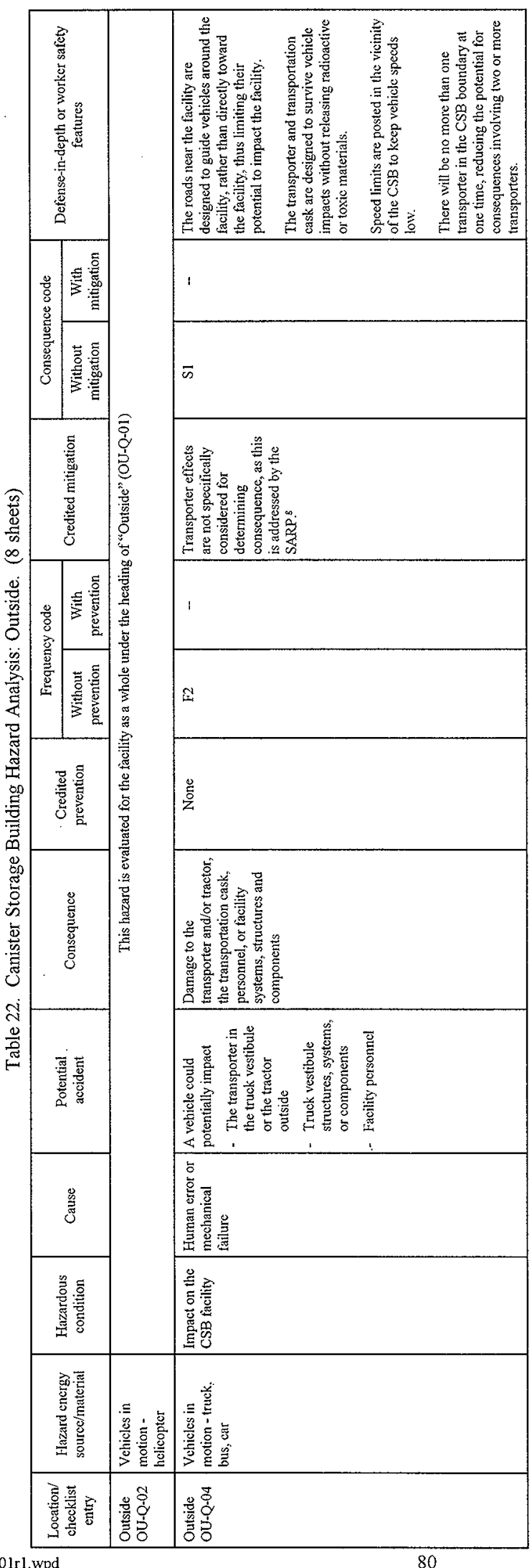


HNF-SD-SNF-HIE-001 REV 1

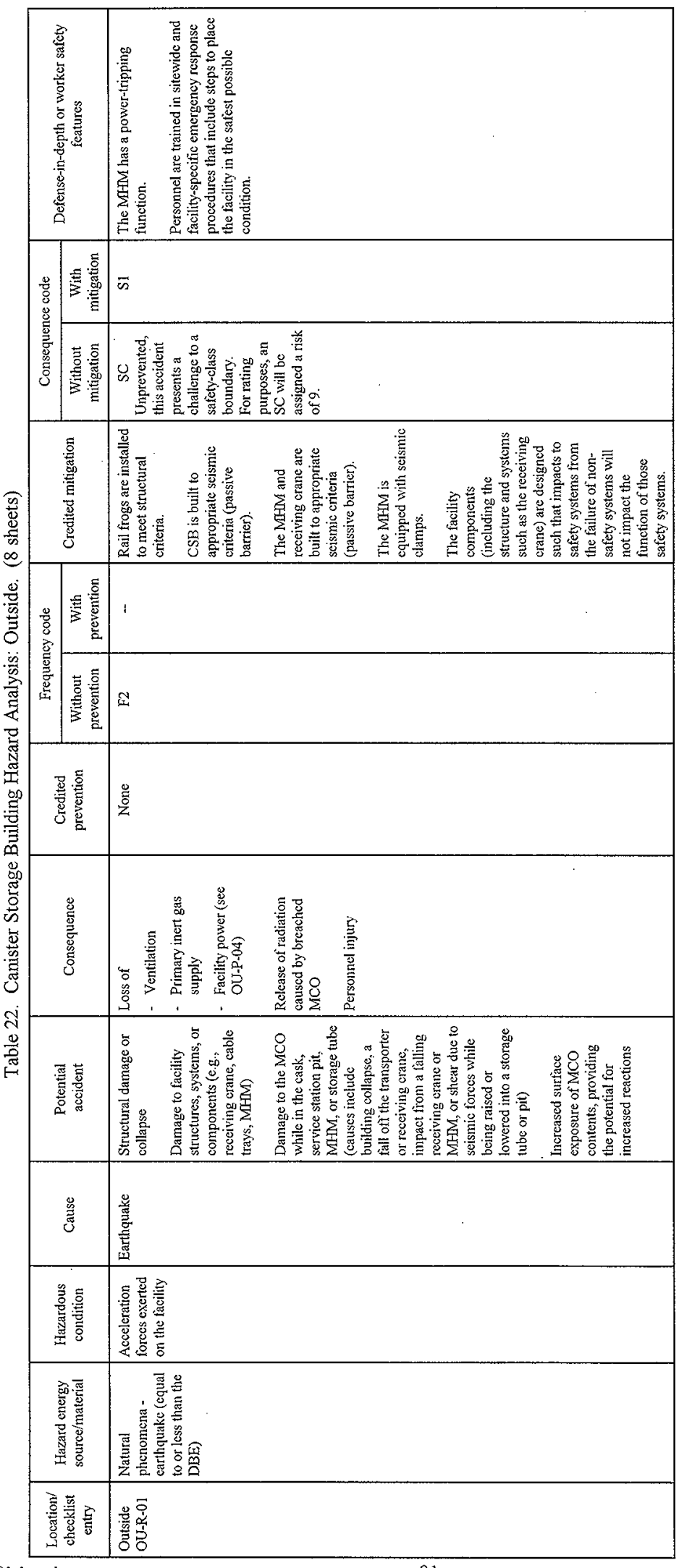


HNF-SD-SNF-HIE-001 REV 1

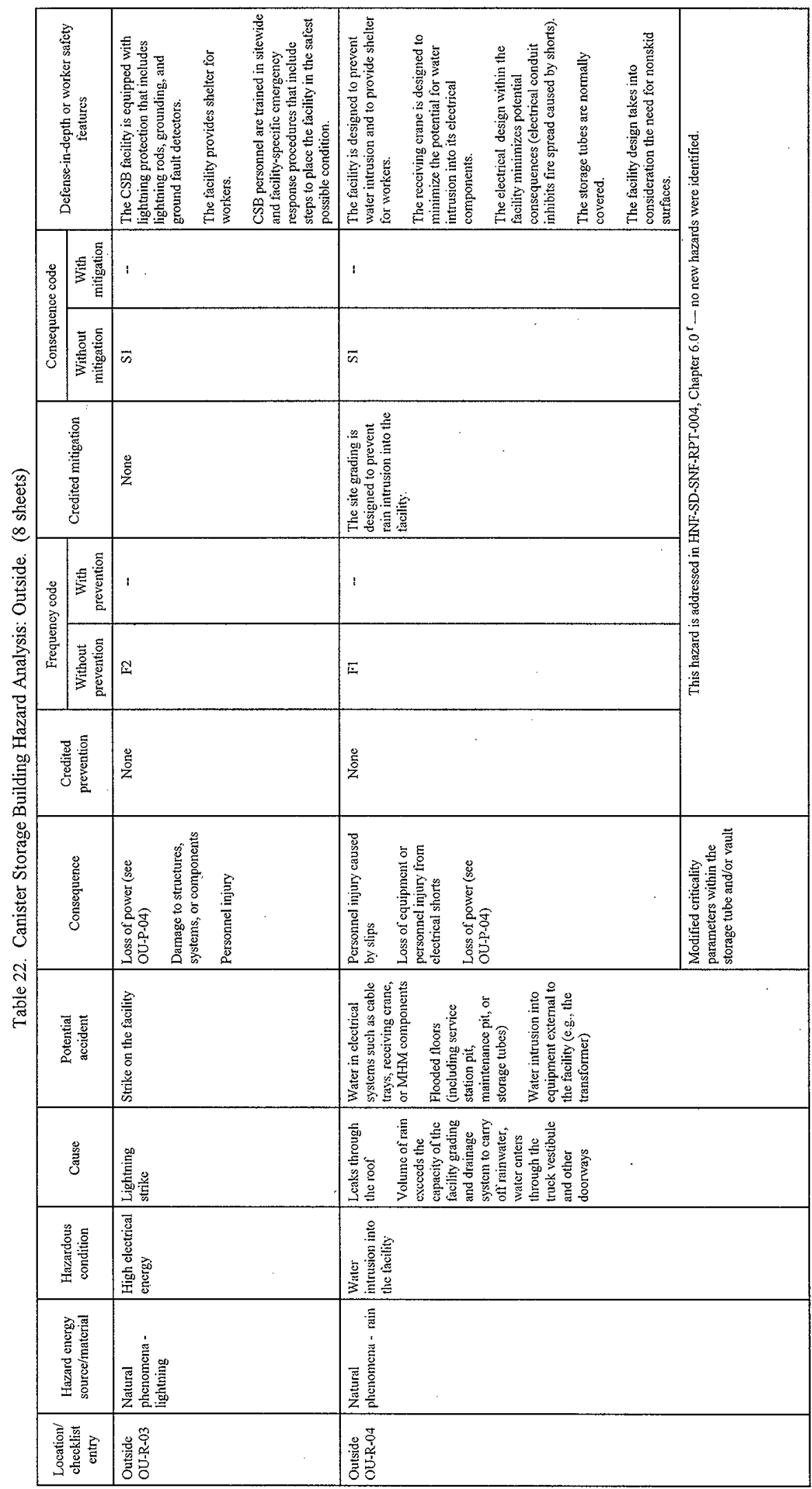


HNF-SD-SNF-HIE-001 REV I

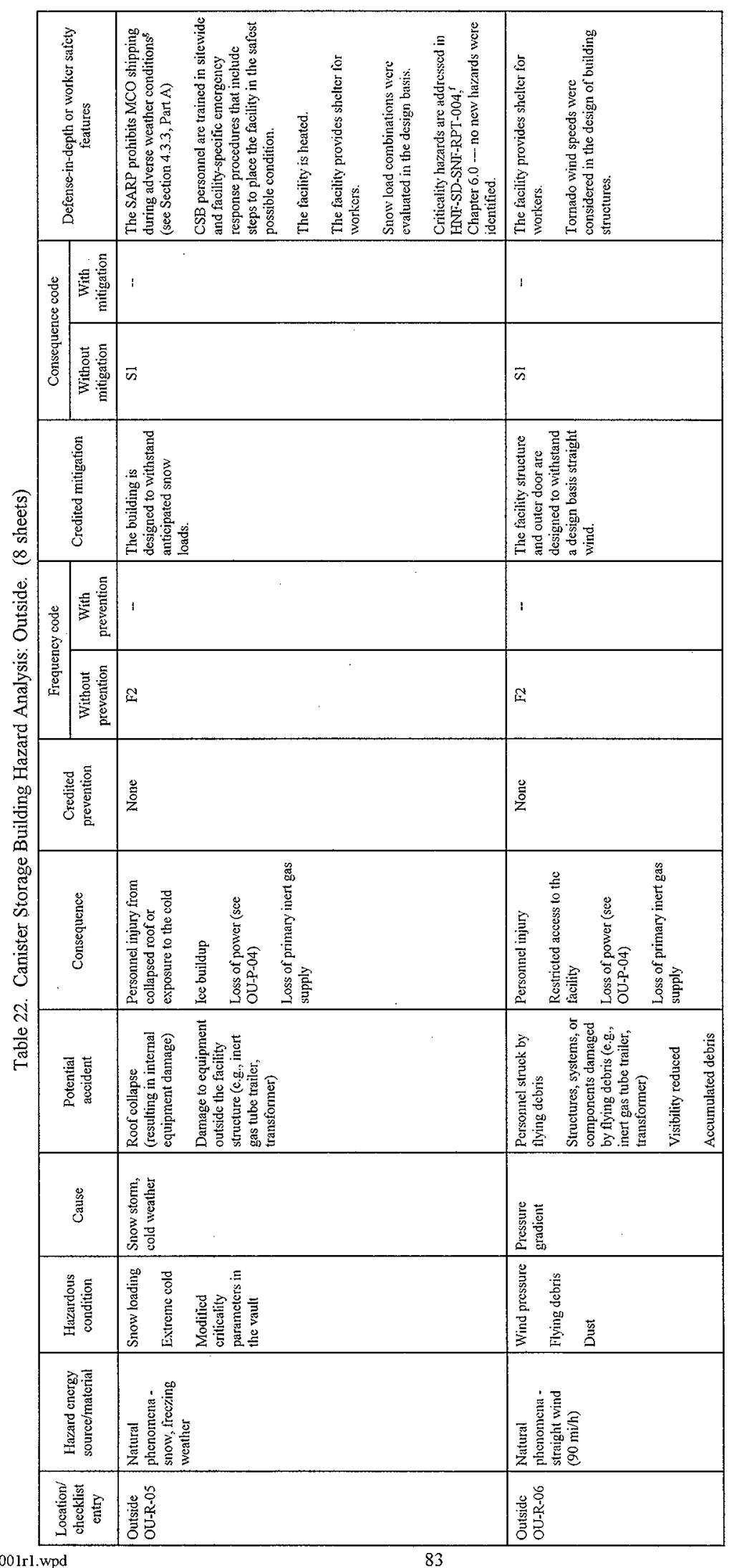


HNF-SD-SNF-HIE-001 REV 1

\begin{tabular}{|c|c|c|c|}
\hline 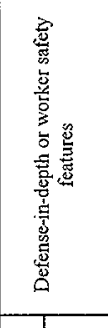 & 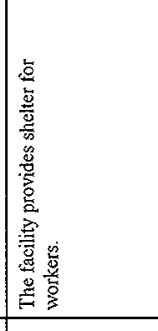 & 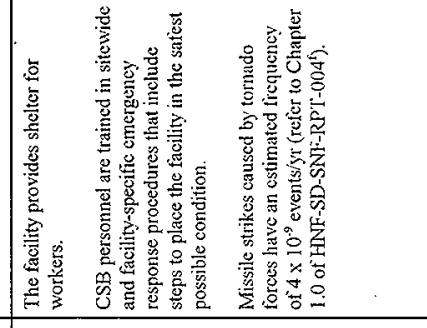 & 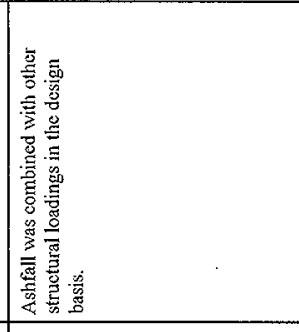 \\
\hline 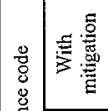 & $:$ & : & : \\
\hline 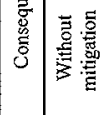 & कs & $\bar{s}$ & $\bar{\omega}$ \\
\hline 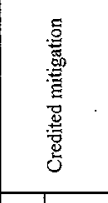 & \begin{tabular}{|l}
\multirow{2}{*}{} \\
产
\end{tabular} & 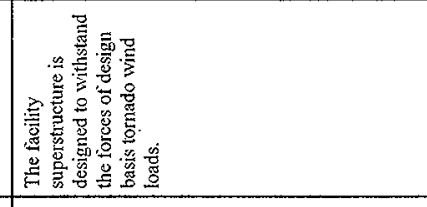 & 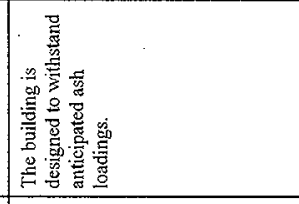 \\
\hline 章 & : & : & 1 \\
\hline 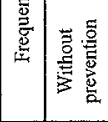 & $m$ & $\bar{E}$ & $\Phi$ \\
\hline 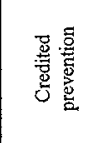 & 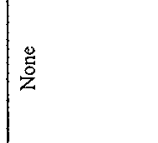 & \begin{tabular}{|l}
$\frac{\mathscr{0}}{\tilde{z}}$ \\
\end{tabular} & $\begin{array}{l}\stackrel{\circ}{\circ} \\
\bar{\delta}\end{array}$ \\
\hline 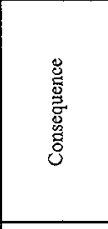 & 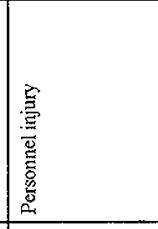 & 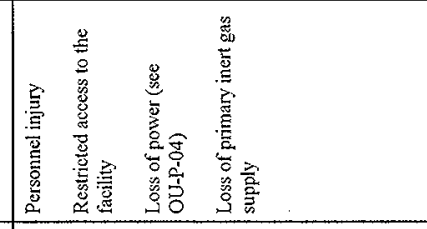 & 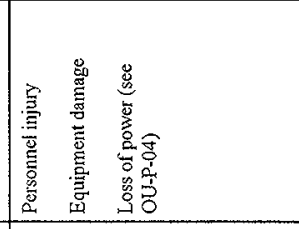 \\
\hline 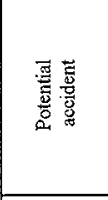 & 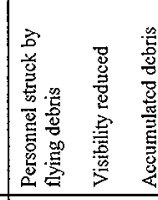 & 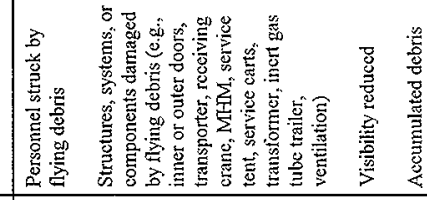 & 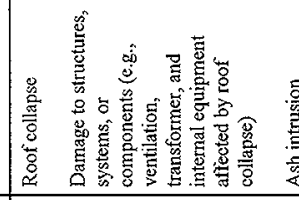 \\
\hline 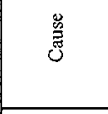 & 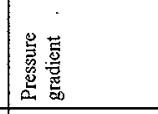 & 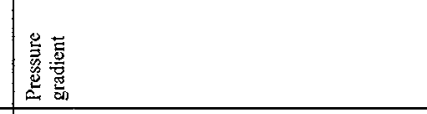 & 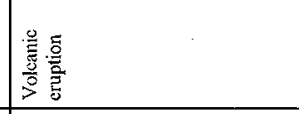 \\
\hline 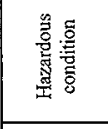 & 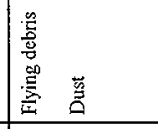 & 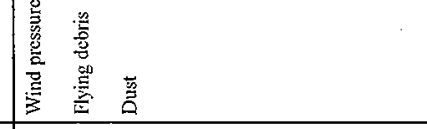 & 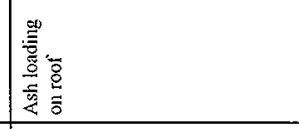 \\
\hline 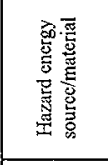 & 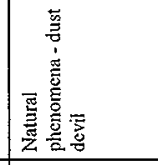 & 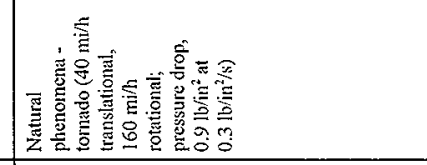 & 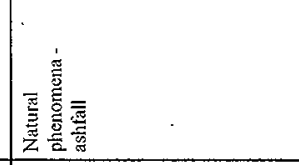 \\
\hline 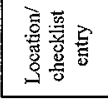 & 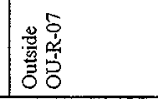 & 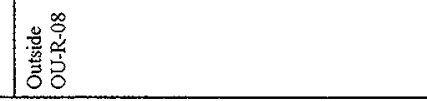 & 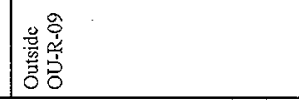 \\
\hline
\end{tabular}


HNF-SD-SNF-HIE-001 REV 1

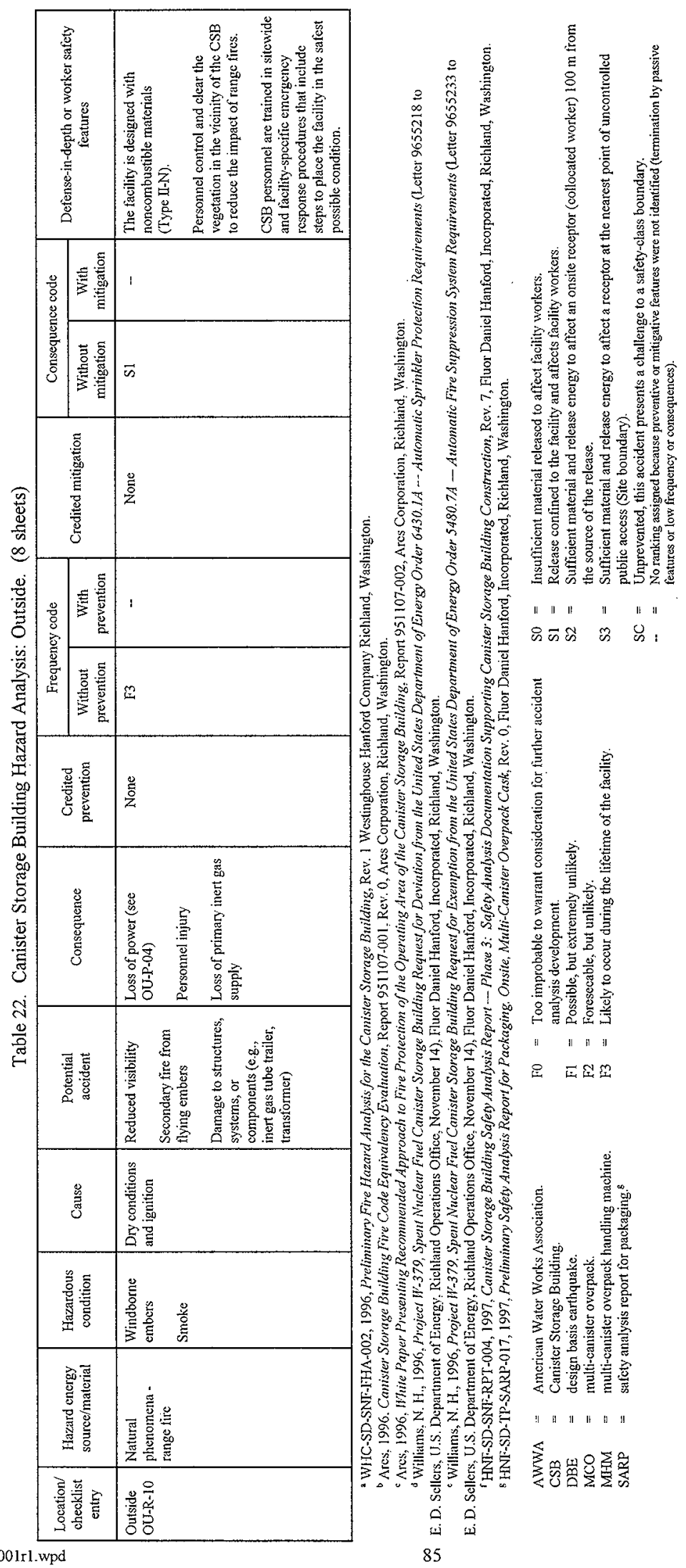


HNF-SD-SNF-HIE-001 REV 1

This page intentionally left blank. 
HNF-SD-SNF-HIE-001 REV 1

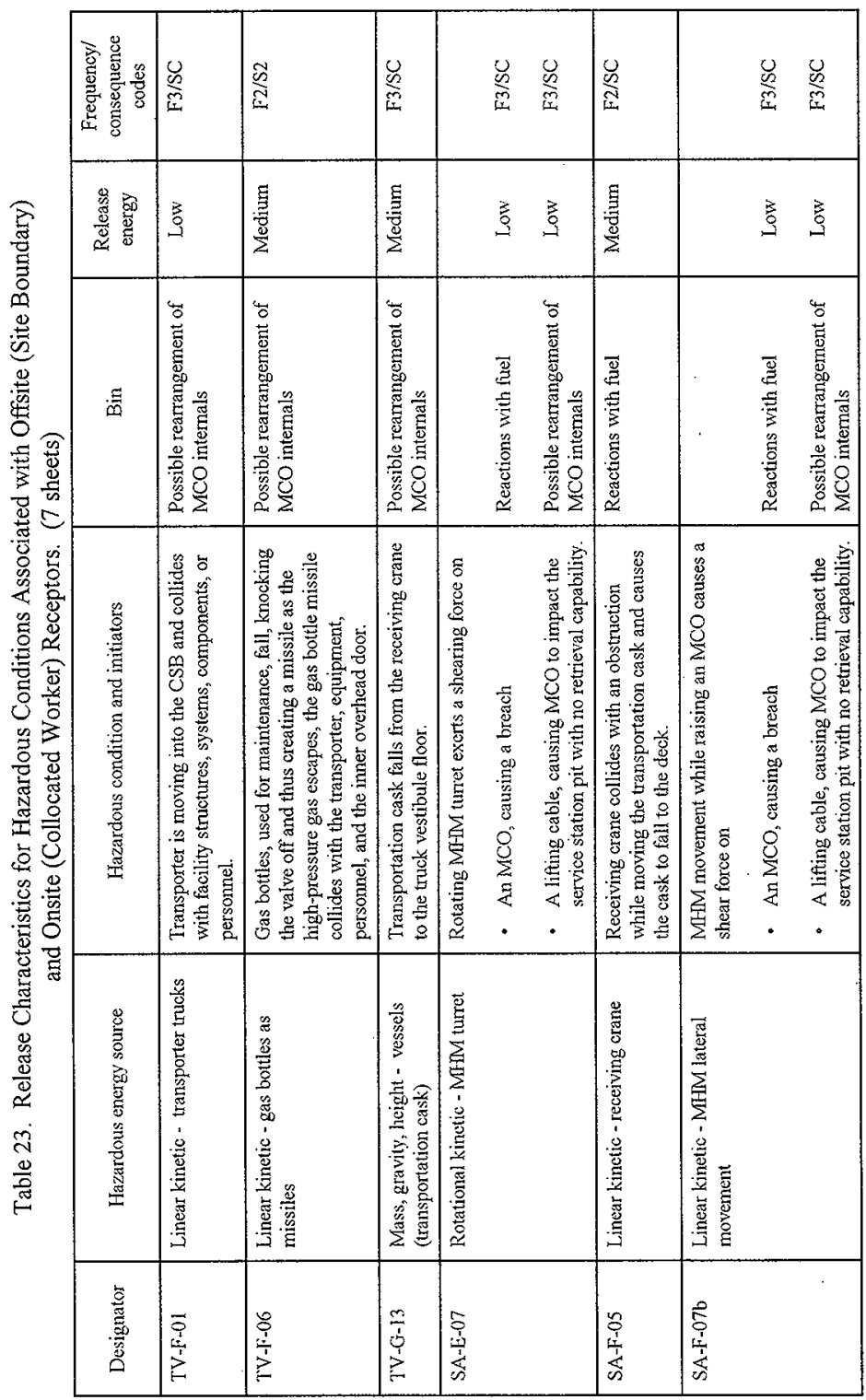


HNF-SD-SNF-HIE-001 REV 1

\begin{tabular}{|c|c|c|c|c|c|c|c|}
\hline 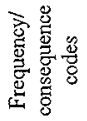 & & 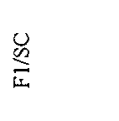 & $\underset{⿱ 丷}{\infty}$ & 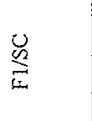 & & $\underset{0}{0}$ & 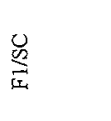 \\
\hline 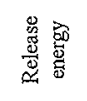 & & 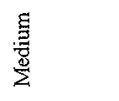 & 恶 & 萑 & & 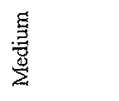 & 营 \\
\hline 泀 & & 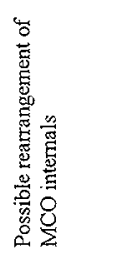 & 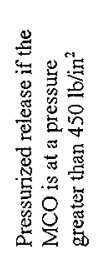 & & & 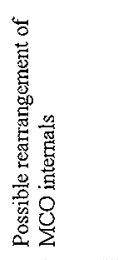 & 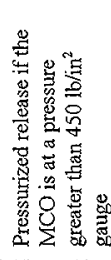 \\
\hline 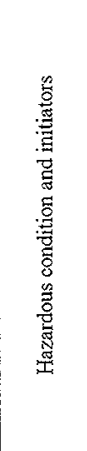 & 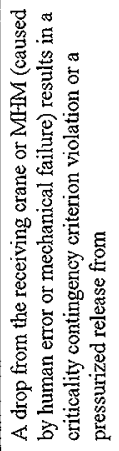 & 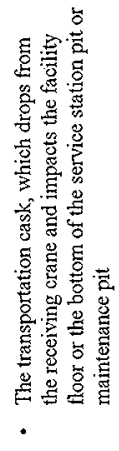 & 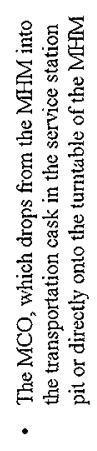 & 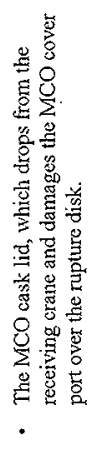 & 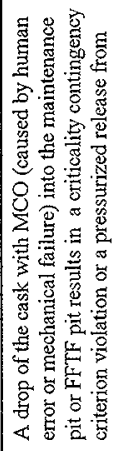 & 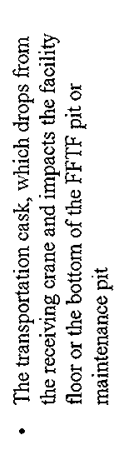 & 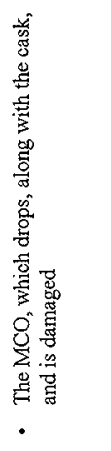 \\
\hline 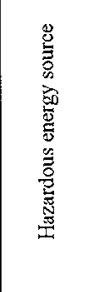 & 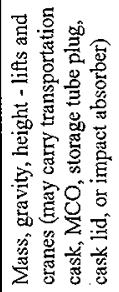 & & & & 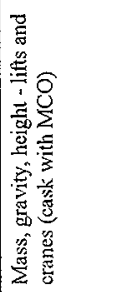 & & \\
\hline 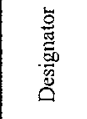 & 离 & & & & 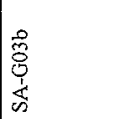 & & \\
\hline
\end{tabular}


HNF-SD-SNF-HIE-001 REV 1

\begin{tabular}{|c|c|c|c|c|c|c|c|c|c|c|}
\hline 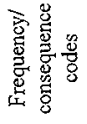 & & 怘 & $\underset{2}{\mathscr{C}}$ & 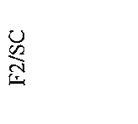 & 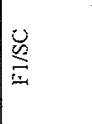 & 它 & 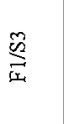 & & 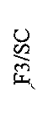 & $\underset{\mathrm{O}}{\mathrm{O}}$ \\
\hline 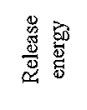 & & 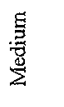 & 急 & 预 & 空 & 焉 & $\stackrel{3}{9}$ & & 产 & 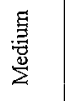 \\
\hline 占 & & 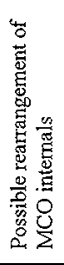 & 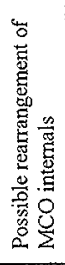 & 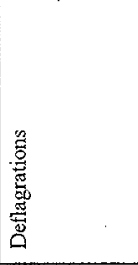 & 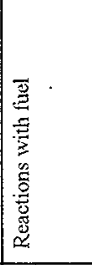 & 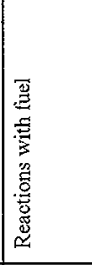 & 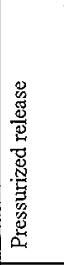 & & 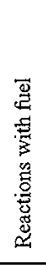 & 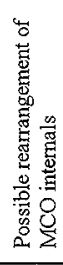 \\
\hline 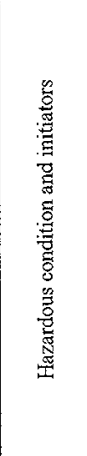 & 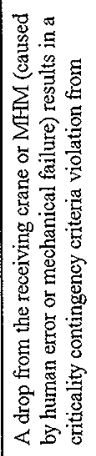 & 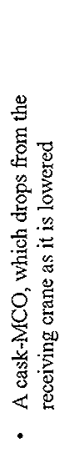 & 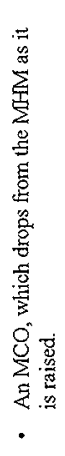 & 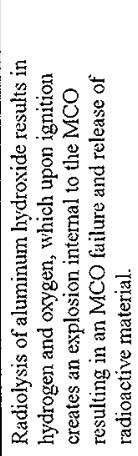 & 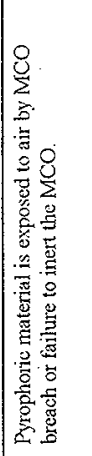 & 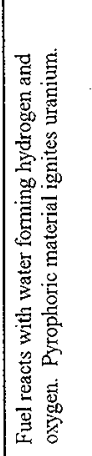 & 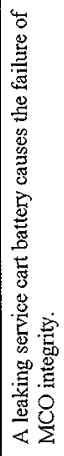 & 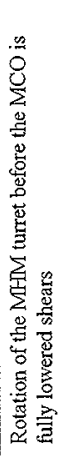 & 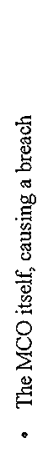 & 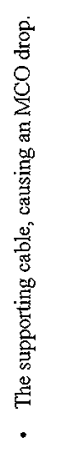 \\
\hline 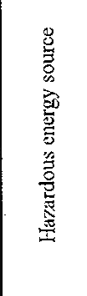 & 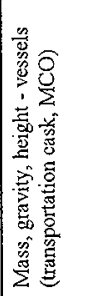 & & & 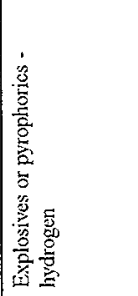 & 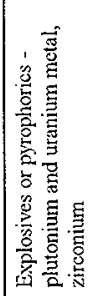 & 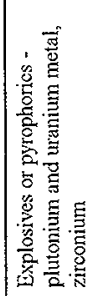 & 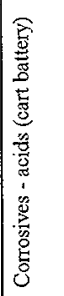 & 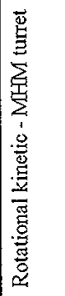 & & \\
\hline 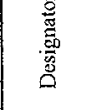 & $\stackrel{m}{0}$ & & & 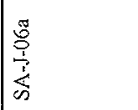 & $\frac{5}{\frac{3}{3}}$ & $\frac{0}{0}$ & $\frac{0}{0}$ & 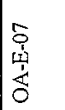 & & \\
\hline
\end{tabular}


HNF-SD-SNF-HIE-001 REV 1

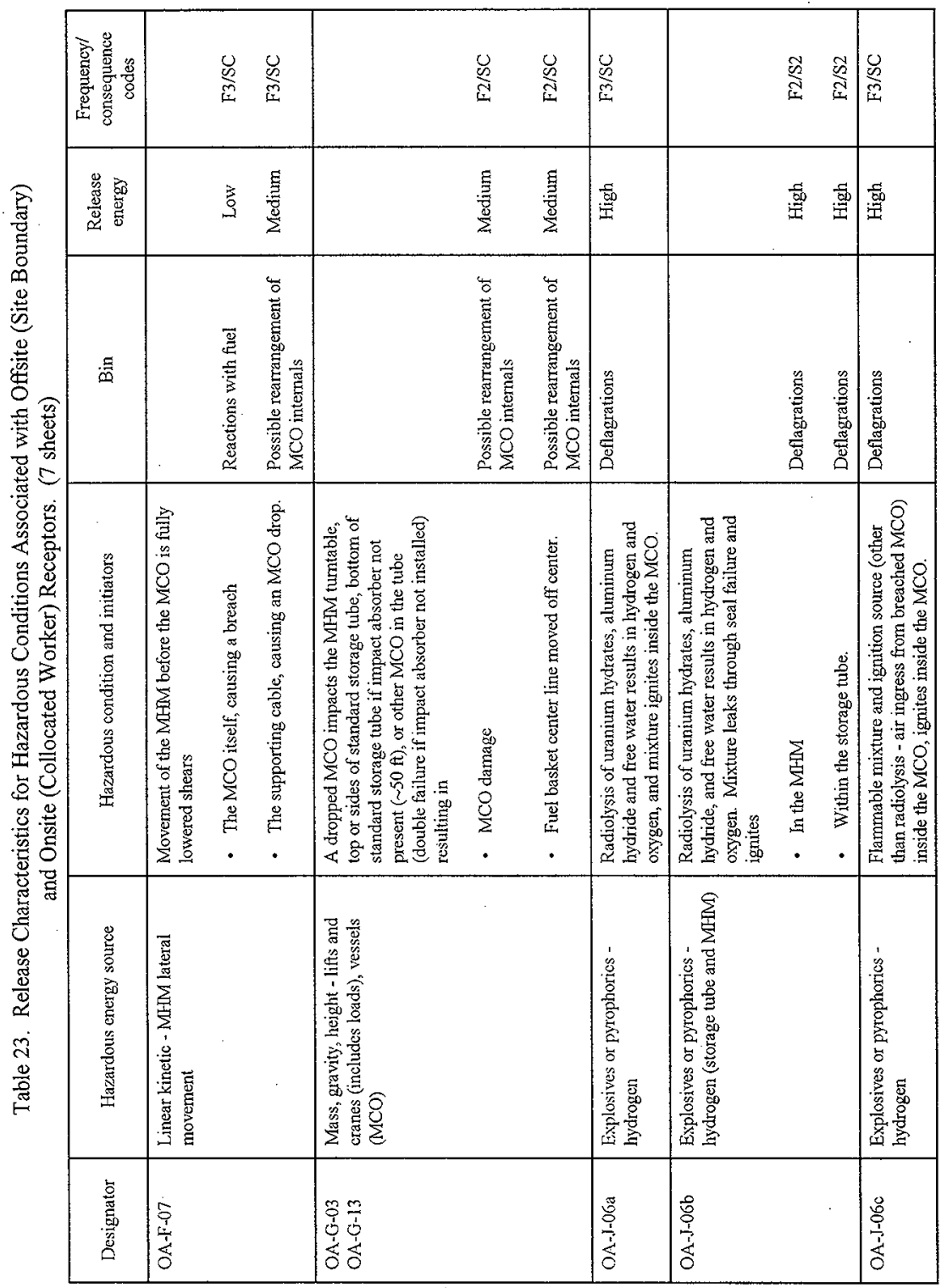


HNF-SD-SNF-HIE-001 REV 1

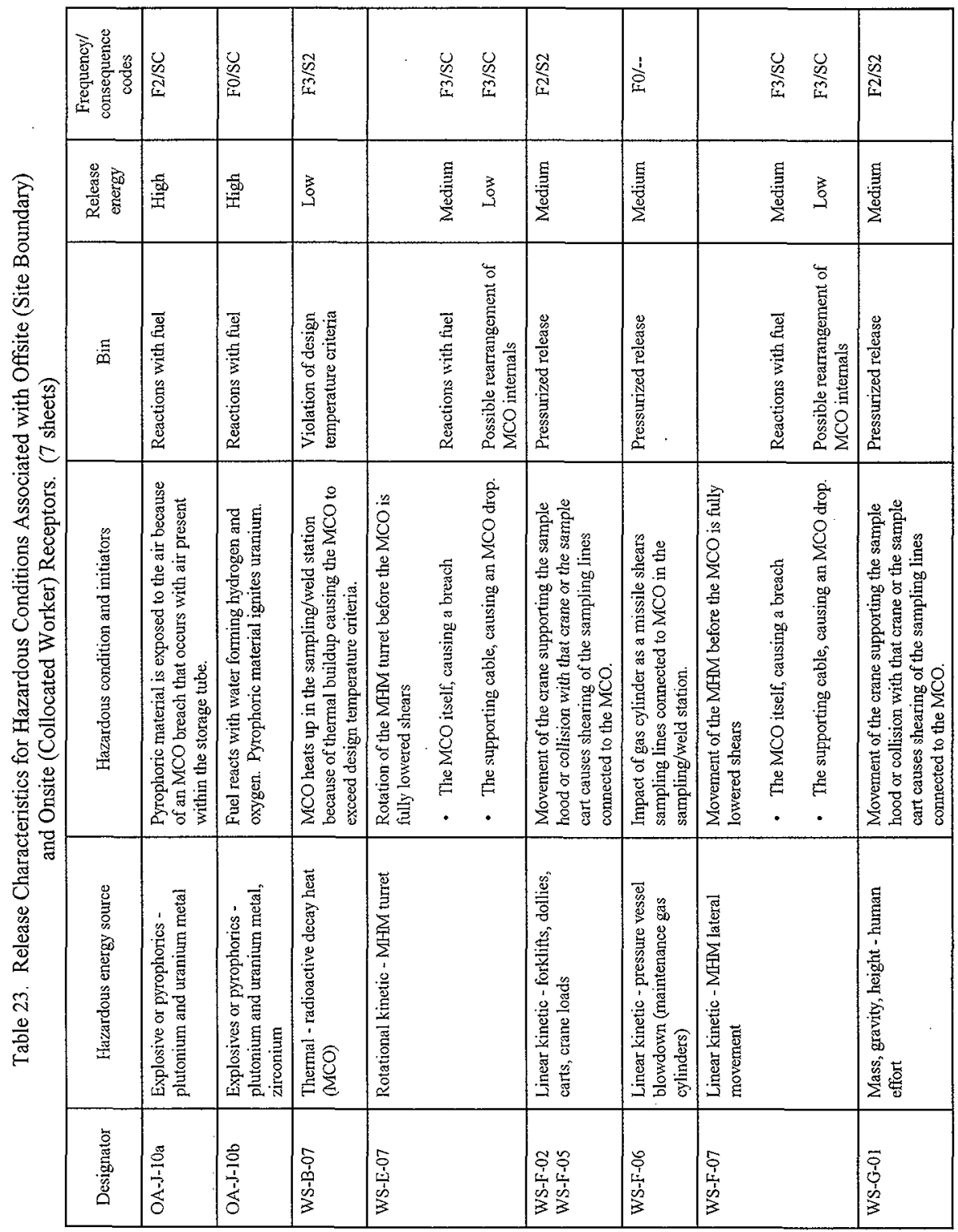


HNF-SD-SNF-HIE-001 REV 1

\begin{tabular}{|c|c|c|c|c|c|c|c|c|c|c|c|}
\hline 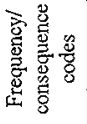 & & 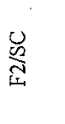 & $\frac{\bigcup}{0}$ & तี & कूष & $\frac{\widetilde{d}}{\mathrm{~N}}$ & 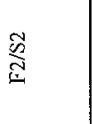 & $\frac{m}{\tilde{m}} \quad \frac{m}{\tilde{m}}$ & $\frac{0}{2}$ & $\frac{0}{2}$ & 范 \\
\hline 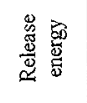 & & 鵷 & 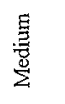 & 莺 & $\frac{\Xi}{g}$ & 雍 & 曋 & 急 步 & 点 & 要 & 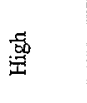 \\
\hline 蛋 & & 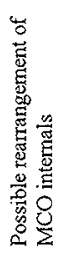 & 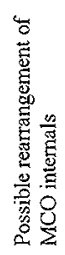 & 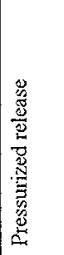 & 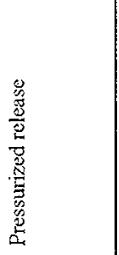 & 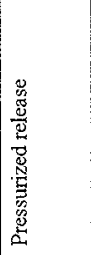 & 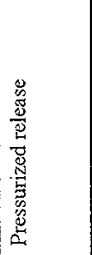 & 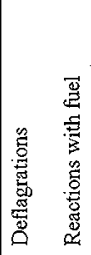 & 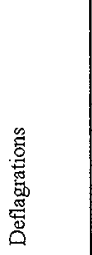 & 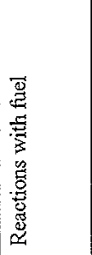 & 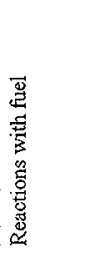 \\
\hline 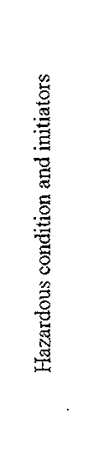 & 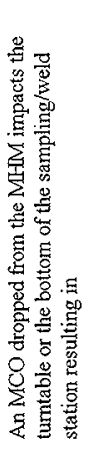 & 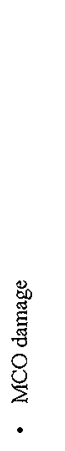 & 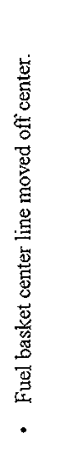 & 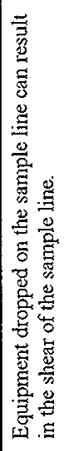 & 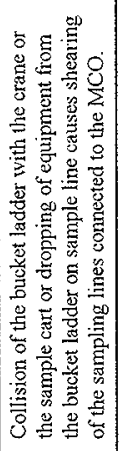 & 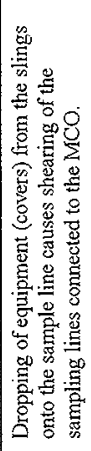 & 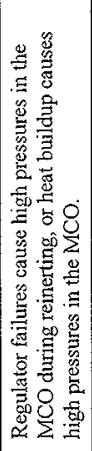 & 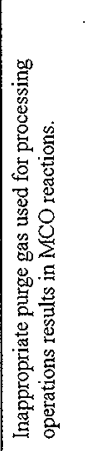 & 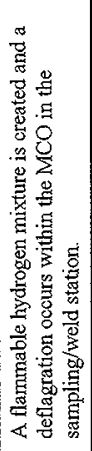 & 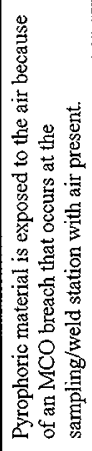 & 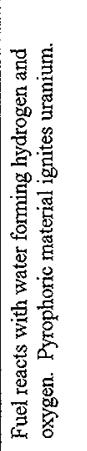 \\
\hline 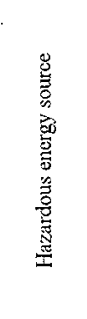 & 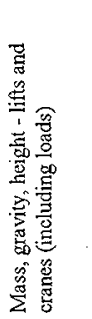 & & & 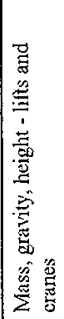 & 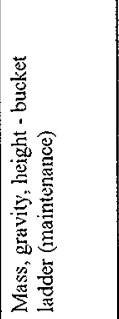 & 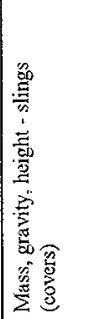 & 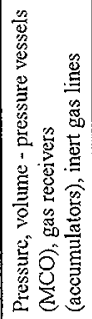 & 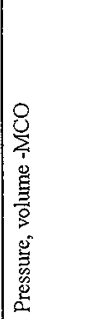 & 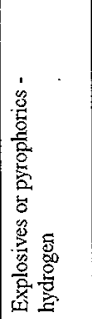 & 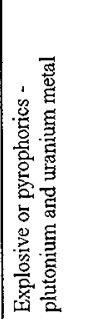 & 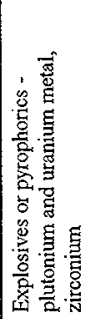 \\
\hline 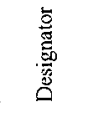 & 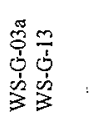 & & & $\begin{array}{l}0 \\
0 \\
0 \\
0 \\
1 \\
\vdots \\
3\end{array}$ & $\begin{array}{l}0 \\
0 \\
0 \\
\vdots \\
0 \\
3 \\
3\end{array}$ & $\begin{array}{l}8 \\
\vdots \\
0 \\
j \\
0 \\
3\end{array}$ & 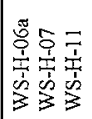 & $\begin{array}{l}0 \\
0 \\
\frac{1}{1} \\
\dot{1} \\
3 \\
3\end{array}$ & $\frac{8}{9}$ & $\begin{array}{l}\frac{5}{0} \\
\frac{1}{3} \\
\frac{1}{3} \\
3\end{array}$ & $\frac{8}{\frac{6}{6}}$ \\
\hline
\end{tabular}


HNF-SD-SNF-HIE-001 REV 1

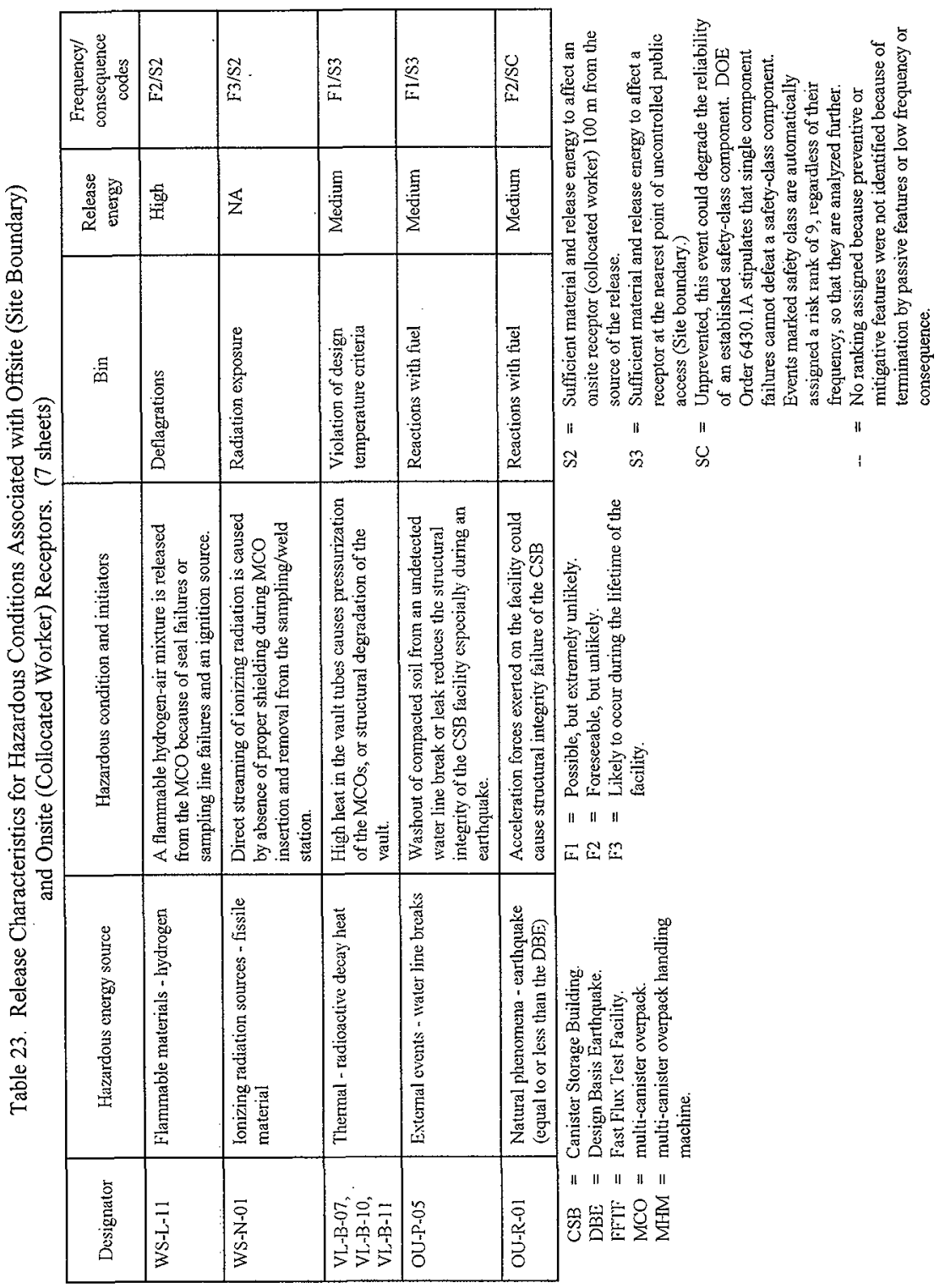


Table 24. Binned Listing of Candidate Accidents. (2 sheets)

\begin{tabular}{|c|c|c|c|}
\hline Candidate accident & Risk ranking & $\begin{array}{l}\text { Release or change } \\
\text { energy }\end{array}$ & Reference designator \\
\hline \multicolumn{4}{|c|}{ Rearrangenent of MCO internals (Section A3,4.2.1) } \\
\hline $\begin{array}{l}\text { Possible rearrangement of } \mathrm{MCO} \\
\text { internals due to a drop or shear }\end{array}$ & $\begin{array}{l}9 \\
9 \\
9 \\
9 \\
9 \\
9 \\
9 \\
9 \\
9 \\
9 \\
9 \\
6\end{array}$ & $\begin{array}{l}\text { Medium } \\
\text { Medium }^{b} \\
\text { Medium }^{b} \\
\text { Low }^{b} \\
\text { Low }^{b} \\
\text { Low }^{b} \\
\text { Low }^{b} \\
\text { Medium } \\
\text { Mediumb } \\
\text { Medium } \\
\text { Medium } \\
\text { Medium }\end{array}$ & $\begin{array}{l}\text { OA-E-07 } \\
\text { OA-F-07 } \\
\text { OA-G-03,-13 } \\
\text { SA-E-07 } \\
\text { SA-F-07b } \\
\text { WS-E-07 } \\
\text { WS-F-07 } \\
\text { WS-G-03a, -13 } \\
\text { SA-G-03a, -03b,-13 } \\
\text { TV-G-13 } \\
\text { OU-R-01 } \\
\text { OU-P-05 }\end{array}$ \\
\hline $\begin{array}{l}\text { Possible rearrangement of } \mathrm{MCO} \\
\text { internals due to collision }\end{array}$ & 8 & Medium & SA-F-05 \\
\hline $\begin{array}{l}\text { Possible rearrangement of } \mathrm{MCO} \\
\text { internals due to impact from gas } \\
\text { cylinder }\end{array}$ & 5 & Medium & $\begin{array}{l}\text { TV }-F-06 \\
\text { SA-F-06 } \\
\text { WS-F-06 }\end{array}$ \\
\hline \multicolumn{4}{|c|}{ Gaseous release from the MCO (Section A3.4.2.2) } \\
\hline Pressurized release from $\mathrm{MCO}^{\mathrm{a}}$ & $\begin{array}{l}9 \\
6 \\
5 \\
5 \\
5 \\
5 \\
5 \\
5 \\
5\end{array}$ & $\begin{array}{l}\text { Medium } \\
\text { Low } \\
\text { High } \\
\text { Medium } \\
\text { Medium } \\
\text { Medium } \\
\text { Medium } \\
\text { Medium } \\
\text { Medium }\end{array}$ & $\begin{array}{l}\text { SA-G-03a } \\
\text { OA-D-01 } \\
\text { WS-H-06a, -07,-11 } \\
\text { WS-F-02,-05 } \\
\text { WS-F-06 } \\
\text { WS-G-01 } \\
\text { WS-G-03b } \\
\text { WS-G-04 } \\
\text { WS-G-06 }\end{array}$ \\
\hline Pressurized release from cask-MCO & 9 & Low & $\mathrm{TV}-\mathrm{G}-13$ \\
\hline \multicolumn{4}{|c|}{$\mathrm{MCO}$ internal hydrogen explosion (Section $\mathrm{A3}$.4.2.3) } \\
\hline Hydrogen deflagration ${ }^{a}$ & $\begin{array}{l}9 \\
9 \\
9 \\
9\end{array}$ & $\begin{array}{l}\text { High } \\
\text { High } \\
\text { High } \\
\text { High }\end{array}$ & $\begin{array}{l}\text { WS-H-06b } \\
\text { WS-J-06 } \\
\text { OA-J-06a, -06c } \\
\text { SA-J-06a }\end{array}$ \\
\hline \multicolumn{4}{|c|}{ MCO external hydrogen explosion (Section A3.4.2.4) } \\
\hline External deflagration ${ }^{3}$ & $\begin{array}{l}5 \\
9\end{array}$ & $\begin{array}{l}\text { High } \\
\text { High }\end{array}$ & $\begin{array}{l}\text { WS-L-11 } \\
\text { WS-Y-06b }\end{array}$ \\
\hline
\end{tabular}


Table 24. Binned Listing of Candidate Accidents. (2 sheets)

\begin{tabular}{|c|c|c|c|}
\hline Candidate accident & Risk ranking & $\begin{array}{l}\text { Release or change } \\
\text { energy }\end{array}$ & Reference designator \\
\hline \multicolumn{4}{|c|}{ Thermal runaway fucl reactions inside the $M C O$ (Section $A 3,4,2.5$ ) } \\
\hline Runaway reaction ${ }^{a}$ & 9 & High & WS-H-O6b \\
\hline Fuel reaction with air & $\begin{array}{l}9 \\
9 \\
9\end{array}$ & $\begin{array}{l}\text { High } \\
\text { High } \\
\text { High }\end{array}$ & $\begin{array}{l}\text { SA-J-10a } \\
\text { OA-J-10a } \\
\text { WS-J-10a }\end{array}$ \\
\hline Fuel reaction with water & $\begin{array}{l}9 \\
9 \\
9\end{array}$ & $\begin{array}{l}\text { High } \\
\text { High } \\
\text { High }\end{array}$ & $\begin{array}{l}\text { SA-J-10b } \\
\text { OA-J-10b } \\
\text { WS-J-10b }\end{array}$ \\
\hline Fuel exposed to air & $\begin{array}{l}9 \\
9 \\
9 \\
9 \\
9 \\
9 \\
9\end{array}$ & $\begin{array}{l}\text { Medium } \\
\text { Medium } \\
\text { Low } \\
\text { Low } \\
\text { Low } \\
\text { Low } \\
\text { Low }\end{array}$ & $\begin{array}{l}\text { WS-E-07 } \\
\text { WS-F-07 } \\
\text { SA-E-07 } \\
\text { SA-F-07b } \\
\text { OA-E-07 } \\
\text { OA-F-07 } \\
\text { SA-F-05 }\end{array}$ \\
\hline \multicolumn{4}{|c|}{ Volations of design temperature criteria (Scetion A3.4.2.6) } \\
\hline $\begin{array}{l}\text { Violation of design temperature } \\
\text { criteria }^{2}\end{array}$ & $\begin{array}{l}7 \\
6\end{array}$ & $\begin{array}{l}\text { Low } \\
\text { Medium }\end{array}$ & $\begin{array}{l}\text { WS-B-07 } \\
\text { VL-B-07 }\end{array}$ \\
\hline
\end{tabular}

${ }^{a}$ Chosen as a representative and bounding accident for further accident analysis development.

'Energy was considered that could rearrange the internals of the MCO - falling onto the deck was viewed as higher energy than falling into the service or sample pit with impact absorbers present; falling into the tube with impact absorbers present was viewed as higher energy than falling into the service or the sample pit with an impact absorber present.

$\mathrm{MCO}=$ multi-canister overpack. 
Table 25. Three-by-Three Likelihood and Consequence Ranking Matrix.

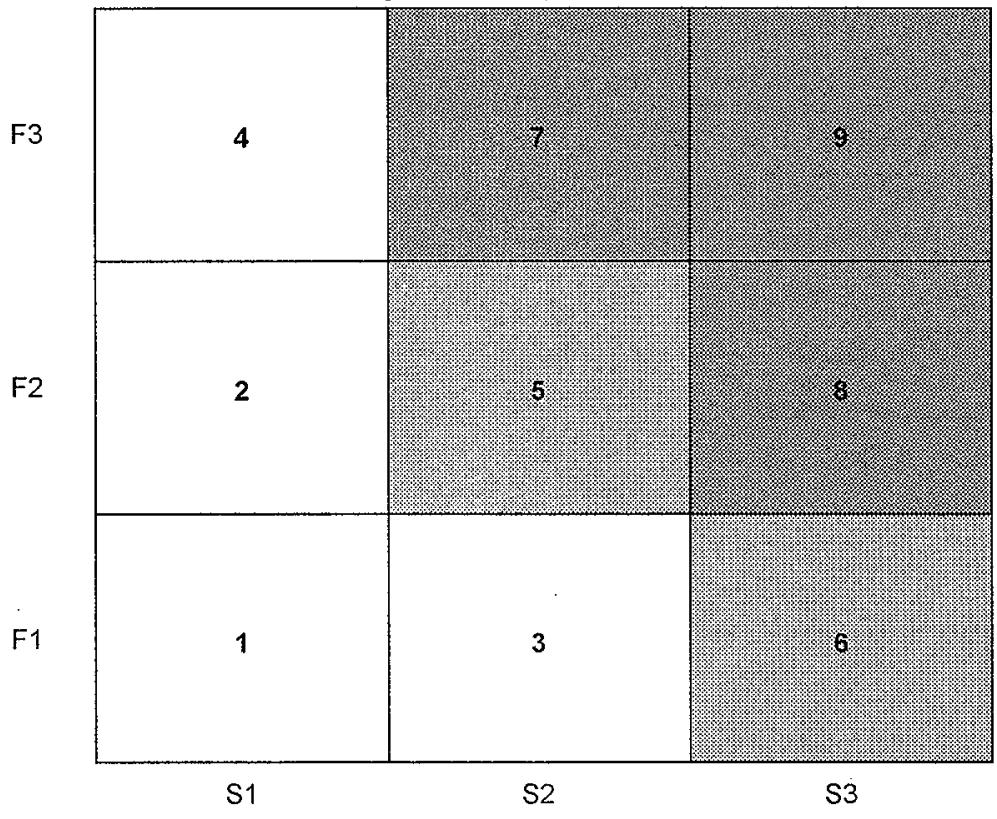

Combinations that identify situations of major concern

Combinations that identify situations of concern 

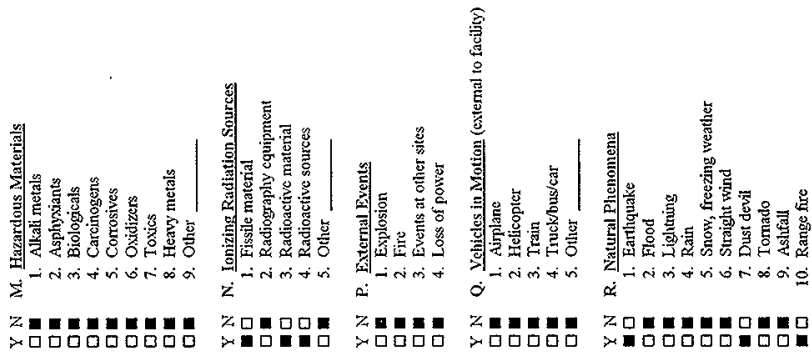

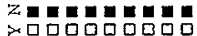

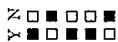

ำดㅁำ

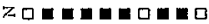

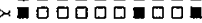

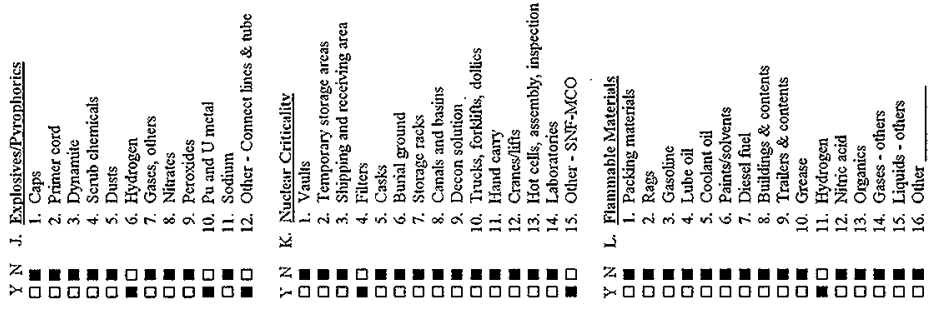

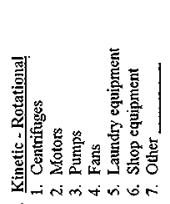

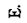

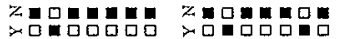
-

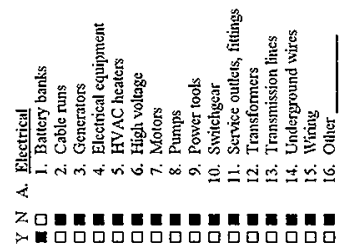

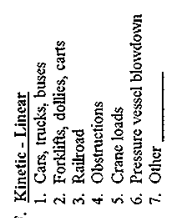

$$
\text { c }
$$

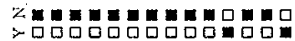

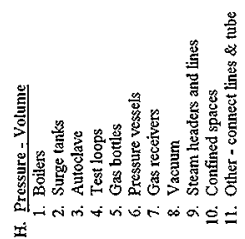

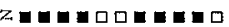
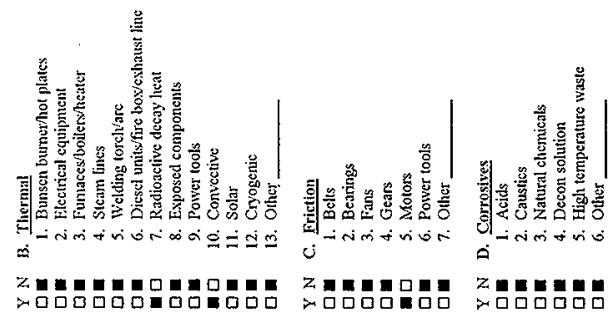
HNF-SD-SNF-HIE-001 REV 1

This page intentionally left blank. 


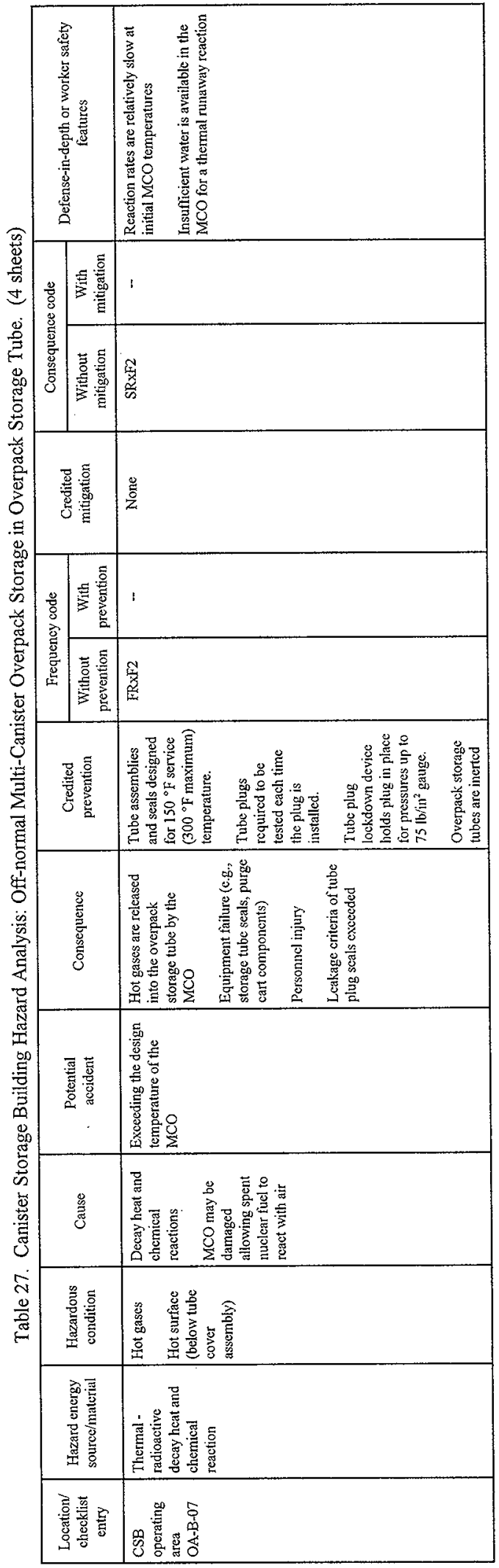




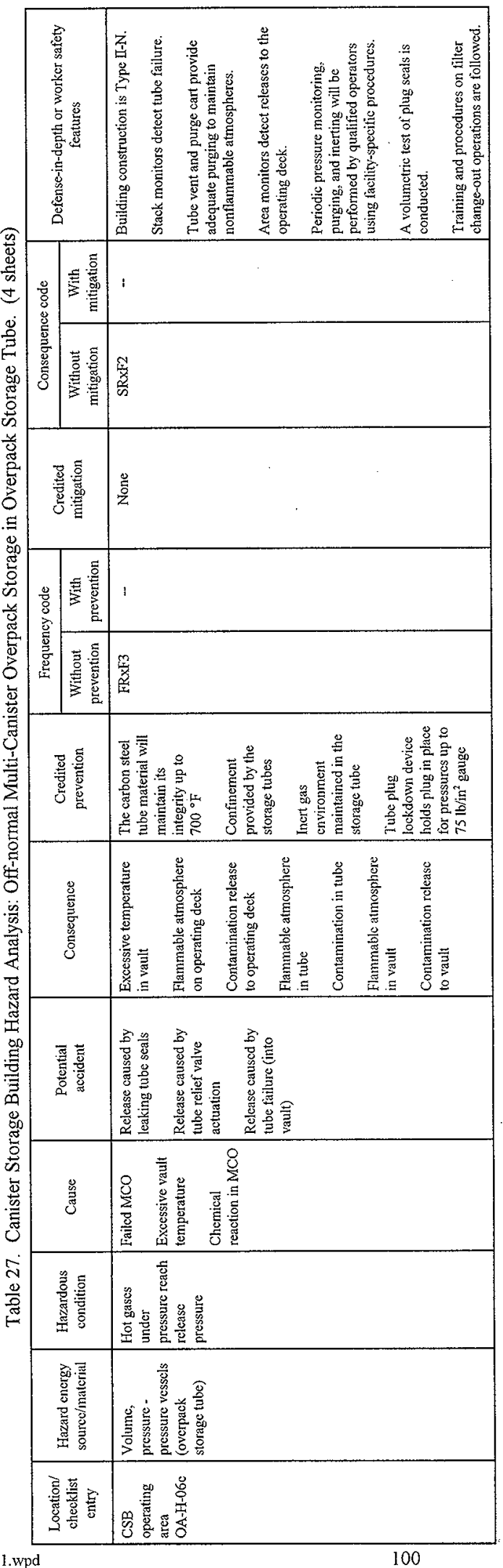


HNF-SD-SNF-HIE-001 REV 1

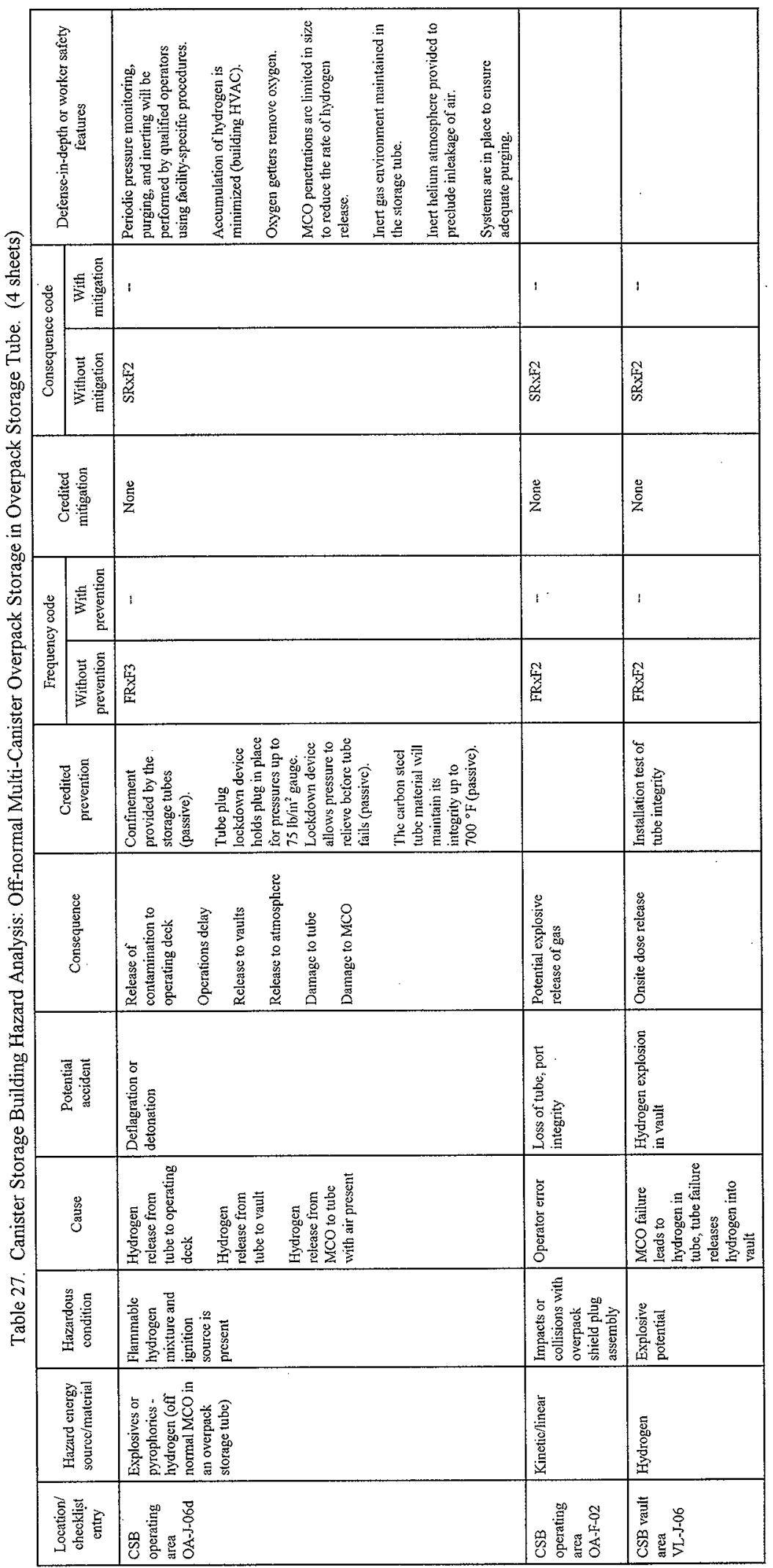


HNF-SD-SNF-HIE-001 REV 1

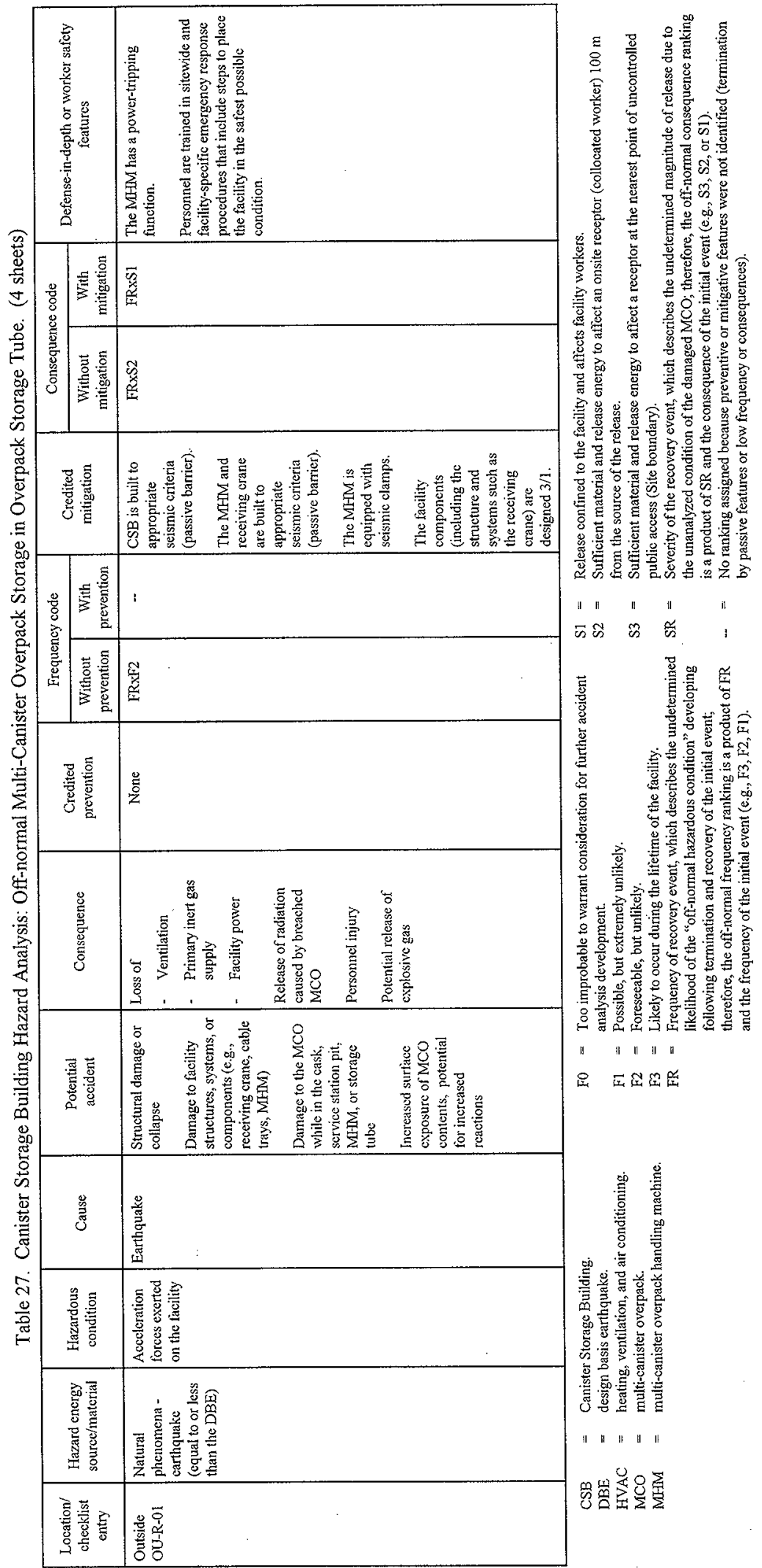


Table 28. Binned Listing of Candidate Accidents for Off-normal Multi-Canister Overpack Storage.

\begin{tabular}{|c|c|c|c|}
\hline Candidate accident & Risk ranking a & $\begin{array}{l}\text { Release or change } \\
\text { energy }\end{array}$ & $\begin{array}{l}\text { Reference } \\
\text { designator }\end{array}$ \\
\hline \multicolumn{4}{|c|}{ Gaseous releases and explosions from overpack storage tubes } \\
\hline Gaseous release in the overpack storage tube & $\begin{array}{l}\mathrm{FR} \times F 2 / \mathrm{SR} \times \mathrm{S} 2 \\
\mathrm{HR} \times \mathrm{F} 2 / \mathrm{SR} \times \mathrm{S} 2\end{array}$ & $\begin{array}{l}\text { Medium } \\
\text { low }\end{array}$ & $\begin{array}{l}\mathrm{OA}-\mathrm{B}-07 \\
\mathrm{OA}-\mathrm{F}-02\end{array}$ \\
\hline $\begin{array}{l}\text { Hydrogen explosions in the overpack storage } \\
\text { tube }\end{array}$ & $\begin{array}{l}\mathrm{FRxF}=3 / \mathrm{SR} \times \mathrm{S} 2 \\
\mathrm{FR} \times \mathrm{F} 2 / \mathrm{SR} \times \mathrm{S} 2 \\
\mathrm{FR} \times \mathrm{F} 2 / \mathrm{SR} \times \mathrm{S} 2 \\
\mathrm{FR} \times 2 / \mathrm{SR} \times \mathrm{S} 2\end{array}$ & $\begin{array}{l}\text { Medium } \\
\text { High } \\
\text { Medium } \\
\text { Low }\end{array}$ & $\begin{array}{l}\text { OA-J-06d } \\
\text { OU-R-01 } \\
\text { VL-J-06 } \\
\text { OA-F-02 }\end{array}$ \\
\hline $\begin{array}{l}\text { Gaseous release from the overpack storage tube } \\
\text { to the operating area }\end{array}$ & FrxF3/SRxS2 & Medium & $\mathrm{OA}-\mathrm{H}-06 \mathrm{c}$ \\
\hline
\end{tabular}

aFR Frequency of recovery event, which describes the undetermined likelihood of the "off-normal hazardous condition" developing following termination and recovery of the initial event; therefore, the off-normat frequency ranking is a product of FR and the frequency of the initial event (e.g., F3, F2, F1).

SR Severity of the recovery event, which describes the undetermined magnitude of release due to the unanalyzed condition of the damaged MCO; theretore, the oll-normal consequence ranking is a product of SR and the consequence of the initial event (e.g., $S 3, \$ 2$, or $\$ 1$ ). 
HNF-SD-SNF-HIE-001 REV 1

This page intentionally left blank. 
HNF-SD-SNF-HIE-001 REV 1

\section{ATTACHMENT A \\ CANISTER STORAGE BUILDING \\ HAZARD ANALYSIS \\ TEAM MEMBERS}


HNF-SD-SNF-HIE-001 REV I

This page intentionally left blank. 


\section{ATTACHMENT A}

\section{CANISTER STORAGE BUILDING \\ HAZARD ANALYSIS \\ TEAM MEMBERS}

The key members of the Canister Storage Building (CSB) hazard analysis team brought to the study the following experience.

\section{Walter Alaconis}

B.S., General Science, The University of the State of New York, Albany. Nearly 27 years of diversified nuclear safety and operations experience in the military, commercial, and U.S. Department of Energy (DOE) environments. Obtained registration with the National Registry of Radiation Protection Technologists in 1982. Over 16 years at the Hanford Site supporting major facility modifications and new facility design projects. Co-author of the Process Facility Modification Project Preliminary Safety Analysis Report. Managed the development of the Hanford Site Quality Training and Resource Center Root Cause Analysis Training Program and the Accident/Event Trending Program. Managed the Nuclear Engineering/Safety Data Management Unit for 4 years. Technical advisor to the Liquid Effluent Services Program at the Hanford Site and the Environmental Restoration Programs at the Hanford Site and DOEHeadquarters. Facilities supported at the Hanford Site include the tank farms (east), PUREX, B Plant, Plutonium Finishing Plant, Treated Effluent Disposal Facility, and Effluent Treatment Facility.

\section{Theodore Z. Anderson}

B.S., Mechanical Engineering. More than 24 years experience in facility operations, maintenance and quality assurance, and construction project quality assurance. Twenty years experience in existing nuclear facilities (Analytical Laboratory, fuel processing facilities) and new nuclear construction projects (tank farms, pipelines, spent nuclear fuel handling). Present position includes project construction quality assurance support to CSB multi-canister overpack handling machine and receiving crane procurements.

\section{Ralph D. Crowe}

M.S., Nuclear Engineering and Engineering Management. Over 20 years experience in the nuclear industry performing calculations using multidimensional, time-dependent, neutron kinetics and thermal hydraulic codes. Six years experience performing safety analysis within the DOE environment for a number of facilities, including high-level waste tanks, Plutonium Finishing Plant, and spent fuel storage. 


\section{William A. Frier}

Over 21 years experience in nuclear industry. Managed safety-class project upgrades, $\mathrm{K}$ Basin seismic analyses, Basins Life Extension Program, and K Basins Roof Repair Program. Current design authority for $\mathrm{K}$ Basins structures and cranes and hoists.

\section{Manuel Guzman}

Fourteen years experience in the nuclear industry. Started in the bargaining unit as a Chief Reactor Fuel operator at the $\mathrm{N}$ Reactor, worked up through the management chain as a shift supervisor responsible for around-the-clock operations loading, packaging, and shipping 120 metric tons of irradiated spent nuclear fuel per month. Transferred to the $\mathrm{K}$ Basins in a supervisory role and also served as a shift manager. Presently assigned to the CSB as a plant engineer in the Facility Startup and Operations organization providing input to the CSB design.

\section{Danny R. Henry}

B.A. degree. Over 19 years of varied Hanford Site work experience as an individual contributor and manager, involving both operational support and oversight functions at reactor, nuclear, and nonnuclear facilities. Experience includes positions and training as a firefighter and emergency medical technician, certified control room reactor operator, nuclear process standards engineer, senior reactor outage planner, outage manager at an operating production reactor facility, a Principal Nuciear Safety Department. Responsible for the development and implementation of both a company-wide Occupational Safety and Health Administration (OSHA) compliance inspection program and an OSHA-based baseline hazard assessment program for Westinghouse Hanford Company, as well as the coordination of the company programs and activities for gaining acceptance into the DOE/OSHA Voluntary Protection Program. Served as the company contact for the Westinghouse government-owned, contractor-operated subcommittee on industrial safety and as the primary point of contact for discipline-related interface meetings with the DOE counterpart. Designated contact for external audits, assessments, and reviews involving the safety programs. Served on special safety task forces, including the review and selection of contracted safety and health services.

\section{Taber G. Hersum}

M.S., Nuclear Engineering, registered professional engineer (California). Twenty-six years experience in nuclear safety, reactor physics, fuel loading and power ascension testing, operations, and technical and quality consulting for nuclear power plants, nuclear fuel, and nuclear waste in the United States, Europe, and Japan. Currently a member of the Fluor Daniel Hanford, Inc., Nuclear Safety organization providing oversight review of Project Hanford Management Contractor safety basis documents requiring DOE approval. Previously certified as a senior reactor operator and ANSI 45.2.6 Level III senior reactor operator test engineer. 


\section{Maurice J. Higuera}

B.S., Mechanical Engineering; M.S., Program Engineering Management; B.S., Human Biology/Organic Chemistry. Twenty-plus years experience in U.S. Nuclear Regulatory Commission and DOE regulatory environment. Areas of expertise include nuclear project management and engineering; environmental, safety, and industrial health; licensing and regulatory compliance; system process, operations hazard, and operability studies; outage planning and scheduling; and startup and testing and commissioning. Currently lead independent safety engineer and lead safety analysis report chapter author, engineering and coordinating input to the safety analysis report for the CSB spent nuclear fuel storage mission.

\section{Larry D. Kessie, AIA}

B.S., Architectural Studies, B. of Architecture, Registered Architect. Fifteen years experience in architectural design, design management, cost account management, project management, and construction administration in both the private and government sectors. Positions have ranged from that of a project architect and design office architect to temporary facility and site infrastructure design authority. Currently serving as consulting architect/design authority for site infrastructure and temporary facilities for Project W-379, Spent Nuclear Fuel CSB.

\section{Steve D, Kopelic}

B.S., Mechanical Engineering. Twenty years experience related to commercial pressurized water reactor electric generation stations. Experience includes reactor core design and testing, primary and secondary cooling systems accident analysis, preparation and defense of plant licensing documentation, probabilistic risk assessment, and implementation of design modifications on operating plants. Currently performing safety analyses and preparing safety analysis reports for the Spent Nuclear Fuel Project.

\section{Dwight E. Krahn}

B.S., General Engineering, field of specialty in Operations Research. Eight years experience in engineering and safety analysis activities. Training includes safety analysis development, root cause analysis, and risk assessment. Most recent work has been in the area of technical safety requirements for the Waste Encapsulation and Storage Facility and the tank farms. 


\section{Maryanne Kummerer}

M.S., Mechanical Engineering. Fifteen years experience in engineering analysis in the area of heat transfer, multiphase fluid flow, thermodynamics, and chemical phenomena. Nine years performing calculations in support of safety analysis for various Hanford Site facilities and providing accident analysis contributions to safety documentation, including safety assessments and safety analysis reports.

\section{Barclay S. Lew}

Twenty-five years of nuclear industry experience in safety analysis, engineering applications, and licensing and regulatory affairs for U.S. and international electric utilities, DOE, and international laboratory facilities and universities. Senior manager at Pacific Gas \& Electric Company (PG\&E) in regulatory and safety analysis of PG\&E's pressurized water reactor and boiling water reactor facilities. As a consultant, performed risk-based prioritization studies for several DOE facilities to assist in the segregation and focus of issues important to public safety, site worker safety, environmental, and mission. Prepared and evaluated safety analyses and risk analyses related to final safety analysis report limiting conditions for operation requirements, hazard evaluation, and operational conditions, including fire detection and protection, and risk/detection/reliability studies. Responsible for the development and implementation of light water reactor nuclear analytical methods and analysis for applications to encore physics, criticality analysis, fuel management, thermal-hydraulics, fuel element performance, transient safety, and accident analysis for pressurized water reactors for several U.S. utilities. Recently published numerical simulation of multiphase heat transfer and fluid flow and has numerous past publications in safety analysis and regulatory applications.

\section{James Mathews}

Over 25 years of experience in the chemical and nuclear industry. Sixteen years experience at the Hanford Site working in the Operations, Fuel Handling and Waste and Radiological Control organizations. Provided management direction and oversight of daily and special operations. Played an integral role during the K East fuel segregation program and the PUREX fuel shipping programs and later transitioned to managing decontamination and waste control activities after the shutdown of $\mathrm{N}$ Reactor. The past 2 years he has been the manager of Project Operations for the Spent Nuclear Fuel Project with responsibility for reviewing all designs and safety documentation to ensure that new facilities and components can be operated and maintained safely and efficiently. Certified as a nuclear material custodian, hazardous material/waste shipper, hazardous waste coordinator, and an unreviewed safety question evaluator for the Spent Nuclear Fuel Project. 


\section{Robert E. Piippo}

B.S., Industrial Technology. Eighteen years experience in program management, planning, operations, and engineering within the nuclear and aerospace industry. Positions have included program manager/chief engineer, nuclear safety assurance principal engineer, lead aerospace systems engineer, advanced engineering manager for $\mathrm{N}$ Reactor fuels manufacturing, manager for fuels manufacturing, and lead aerospace manufacturing engineer. Performed nuclear safety oversight as a member of the operational readiness review boards for the startup and operation of the $\mathrm{UO}_{3}$ calcination processing facility, $\mathrm{N}$ Reactor irradiated fuel element encapsulation, and grouting of low-level liquid waste. Participated in nuclear safety appraisals for PUREX, B Plant/Waste Encapsulation and Storage Facility, and fuels manufacturing. Currently performing nuclear safety on the Spent Nuclear Fuel Program.

\section{Thomas B. Powers}

B.S., Chemical Engineering. Over 23 years of experience, including 15 years of experience in systems safety analysis and risk assessment for both nuclear and nonnuclear facilities and operations and 8 years of experience in environmental analysis, engineering design, and testing of nuclear and nonnuclear systems. Worked on N Reactor and Fast Flux Test Facility Level I probabilistic risk assessments and performed numerous safety analyses using preliminary hazard analyses, failure modes and effects analyses, fault trees, and event tree methods.

\section{David L. Scott}

M.S., Chemical Engineering. Twenty-six years of professional experience in safety analysis, process engineering, project engineering and development work related to the nuclear and petrochemical industries. Over 5 years of experience at the Hanford Site working in safety analysis and risk assessment. Work in other areas includes fluid flow analysis, pump and piping system design, uranium processing, $N$ Reactor modification engineering, $N$ Reactor nuclear fuel manufacturing, and organic chemical production.

\section{W. Todd Watson}

M.S., Physics (Experimental Nuclear Physics), University of Illinois at Urbana-Champaign. Six years experience in the development of nuclear measurement systems, instrumentation, and computer data acquisition and analysis systems. Experience performing computer modeling of radiation transport, including nuclear criticality analyses, dose rate predictions, and nuclear characterization instrumentation response modeling. One year of experience performing criticality safety evaluations for the Hanford Plutonium Finishing Plant and evaluating the adequacy of criticality accident alarm systems for the Rocky Flats Environmental Technology Site. One year of experience performing safety analysis in support of Hanford Site tank farms and the Spent Nuclear Fuel Program. 
Others who participated in the hazards analysis meetings and provided information to assist the process included

- Jeff Parker, Ares Corporation, facilitator for the sessions

- Richard Hulskamp, multi-canister overpack and receiving crane design information. 